\title{
Height Zeta Functions of Toric Bundles over Flag Varieties
}

\author{
Matthias Strauch and Yuri Tschinkel
}




\section{Contents}

1 Introduction $\quad 1$

2 Generalized flag varieties $\quad 11$

3 Toric varieties $\quad 13$

4 Twisted products $\quad 21$

5 Meromorphic continuation $\quad 27$

$6 \quad$ Non-vanishing of asymptotic constants 36

$\begin{array}{lll}7 & \text { Technical theorems } & 42\end{array}$

8 Some statements on Eisenstein series $\quad 56$

$\begin{array}{lll}9 & \text { Examples } & 67\end{array}$

\section{Introduction}

1.1 The basic problem. This paper is concerned with the study of rational points on certain projective varieties over number fields. These varieties are fiber bundles over generalized flag varieties and the fibers are equivariant compactifications of algebraic tori. Before giving a detailed description we will explain the basic problem in general and elementary terms.

Suppose we are given a finite set of homogeneous polynomials $\left\{P_{i}\right\}$ in $n+1$ variables with integer coefficients. Consider the system of equations

(*) for all $i: P_{i}\left(x_{0}, \ldots, x_{n}\right)=0$.

We are interested in the set of solutions of this system of equations having integer coordinates. For any simultaneous solution $x \in \mathbf{Z}^{n+1}$ and any $t \in \mathbf{Z}$ we get another solution, 
namely $t \cdot x$. Therefore, we are led to look for those $x=\left(x_{0}, \ldots, x_{n}\right) \neq 0$ for which $\operatorname{gcd}\left(x_{0}, \ldots, x_{n}\right)=1$. These $x$ are called primitive.

Obviously, for any given real number $H$ there are only finitely many primitive solutions $x$ of $(*)$ for which the height

$$
H(x):=\max \left\{\left|x_{0}\right|, \ldots,\left|x_{n}\right|\right\}
$$

does not exceed $H$. Provided that there are infinitely many primitive solutions it makes sense to study the number $N(H)$ of such $x$ for which $H(x) \leq H$ as a function of $H$. Now the basic problem is to understand the asymptotic behavior of this counting function as $H$ tends to infinity.

We can rephrase what has been said so far as follows. Let $X$ be a projective variety over $\mathbf{Q}$ and let $f: X \hookrightarrow \mathbf{P}^{n}$ be an embedding (over $\mathbf{Q}$ ) of $X$ into some projective space $\mathbf{P}^{n}$. Then the image of $X$ under $f$ may be described by a system of homogeneous polynomial equations with integer coefficients like $(*)$.

Any Q-rational point $x$ on $X$ gives a point in $\mathbf{P}^{n}(\mathbf{Q})$ which may be represented as an $(n+1)$-tuple $\left(x_{0}, \ldots, x_{n}\right)$ of integers with greatest common divisor equal to one, i.e., as a primitive solution to $(*)$. Now we can count rational points on $X$ with respect to the height function induced by $f$.

The simplest example is just the projective space $\mathbf{P}^{n}$ itself (over $\mathbf{Q}$ ). Here we may take $f$ to be the identity. In this case one has the task of counting primitive lattice points in $(n+1)$-dimensional cubes and thus $N(H)$ is asymptotically equal to

$$
\frac{2^{n}}{\zeta(n+1)} \cdot H^{n+1},
$$

where $\zeta$ denotes the Riemann zeta function. This is easily shown using the Moebius inversion formula. If we let $f: \mathbf{P}^{n} \rightarrow \mathbf{P}^{n^{\prime}}$ be the $d$-uple embedding, then the new height is the $d$-th power of the previous height and thus $N(H) \sim 2^{n} \zeta(n+1)^{-1} H^{(n+1) / d}$.

More generally, for an arbitrary number field $F$ and a point $x=\left[x_{0}: \ldots: x_{n}\right]$, in $\mathbf{P}^{n}(F)$ we may define the height of $x$ to be

$$
H(x):=\prod_{v} \max \left\{\left|x_{0}\right|_{v}, \ldots,\left|x_{n}\right|_{v}\right\}
$$

where $v$ runs over the set of all places of $F$ and $|\cdot|_{v}$ denotes the local norm corresponding to $v$ (suitably normalized). Then we can ask the same question as above for a projective variety $X$ over $F$ and an embedding $X \hookrightarrow \mathbf{P}^{n}$.

Eventually, we will define height functions intrinsically, by using metrized line bundles (cf. [31], [43]). In fact, there is no reason why one should prefer $\max \left\{\left|x_{i}\right|\right\}$ to $\left(\sum x_{i}^{2}\right)^{1 / 2}$ or 
$\sum\left|x_{i}\right|$. Instead of using the same height in all circumstances, it is desirable to allow the use of height functions associated to metrized line bundles which appear most naturally in the specific context under consideration.

1.2 Previous work connected with the subject. For some historical remarks concerning height functions and their first applications, for instance in the proof of the Mordell-Weil theorem, we recommend S. Lang's book Diophantine Geometry [21]. More recent results on diophantine problems can be found in the books [22],[23] by the same author. One of the first papers strictly devoted to counting points of bounded height is S. Schanuel's [38], where he considers the case of projective spaces over an arbitrary number field. This is less trivial than the example given above because one has to determine a fundamental domain modulo the action of the units (the proofs appeared in [39]). In 1965, Neron [29] gave asymptotics for the number of rational points of bounded height on abelian varieties. The classical circle method, now a whole field in itself, proves asymptotics on complete intersections of small degree. We refer to the papers by B. Birch, W. M. Schmidt and others (cf. [8], [40], [25]). In [41] W.M. Schmidt studied asymptotics for Grassmannians over Q.

E. Peyre and P. Salberger have independently developed a framework which allows to express the counting of rational points of bounded height on certain varieties in terms of counting of integral points in bounded domains on some canonical integral models of universal torsors over the original variety (cf. [32], [36]). Their theory can be viewed as a generalization of the counting of primitive solutions. In certain cases, like that of a projective space, complete intersections of small degree, blowups of projective spaces in projective spaces of higher dimension (cf. [31]), the equation $x y z=t^{3}$ (cf. [13], [9]), or split Fano toric varieties over $\mathbf{Q}$ (cf. [36]), one obtains asymptotics for rational points and alternative proofs of some special cases of varieties treated in this paper. To learn more about this point of view, we recommend the recent preprint [33]. We would like to point out that results about counting solutions of Pell's equation, as well as counting of integral points on some toric varieties (cf. the paper by B. Moroz [26] and references therein) are not contained in our paper.

Height zeta functions were introduced by S. Arakelov and G. Faltings, who proved asymptotics of effective zero cycles of fixed degree on curves of higher genus (cf.[1],[12]). This theory was taken up in [14] and developed in the papers [3], [4]. 
1.3 Examples. Now we give two concrete examples which will be studied in section 9 where we will use them to illustrate some of our constructions and results.

Let $n \geq 1$ be an integer and put

$$
X_{n}:=\left\{\left(\left[y_{0}: y_{1}: y_{2}\right],\left[x_{0}: x_{1}\right]\right) \in \mathbf{P}^{2} \times \mathbf{P}^{1} \mid y_{0} x_{1}^{n}-y_{1} x_{0}^{n}=0\right\} .
$$

This is a smooth projective surface over $\mathbf{Q}$, sometimes called a Hirzebruch surface. Consider the embedding of $X_{n}$ into $\mathbf{P}^{5}$ induced by the Segre embedding

$$
\left(\left[y_{0}: y_{1}: y_{2}\right],\left[x_{0}: x_{1}\right]\right) \mapsto\left[y_{0} x_{0}: y_{1} x_{0}: y_{2} x_{0}: y_{0} x_{1}: y_{1} x_{1}: y_{2} x_{1}\right] .
$$

The height of a Q-rational point $x$ on $X_{n}$ with respect to this embedding is

$$
\begin{gathered}
\max \left\{\left|y_{0} x_{0}\right|,\left|y_{1} x_{0}\right|,\left|y_{2} x_{0}\right|,\left|y_{0} x_{1}\right|,\left|y_{1} x_{1}\right|,\left|y_{2} x_{1}\right|\right\} \\
=\max \left\{\left|x_{0}\right|,\left|x_{1}\right|\right\} \cdot \max \left\{\left|y_{0}\right|,\left|y_{1}\right|,\left|y_{2}\right|\right\} .
\end{gathered}
$$

Here $\left(y_{0}, y_{1}, y_{2}\right)$ and $\left(x_{0}, x_{1}\right)$ are primitive integral vectors such that $x$ is represented by $\left(\left[y_{0}: y_{1}: y_{2}\right],\left[x_{0}: x_{1}\right]\right)$. Denote by $N(H)$ the number of rational points on $X_{n}$ for which the height does not exceed $H$. Then

$$
N(H) \sim \frac{8 \zeta(n+1)+2 \zeta(n+2)}{\zeta(2) \cdot \zeta(n+2)} H^{2} .
$$

For this simple example this asymptotic can be calculated by hand because one can "see" all rational points and compute their heights. In general however, especially over an arbitrary number field, this may not be the right strategy. In section 9 we will show how our general approach works in this particular example.

For the second example, let $x_{0}, x_{i j}, x_{i j}^{\prime}, 1 \leq i<j \leq 4$, be homogeneous coordinates in $\mathbf{P}^{12}$ and let $w_{i j}, 1 \leq i<j \leq 4$, be the homogeneous coordinates in $\mathbf{P}^{5}$. Let $Y$ be the subvariety of $\mathbf{P}^{12} \times \mathbf{P}^{5}$, defined by the following equations:

$$
w_{12} w_{34}-w_{13} w_{24}+w_{14} w_{23}=0
$$

for all $1 \leq i<j \leq 4,1 \leq k<l \leq 4: \quad x_{i j} w_{k l}-x_{k l} w_{i j}=0 \quad x_{i j}^{\prime} w_{k l}^{2}-x_{k l}^{\prime} w_{i j}^{2}=0$.

We will study Q-rational points on $Y$ with respect to the height function

$$
\max \left\{\left|x_{0}\right|,\left(\sum x_{i j}^{2}\right)^{1 / 2}, \sum\left|x_{i j}^{\prime}\right|\right\}^{3} \cdot\left(\sum w_{i j}^{2}\right)^{1 / 2}
$$


(as before, $\left(x_{0}, x_{i j}, x_{i j}^{\prime}\right)$ and $\left(w_{i j}\right)$ are primitive integral vectors.)

At first sight this height looks somewhat strange. But, as we will explain later, it comes from a metrized line bundle on $Y$ which is very natural in the setting we are going to build up.

In this example we can observe a phenomenon which did not appear in the previous example. Consider the subvariety of $Y$ given by

$$
x_{i j}=0, x_{i j}^{\prime}=0 \quad(1 \leq i<j \leq 4) .
$$

Any point of this subvariety has the form $\left([1: 0: \ldots: 0],\left[w_{i j}\right]\right)$ and the height is just $\left(\sum w_{i j}\right)^{1 / 2}$ (with primitive $\left(w_{i j}\right)$ ). The number of such points of height less than $H$ grows like $c \cdot H^{4}$ (for some $c>0$ ). On the other hand, let $Y^{o}$ be the open subset consisting of those points where $x_{0} \neq 0$ and at least one $x_{i j} \neq 0$ and at least one of the $x_{i j}^{\prime} \neq 0$. Then the number of rational points on $Y^{o}$ for which the height is less than $H$ is asymptotically equal to

$$
\frac{4}{7} \pi^{2} \frac{\zeta(2)}{\zeta(3)^{2} \zeta(4)} H \cdot \log H
$$

Hence it should be clear that, in general, it is necessary to exclude some "degenerate" or accumulating subvarieties to get a reasonable result (cf. [24], [14], [31]). This example will be taken up again in section 9 .

1.4 To introduce more general concepts and notations, we let $X$ be a nonsingular projective algebraic variety over a number field $F$. Let $\mathcal{L}=\left(L,\left(\|\cdot\|_{v}\right)_{v}\right)$ be a metrized line bundle on $X$, i.e., a line bundle $L$ together with a family of $v$-adic metrics, where $v$ runs over the set $\operatorname{Val}(F)$ of places of $F$. Associated to $\mathcal{L}$ there is a height function

$$
H_{\mathcal{L}}: X(F) \rightarrow \mathbf{R}_{>0}
$$

on the set $X(F)$ of $F$-rational points of $X$ (cf. $[45,31]$ for the definitions of $v$-adic metric, metrized line bundle and height function). For appropriate subvarieties $U \subset X$ and line bundles $L$, we have

$$
N_{U}(\mathcal{L}, H):=\left\{x \in U(F) \mid H_{\mathcal{L}}(x) \leq H\right\}<\infty
$$

for all $H$ (e.g., this holds for any $U$ if $L$ is ample). We are interested in the asymptotic behavior of this counting function as $H \rightarrow \infty$. It is expected that the behavior of such asymptotics can be described in geometric terms $([2,14])$. 
Let

$$
\Lambda_{\text {eff }}(X):=\sum_{H^{0}(X, L) \neq 0} \mathbf{R}_{\geq 0}[L] \subset \operatorname{Pic}(X)_{\mathbf{R}}
$$

be the closed cone in $\operatorname{Pic}(X)_{\mathbf{R}}$ generated by the classes of effective divisors $([L]$ denotes the class of the line bundle $L$ in $\operatorname{Pic}(X))$. Let $L$ be a line bundle on $X$ such that $[L]$ lies in the interior of $\Lambda_{\text {eff }}(X)$. Define

$$
a(L):=\inf \left\{a \in \mathbf{R} \mid a[L]+\left[K_{X}\right] \in \Lambda_{\mathrm{eff}}(X)\right\},
$$

where $K_{X}$ denotes the canonical line bundle on $X$. Assume that $\Lambda_{\text {eff }}(X)$ is a finitely generated polyhedral cone. For $L$ as above, we let $b(L)$ be the codimension of the minimal face of $\Lambda_{\mathrm{eff}}(X)$ which contains $a(L)[L]+\left[K_{X}\right]$.

By a Tauberian theorem (cf. [11], Théorème III), the asymptotic behavior of $N_{U}(\mathcal{L}, H)$ can be determined if one has enough information about the height zeta function

$$
Z_{U}(\mathcal{L}, s):=\sum_{x \in U(F)} H_{\mathcal{L}}(x)^{-s}
$$

More precisely, suppose that $Z_{U}(\mathcal{L}, s)$ converges for $\operatorname{Re}(s) \gg 0$, that it has an abscissa of convergence $a>0$ and that it can be continued meromorphically to a halfspace beyond the abscissa of convergence. Suppose further that there is a pole of order $b$ at $s=a$ and that there are no other poles in this halfspace. Then

$$
N_{U}(\mathcal{L}, H)=c H^{a}(\log H)^{b-1}(1+o(1))
$$

for $H \rightarrow \infty$ and

$$
c=\frac{1}{(b-1) ! a} \cdot \lim _{s \rightarrow a}(s-a)^{b} Z_{U}(\mathcal{L}, s) .
$$

It is conjectured that for appropriate $U$ and $\mathcal{L}$, one has $a=a(L)$ and $b=b(L)$ (cf. $[2,14])$. Moreover, there is a conjectural framework as to how to interpret the constant $c$ (cf. [31, 7]). There are examples which demonstrate that this geometric "prediction" of the asymptotic cannot hold in complete generality, even for smooth Fano varieties (cf. [6]). Our goal is to show that the conjectures do hold for a class of varieties closely related to linear algebraic groups. Our results are a natural extension of corresponding results for flag varieties (cf. [14]) and toric varieties (cf. [4, 5]). We proceed to describe the class of varieties under consideration. 
1.5 Let $G$ be a semi-simple simply connected split algebraic group over $F$ and $P \subset G$ an $F$-rational parabolic subgroup of $G$. Let $T$ be a split algebraic torus over $F$ and $X$ a projective nonsingular equivariant compactification of $T$. A homomorphism $\eta: P \rightarrow T$ gives rise to an action of $P$ on $X \times G$ and the quotient $Y:=(X \times G) / P$ is again a nonsingular projective variety over $F$. There is a canonical morphism $\pi: Y \rightarrow W:=P \backslash G$ such that $Y$ becomes a locally trivial fiber bundle over $W$ with fiber $X$. Corresponding to a character $\lambda \in X^{*}(P)$, there is a line bundle $L_{\lambda}$ on $W$ and the assignment $\lambda \mapsto L_{\lambda}$ gives an isomorphism $X^{*}(P) \rightarrow \operatorname{Pic}(W)$.

The toric variety $X$ can be described combinatorially by a fan $\Sigma$ in the dual space of the space of characters $X^{*}(T)_{\mathbf{R}}$. Let $P L(\Sigma)$ be the group of $\Sigma$-piecewise linear integral functions on the dual space of $X^{*}(T)_{\mathbf{R}}$. Any $\varphi \in P L(\Sigma)$ defines a line bundle $L_{\varphi}$ on $X$ which is equipped with a canonical $T$-linearization and we get an isomorphism $P L(\Sigma) \simeq$ $\operatorname{Pic}^{T}(X)$. There is a canonical exact sequence

$$
0 \rightarrow X^{*}(T) \rightarrow P L(\Sigma) \rightarrow \operatorname{Pic}(X) \rightarrow 0
$$

The $T$-linearization of $L_{\varphi}$ allows us to define a line bundle $L_{\varphi}^{Y}$ on $Y$ and this gives a homomorphism $\operatorname{PL}(\Sigma) \rightarrow \operatorname{Pic}(Y)$. One can show that there is an exact sequence

$$
0 \rightarrow X^{*}(T) \rightarrow P L(\Sigma) \oplus X^{*}(P) \rightarrow \operatorname{Pic}(Y) \rightarrow 0
$$

Denote by $Y^{o}:=(T \times G) / P$ the open subvariety of $Y$ obtained as the twist of $T$ with $W$.

1.6 By means of a maximal compact subgroup in the adelic group $G(\mathbf{A})$ we can introduce metrics on the line bundles $L_{\lambda}$. The corresponding height zeta functions are Eisenstein series

$$
\sum_{w \in W(F)} H_{\mathcal{L}_{\lambda}}(w)^{-s}=E_{P}^{G}\left(s \lambda-\rho_{P}, 1_{G}\right) .
$$

On the other hand, for any $\varphi \in P L(\Sigma)$ there is a function

$$
H_{\Sigma}(\cdot, \varphi): T(\mathbf{A}) \rightarrow \mathbf{R}_{>0}
$$

such that $H_{\Sigma}(x, \varphi)^{-1}$ is the height of $x \in T(F)$ with respect to a metrization $\mathcal{L}_{\varphi}$ of $L_{\varphi}$. This metrization induces a metrization $\mathcal{L}_{\varphi}^{Y}$ of the line bundle $L_{\varphi}^{Y}$ on $Y$. 
Let $(x, \gamma) \in T(F) \times G(F)$ and let $y$ be the image of $(x, \gamma)$ in $Y(F)$. Then there is a $p_{\gamma} \in P(\mathbf{A})$ such that

$$
H_{\mathcal{L}_{\varphi}^{Y}}(y)=H_{\Sigma}\left(x \eta\left(p_{\gamma}\right), \varphi\right)^{-1} .
$$

Hence we may write formally

$$
Z_{Y^{o}}\left(\mathcal{L}_{\varphi}^{Y} \otimes \pi^{*} \mathcal{L}_{\lambda}, s\right)=\sum_{\gamma \in P(F) \backslash G(F)} H_{\mathcal{L}_{\lambda}}(\gamma)^{-s} \sum_{x \in T(F)} H_{\Sigma}\left(x \eta\left(p_{\gamma}\right), s \varphi\right) .
$$

Now we apply Poisson's summation formula for the the torus and get

$$
\sum_{x \in T(F)} H_{\Sigma}\left(x \eta\left(p_{\gamma}\right), s \varphi\right)=\int_{(T(\mathbf{A}) / T(F))^{*}} \hat{H}_{\Sigma}(\chi, s \varphi) \chi\left(\eta\left(p_{\gamma}\right)\right)^{-1} d \chi
$$

where $\hat{H}_{\Sigma}(\cdot, s \varphi)$ denotes the Fourier transform of $H_{\Sigma}(\cdot, s \varphi)$ and $(T(\mathbf{A}) / T(F))^{*}$ is the group of unitary characters of $T(\mathbf{A})$ which are trivial on $T(F)$ equipped with the orthogonal measure $d \chi$. Actually, it is sufficient to consider only those characters that are trivial on the maximal compact subgroup $\mathbf{K}_{T}$ of $T(\mathbf{A})$, because the function $H_{\Sigma}(\cdot, \varphi)$ is invariant under $\mathbf{K}_{T}$. The expression (3) can now be put into (2), and after interchanging summation and integration the result is

$$
Z_{Y^{o}}\left(\mathcal{L}_{\varphi}^{Y} \otimes \pi^{*} \mathcal{L}_{\lambda}, s\right)=\int_{\left(T(\mathbf{A}) / T(F) \mathbf{K}_{T}\right)^{*}} \hat{H}_{\Sigma}(\chi, s \varphi) E_{P}^{G}\left(s \lambda-\rho_{P},(\chi \circ \eta)^{-1}\right) d \chi
$$

where $E_{P}^{G}\left(s \lambda-\rho_{P}, \xi\right)=E_{P}^{G}\left(s \lambda-\rho_{P}, \xi, 1_{G}\right)$ is the Eisenstein series twisted by a character $\xi$ of $P(\mathbf{A})$. This is the starting point for the investigation of the height zeta function. To get an expression that is more suited for our study, we decompose the group of characters $\left(T(\mathbf{A}) / T(F) \mathbf{K}_{T}\right)^{*}$ into a continuous and a discrete part, i.e.,

$$
\left(T(\mathbf{A}) / T(F) \mathbf{K}_{T}\right)^{*}=X^{*}(T)_{\mathbf{R}} \oplus \mathcal{U}_{T}
$$

where $X^{*}(T)_{\mathbf{R}}$ is the continuous part and $\mathcal{U}_{T}$ is the discrete part. The right-hand side of (4) is accordingly

$$
\int_{X^{*}(T)_{\mathbf{R}}}\left\{\sum_{\chi \in \mathcal{U}_{T}} \hat{H}_{\Sigma}(\chi, s \varphi+i x) E_{P}^{G}\left(s \lambda-\rho_{P}-i \check{\eta}(x),(\chi \circ \eta)^{-1}\right)\right\} d x .
$$

Recall that we would like to show that this function which is defined for $\operatorname{Re}(s) \gg 0$ (assuming that $(\varphi, \lambda)$ is contained in a convex open cone) can be continued meromorphically 
beyond the abscissa of convergence. To achieve this we need more information on the function under the integral sign in (5). First we have to determine the singularities of

$$
(\varphi, \lambda) \mapsto \hat{H}_{\Sigma}(\chi, \varphi) E_{P}^{G}\left(\lambda-\rho_{P},(\chi \circ \eta)^{-1}\right)
$$

near the cone of absolute convergence. This is possible because $\hat{H}_{\Sigma}$ can be calculated rather explicitly and it is not so difficult to determine the singular hyperplanes of the Eisenstein series with characters. The next step consists in an iterated application of Cauchy's residue formula to the integral over the real vector space $X^{*}(T)_{\mathbf{R}}$. This can be done only if one knows that

$$
\sum_{\chi \in \mathcal{U}_{T}} \hat{H}_{\Sigma}(\chi, s \varphi+i x) E_{P}^{G}\left(s \lambda-\rho_{P}-i \check{\eta}(x),(\chi \circ \eta)^{-1}\right)
$$

satisfies some growth conditions when $x \in X^{*}(T)_{\mathbf{R}}$ tends to infinity. This is true for the function $x \mapsto \hat{H}_{\Sigma}(\chi, s \varphi+i x)$ thanks to the explicit expression mentioned above. The absolute value of the Eisenstein series $E_{P}^{G}\left(s \lambda-\rho_{P}-i \check{\eta}(x),(\chi \circ \eta)^{-1}\right)$ will in general increase for $x \rightarrow \infty$ if $\operatorname{Re}(s) \lambda-\rho_{P}$ is not contained in the cone of absolute convergence. However, if $\operatorname{Re}(s) \lambda-\rho_{P}$ is sufficiently close to the boundary of that cone, this increasing behavior is absorbed by the decreasing behavior of $\hat{H}_{\Sigma}(\chi, s \varphi+i x)$.

Therefore, we may apply Cauchy's residue theorem and show that (4) can be continued meromorphically to a larger halfspace and that there are no poles (in $s$ ) with a non-zero imaginary part.

The Tauberian Theorem can now be used to prove asymptotic formulas for the counting function $N_{Y^{o}}\left(\mathcal{L}_{\varphi}^{Y} \otimes \pi^{*} \mathcal{L}_{\lambda}, H\right)$ provided that one knows the order of the pole of the height zeta function. This problem can be reduced to the question of whether or not the "leading term" of the Laurent series of (6) vanishes. That this is indeed so will be shown in section 6.

1.7 We have restricted ourself to the case of split tori and split groups because this simplifies some technical details. The general case can be treated similarly.

We consider these results as an important step towards an understanding of the arithmetic of spherical varieties. For example, choosing $P=B$ a Borel subgroup, $T=U \backslash B$ where $U$ is the unipotent radical of $B$ and $\eta: B \rightarrow T$ the natural projection, we obtain an equivariant compactification of $U \backslash G$, a horospherical variety. Twisted products over $P \backslash G$ have also been studied in [44], where the fiber is a flag variety (of a different type). In this situation the asymptotic behavior turns out to be the predicted one. 
We close this introduction with a brief description of the remaining sections. Section 2 recalls the relevant facts we need concerning generalized flag varieties, i.e., a description of line bundles on $W=P \backslash G$, the cone of effective divisors in $\operatorname{Pic}(W)_{\mathbf{R}}$, metrization of line bundles, height zeta functions. The exposition is based entirely on the paper [14].

The next section contains the corresponding facts for toric varieties. It is a summary of a part of [3]. We give the explicit calculation of the Fourier transform $\hat{H}_{\Sigma}(\cdot, \varphi)$ and show that Poisson's summation formula can be used to give an expression of the height zeta function $Z_{T}\left(\mathcal{L}_{\varphi}, s\right)$.

In section 4 we introduce twisted products, discuss line bundles on them, the Picard group (cf., (1)), metrizations of line bundles, etc. It ends with the formula (4) for the height zeta function $Z_{Y^{o}}\left(\mathcal{L}_{\varphi}^{Y} \otimes \pi^{*} \mathcal{L}_{\lambda}, s\right)$ in the domain of absolute convergence.

The first part of section 5 explains the method for the proof that the height zeta function can be continued meromorphically to a halfspace beyond the abscissa of absolute convergence. Moreover, we state a theorem which gives a description of the coefficient of the Laurent series at the pole in question. This coefficient will be the leading one, provided that it does not vanish. One can relate the coefficient to arithmetic and geometric invariants of the pair $(U, \mathcal{L})$ but we decided not to pursue this, since there are detailed expositions of all the necessary arguments in $[31,3,7]$.

These two theorems (meromorphic continuation of certain integrals and the description of the coefficient) will be proved in a more general context in section 7 . The second part of section 5 contains the proof that the hypothesis of these theorems are fulfilled in our case. It ends with the main theorem on the asymptotic behavior of the counting function $N_{Y^{o}}(\mathcal{L}, H)$, assuming that the coefficient of the Laurent series mentioned above does not vanish. Section 6 is devoted to the proof of this fact. In section 8 we prove some statements on Eisenstein series (well-known to the experts) which are used in section 5 . And finally, in section 9 we explain in detail some special cases of our main theorem.

Acknowledgements. We are very grateful to V. Batyrev and J. Franke for helpful discussions. The first author was supported by the DFG-Graduiertenkolleg of the Mathematics Institute of the University of Bonn. He wants to thank the organizers of conferences in Oberwolfach (July 1997) and Cambridge (April 1998) for giving him the opportunity to present these and related results. Part of this work was done while the second author was visiting the MPI in Bonn, ETH Zürich and ENS Paris. He would like to thank these institutions for their hospitality.

Some notations. In this paper $F$ always denotes a fixed algebraic number field. The set of places of $F$ will be denoted by $\operatorname{Val}(F)$ and the subset of archimedean places by $\operatorname{Val}_{\infty}(F)$. We shall write $v \mid \infty$ if $v \in \operatorname{Val}_{\infty}(F)$ and $v \nmid \infty$ if $v \notin \operatorname{Val}_{\infty}(F)$. For any place 
$v$ of $F$ we denote by $F_{v}$ the completion of $F$ at $v$ and by $\mathcal{O}_{v}$ the ring of $v$-adic integers (for $v \nmid \infty$ ). The local absolute value $|\cdot|_{v}$ on $F_{v}$ is the multiplier of the Haar measure, i.e., $d\left(a x_{v}\right)=|a|_{v} d x_{v}$ for some Haar measure $d x_{v}$ on $F_{v}$. Let $q_{v}$ be the cardinality of the residue field of $F_{v}$ for non-archimedean valuations and put $q_{v}=e$ for archimedean valuations. We denote by $\mathbf{A}$ the adele ring of $F$. For any algebraic group $G$ over $F$ we denote by $X^{*}(G)$ the group of (algebraic) characters which are defined over $F$.

\section{Generalized flag varieties}

2.1 Let $G$ be a semi-simple simply connected linear algebraic group which is defined and split over $F$. We fix a Borel subgroup $P_{0}$ over $F$ and a Levi decomposition $P_{0}=S_{0} U_{0}$ with a maximal $F$-rational torus $S_{0}$ of $G$. Denote by $P$ a standard (i.e., containing $\left.P_{0}\right)$ parabolic subgroup and by $W=P \backslash G$ the corresponding flag variety. The quotient morphism $G \rightarrow W$ will be denoted by $\pi_{W}$. A character $\lambda \in X^{*}(P)$ gives rise to an action of $P$ on $G \times \mathbf{A}^{1}$ by $p \cdot(g, a)=\left(p g, \lambda(p)^{-1} a\right)$ and the quotient

$$
L_{\lambda}:=P \backslash\left(G \times \mathbf{A}^{1}\right)
$$

is a line bundle $L_{\lambda}$ over $W=P \backslash G$. The class of a point $(g, a) \in\left(G \times \mathbf{A}^{1}\right)$ in $L_{\lambda}$ will be denoted by $[g, a]$.

The assignment $\lambda \mapsto L_{\lambda}$ gives an isomorphism (because $G$ is assumed to be simply connected)

$$
X^{*}(P) \rightarrow \operatorname{Pic}(W)
$$

(cf. [37], Prop. 6.10). The anti-canonical line bundle $\omega_{W}^{\vee}$ corresponds to $2 \rho_{P}$ (the sum of roots of $S_{0}$ occurring in the Lie algebra of the unipotent radical of $P$.)

2.2 These line bundles will be metrized as follows. Choose a maximal compact subgroup $\mathbf{K}_{G}=\prod_{v} \mathbf{K}_{G, v} \subset G(\mathbf{A})\left(\mathbf{K}_{G, v} \subset G\left(F_{v}\right)\right)$, such that the Iwasawa decomposition

$$
G(\mathbf{A})=P_{0}(\mathbf{A}) \mathbf{K}_{G}
$$


holds. Let $v \in \operatorname{Val}(F)$ and $w \in W\left(F_{v}\right)$. Choose $k \in \mathbf{K}_{G, v}$ which is mapped to $w$ by $\pi_{W}$. For any $F_{v}$-rational point $[g, a] \in L_{\lambda}\left(F_{v}\right)$ over $w$ with $(g, a) \in G\left(F_{v}\right) \times \mathbb{A}^{1}\left(F_{v}\right)$, we define

$$
\|[g, a]\|_{w}:=|\lambda(p) \cdot a|_{v},
$$

where $g=p k, p \in P\left(F_{v}\right)$. This gives a $v$-adic norm $\|\cdot\|_{w}: w^{*} L_{\lambda} \rightarrow \mathbf{R}$ and we see that the family $\|\cdot\|_{v}:=\left(\|\cdot\|_{w}\right)_{w \in W\left(F_{v}\right)}$ is a $v$-adic metric on $L_{\lambda}$. The family $\left(\|\cdot\|_{v}\right)_{v \in \operatorname{Val}(F)}$ will then be an adelic metric on $L_{\lambda}$ (cf. [31] for $\lambda=2 \rho_{P}$ and [45] for the definitions of " $v$-adic metric" and "adelic metric"). The metrized line bundle $\left(L_{\lambda},\left(\|\cdot\|_{v}\right)_{v}\right)$ will be denoted by $\mathcal{L}_{\lambda}$.

2.3 Define a map

$$
H_{P}=H_{P, \mathbf{K}_{G}}: G(\mathbf{A}) \rightarrow \operatorname{Hom}_{\mathbf{C}}\left(X^{*}(P)_{\mathbf{C}}, \mathbf{C}\right)
$$

by $\left\langle\lambda, H_{P}(g)\right\rangle=\log \left(\prod_{v}\left|\lambda\left(p_{v}\right)\right|_{v}\right)$ for $g=p k$ with $p=\left(p_{v}\right)_{v} \in P(\mathbf{A}), k \in \mathbf{K}_{G}$ and $\lambda \in X^{*}(P)$. For $w=\pi_{W}(\gamma) \in W(F)$ and $\gamma \in G(F)$ a simple computation ([14], p. 428) shows that

$$
H_{\mathcal{L}_{\lambda}}(w)=e^{-\left\langle\lambda, H_{P}(\gamma)\right\rangle} .
$$

The height zeta function

$$
Z_{W}\left(\mathcal{L}_{\lambda}, s\right)=\sum_{w \in W(F)} H_{\mathcal{L}_{\lambda}}(w)^{-s}
$$

is therefore an Eisenstein series

$$
E_{P}^{G}\left(s \lambda-\rho_{P}, 1_{G}\right)=\sum_{\gamma \in P(F) \backslash G(F)} e^{\left\langle s \lambda, H_{P}(\gamma)\right\rangle} .
$$

To describe the domain of absolute convergence of this series we let $\Delta_{0}$ be the basis of positive roots of the root system $\Phi\left(S_{0}, G\right)$ which is determined by $P_{0}$. For any $\alpha \in \Delta_{0}$ denote by $\check{\alpha}$ the corresponding coroot. For $\lambda \in X^{*}(P)=X^{*}\left(S_{0}\right)$ we define $\langle\lambda, \alpha\rangle$ by $(\lambda \circ \check{\alpha})(t)=t^{\langle\lambda, \alpha\rangle}$ and extend this linearly in $\lambda$ to $X^{*}\left(P_{0}\right)_{\mathbf{C}}$. Restriction of characters defines an inclusion $X^{*}(P) \rightarrow X^{*}\left(P_{0}\right)$. Let

$$
\Delta_{0}^{P}=\left\{\alpha \in \Delta_{0} \mid\langle\cdot, \alpha\rangle \text { vanishes on } X^{*}(P)\right\}, \quad \Delta_{P}=\Delta_{0}-\Delta_{0}^{P} .
$$

Put

$$
X^{*}(P)^{+}=\left\{\lambda \in X^{*}(P)_{\mathbf{R}} \mid\langle\lambda, \alpha\rangle>0 \text { for all } \alpha \in \Delta_{P}\right\} .
$$


By [16], Théorème 3, the Eisenstein series

$$
E_{P}^{G}(\lambda, g)=\sum_{\gamma \in P(F) \backslash G(F)} e^{\left\langle\lambda+\rho_{P}, H_{P}(\gamma g)\right\rangle}
$$

converges absolutely for $\operatorname{Re}(\lambda)-\rho_{P}$ in $X^{*}(P)^{+}$and it can be meromorphically continued to $X^{*}(P)_{\mathbf{C}}$ (cf. [27], IV, 1.8$)$. The closure of the image of $X^{*}(P)^{+}$in $\operatorname{Pic}(W)_{\mathbf{R}}$ is the cone $\Lambda_{\text {eff }}(W)$ generated by the effective divisors on $W([19]$, II, 2.6).

\section{Toric varieties}

3.1 Let $T$ be a split algebraic torus of dimension $d$ over $F$. We put $M=X^{*}(T)$ and $N=\operatorname{Hom}(M, \mathbf{Z})$. Let $\Sigma$ be a complete regular fan in $N_{\mathbf{R}}$ such that the corresponding smooth toric variety $X=X_{\Sigma}$ is projective (cf. [15, 30]). The variety $X$ is covered by affine open sets

$$
U_{\sigma}=\operatorname{Spec}(F[M \cap \check{\sigma}])
$$

where $\sigma$ runs through $\Sigma$ and $\check{\sigma}$ is the dual cone

$$
\check{\sigma}=\left\{m \in M_{\mathbf{R}} \mid n(m) \geq 0 \forall n \in \sigma\right\} .
$$

Denote by $P L(\Sigma)$ the group of $\Sigma$-piecewise linear integral functions on $N_{\mathbf{R}}$. By definition, a function $\varphi: N_{\mathbf{R}} \rightarrow \mathbf{R}$ belongs to $P L(\Sigma)$ if and only if $\varphi(N) \in \mathbf{Z}$ and the restriction of $\varphi$ to every $\sigma \in \Sigma$ is the restriction to $\sigma$ of a linear function on $N_{\mathbf{R}}$. For $\varphi \in P L(\Sigma)$ and every $d$-dimensional cone $\sigma \in \Sigma$ there exists a unique $m_{\varphi, \sigma} \in M$ such that for all $n \in \sigma$ we have

$$
\varphi(n)=n\left(m_{\varphi, \sigma}\right) .
$$

Fixing for any $\sigma \in \Sigma$ a $d$-dimensional cone $\sigma^{\prime}$ containing $\sigma$, we put

$$
m_{\varphi, \sigma}=m_{\varphi, \sigma^{\prime}}
$$

To any $\varphi \in P L(\Sigma)$ we associate a line bundle $L_{\varphi}$ on $X$ as follows:

$$
L_{\varphi}=\left(\coprod_{\sigma \in \Sigma} U_{\sigma} \times \mathbb{A}^{1}\right) / \sim
$$

where

$$
U_{\sigma} \times \mathbb{A}^{1} \ni(x, a) \sim\left(x^{\prime}, a^{\prime}\right) \in U_{\sigma^{\prime}} \times \mathbb{A}^{1}
$$




$$
\Leftrightarrow\left(x=x^{\prime} \in U_{\sigma} \cap U_{\sigma^{\prime}} \text {, and } a^{\prime}=\frac{m_{\varphi, \sigma^{\prime}}}{m_{\varphi, \sigma}}(x) \cdot a\right) \text {. }
$$

The class of a point $(x, a) \in U_{\sigma} \times \mathbb{A}^{1}$ in $L_{\varphi}$ will be denoted by $[x, a]$. On $L_{\varphi}$ there is a canonical $T$-action $L_{\varphi} \times T \rightarrow L_{\varphi}$, compatible with the action of $T$ on $X$ given by

$$
[x, a] \cdot t=\left[x \cdot t, m_{\varphi, \sigma}(t) \cdot a\right], \text { if }(x, a) \in U_{\sigma} \times \mathbb{A}^{1} .
$$

If we consider $L_{\varphi}$ with this fixed "T-linearization", then $P L(\Sigma)$ becomes isomorphic to the group $\operatorname{Pic}^{T}(X)$ of isomorphism classes of $T$-linearized line bundles on $X$ (cf. [28], Ch. $1, \S 3)$. By [30], Corollary 2.5, the assignment $\varphi \rightarrow L_{\varphi}$ gives rise to an exact sequence

$$
0 \rightarrow M \rightarrow P L(\Sigma) \rightarrow \operatorname{Pic}(X) \rightarrow 0
$$

Let $\Sigma_{1} \subset N$ be the set of primitive integral generators of the one-dimensional cones in $\Sigma$ and put

$$
P L(\Sigma)^{+}:=\left\{\varphi \in P L(\Sigma)_{\mathbf{R}} \mid \varphi(e)>0 \text { for all } e \in \Sigma_{1}\right\} .
$$

It is well-known (cf. [35], [3] Prop. 1.2.11), that the cone of effective divisors $\Lambda_{\text {eff }}(X) \subset$ $\operatorname{Pic}(X)_{\mathbf{R}}$ is the closure of the image of $P L(\Sigma)^{+}$under the projection $P L(\Sigma)_{\mathbf{R}} \rightarrow \operatorname{Pic}(X)_{\mathbf{R}}$. Further, the anti-canonical line bundle on $X$ is isomorphic to $L_{\varphi_{\Sigma}}$, where $\varphi_{\Sigma}(e)=1$ for all $e \in \Sigma_{1}$ (cf. [3], Prop. 1.2.12).

3.2 We shall introduce an adelic metric on the line bundle $L_{\varphi}$ as follows. For $\sigma \in \Sigma$ and $v \in \operatorname{Val}(F)$ define

$$
\mathbf{K}_{\sigma, v}:=\left\{\left.x \in U_{\sigma}\left(F_{v}\right)|| m(x)\right|_{v} \leq 1 \forall m \in \check{\sigma} \cap M\right\}
$$

These subsets cover $X\left(F_{v}\right)$. Let $x \in \mathbf{K}_{\sigma, v}$ and $a \in F_{v}$. Then $[x, a] \in L_{\varphi}\left(F_{v}\right)$ is an $F_{v}$-rational point of $L_{\varphi}$ over $x$. We put

$$
\|[x, a]\|_{x}:=|a|_{v} .
$$

This is well-defined because for $x \in \mathbf{K}_{\sigma, v} \cap \mathbf{K}_{\sigma^{\prime}, v}$, we have $\left|\frac{m_{\varphi, \sigma^{\prime}}}{m_{\varphi, \sigma}}(x)\right|_{v}=1$. The family $\|\cdot\|_{v}=\left(\|\cdot\|_{x}\right)_{x \in X\left(F_{v}\right)}$ is then a $v$-adic metric on $L_{\varphi}$ and $\mathcal{L}_{\varphi}=\left(L_{\varphi},\left(\|\cdot\|_{v}\right)_{v}\right)$ is a metrization of $L_{\varphi}$. Let $\mathbf{K}_{T, v} \subset T\left(F_{v}\right)$ be the maximal compact subgroup. Assigning to $x \in T\left(F_{v}\right)$ the map

$$
M \rightarrow \mathbf{Z}(\text { resp. } \mathbf{R} \text { if } v \mid \infty)
$$




$$
m \mapsto-\frac{\log \left(|m(x)|_{v}\right)}{\log \left(q_{v}\right)},
$$

(where $q_{v}$ is the order of the residue field of $F_{v}$ for non-archimedean valuations and $\log \left(q_{v}\right)=1$ for archimedean valuations) we get an isomorphism $T\left(F_{v}\right) / \mathbf{K}_{T, v} \rightarrow N$ (resp. $N_{\mathbf{R}}$ if $v \mid \infty$ ). We will denote by $\bar{x}$ the image of $x \in T\left(F_{v}\right)$ in $N$ (resp. $N_{\mathbf{R}}$ ). For $\varphi \in P L(\Sigma)_{\mathbf{C}}$ define

$$
\begin{aligned}
& H_{\Sigma, v}(\cdot, \varphi): T\left(F_{v}\right) \rightarrow \mathbf{C}, \\
& H_{\Sigma, v}(x, \varphi):=e^{-\varphi(\bar{x}) \log \left(q_{v}\right)} .
\end{aligned}
$$

The corresponding global function $H_{\Sigma}(\cdot, \varphi): T(\mathbf{A}) \rightarrow \mathbf{C}$,

$$
H_{\Sigma}(x, \varphi):=\prod_{v} H_{\Sigma, v}\left(x_{v}, \varphi\right),
$$

is well defined since, for almost all $v$, the local component $x_{v}$ belongs to $\mathbf{K}_{T, v}$. The functions $H_{\Sigma, v}(\cdot, \varphi), \varphi \in P L(\Sigma)$, are related to the $v$-adic metric on $L_{\varphi}$ by the identity

$$
H_{\Sigma, v}(x, \varphi)=\left\|\left[x, m_{\varphi, \sigma}(x)\right]\right\|_{x}, \quad\left(x \in T\left(F_{v}\right)\right),
$$

where we consider $\left(x, m_{\varphi, \sigma}(x)\right)$ as an element of $U_{\sigma}\left(F_{v}\right) \times F_{v}$. In particular, for every $x \in T(F)$ we have

$$
H_{\mathcal{L}_{\varphi}}(x)=H_{\Sigma}(x, \varphi)^{-1} .
$$

3.3 Let $\mathbf{K}_{T}=\prod_{v} \mathbf{K}_{T, v} \subset T(\mathbf{A})$, and denote by

$$
\mathcal{A}_{T}=\left(T(\mathbf{A}) / T(F) \mathbf{K}_{T}\right)^{*}
$$

the group of unitary characters of $T(\mathbf{A})$ which are trivial on the closed subgroup $T(F) \mathbf{K}_{T}$. For $m \in M$ we obtain characters $\chi^{m}$ defined by

$$
\chi^{m}(x):=e^{i \log \left(|m(x)|_{\mathbf{A}}\right)} .
$$

This gives an embedding $M_{\mathbf{R}} \rightarrow \mathcal{A}_{T}$. For any archimedean place $v$ and $\chi \in \mathcal{A}_{T}$, there is an $m_{v}=m_{v}(\chi) \in M_{\mathbf{R}}$ such that $\chi_{v}\left(x_{v}\right)=e^{-i \bar{x}_{v}\left(m_{v}\right)}$ for all $x_{v} \in T\left(F_{v}\right)$. We get a homomorphism

$$
\mathcal{A}_{T} \rightarrow M_{\mathbf{R}, \infty}=\oplus_{v \mid \infty} M_{\mathbf{R}}
$$




$$
\chi \mapsto m_{\infty}(\chi)=\left(m_{v}(\chi)\right)_{v \mid \infty}
$$

Define $T(\mathbf{A})^{1}$ to be the kernel of all maps $T(\mathbf{A}) \rightarrow \mathbf{R}_{>0}, x \mapsto|m(x)|_{\mathbf{A}}$, for $m \in M$, and put

$$
\mathcal{U}_{T}=\left(T(\mathbf{A})^{1} / T(F) \mathbf{K}_{T}\right)^{*} .
$$

The choice of a projection $\mathbf{G}_{m}(\mathbf{A}) \rightarrow \mathbf{G}_{m}(\mathbf{A})^{1}$ induces by means of an isomorphism $T \stackrel{\sim}{\longrightarrow} \mathbf{G}_{m, F}^{d}$ a splitting of the exact sequence

$$
1 \rightarrow T(\mathbf{A})^{1} \rightarrow T(\mathbf{A}) \rightarrow T(\mathbf{A}) / T(\mathbf{A})^{1} \rightarrow 1
$$

This gives decompositions

$$
\mathcal{A}_{T}=M_{\mathbf{R}} \oplus \mathcal{U}_{T}
$$

and

$$
M_{\mathbf{R}, \infty}=M_{\mathbf{R}} \oplus M_{\mathbf{R}, \infty}^{1},
$$

where $M_{\mathbf{R}, \infty}^{1}$ is the minimal $\mathbf{R}$-subspace of $M_{\mathbf{R}, \infty}$ containing the image of $\mathcal{U}_{T}$ under the map (8). From now on we fix such a (non-canonical) splitting. By Dirichlet's unit theorem, the image of $\mathcal{U}_{T} \rightarrow M_{\mathbf{R}, \infty}^{1}$ is a lattice of maximal rank. Its kernel is isomorphic to the character group of $\mathrm{Cl}_{F}^{d}$, where $\mathrm{Cl}_{F}$ is the ideal class group of $F$.

For finite $v$ we let $d x_{v}$ be the Haar measure on $T\left(F_{v}\right)$ giving $\mathbf{K}_{T, v}$ the volume one. For archimedean $v$ we take on $T\left(F_{v}\right) / \mathbf{K}_{T, v}$ the pull-back of the Lebesgue measure on $N_{\mathbf{R}}$ (normalized by the lattice $N$ ) and on $\mathbf{K}_{T, v}$ the Haar measure with total mass one. The product measure gives an invariant measure $d x_{v}$ on $T\left(F_{v}\right)$. On $T(\mathbf{A})$ we get a Haar measure $d x=\prod_{v} d x_{v}$.

3.4 We will denote by $S^{1}$ the unit circle. For a character $\chi: T\left(F_{v}\right) \rightarrow S^{1}$ we define the Fourier transform of $H_{\Sigma, v}(\cdot, \varphi)$ by

$$
\hat{H}_{\Sigma, v}(\chi, \varphi)=\int_{T\left(F_{v}\right)} H_{\Sigma, v}\left(x_{v}, \varphi\right) \chi\left(x_{v}\right) d x_{v} .
$$

If $\chi$ is not trivial on $\mathbf{K}_{T, v}$ then $\hat{H}_{\Sigma, v}(\chi, \varphi)=0$ (assuming the convergence of the integral). We will show that these integrals do exists if $\operatorname{Re}(\varphi)$ is in $P L(\Sigma)^{+}$. 
Let $v$ be an archimedean place of $F$. Any $d$-dimensional cone $\sigma \in \Sigma$ is simplicial (since $\Sigma$ is regular) and it is generated by the set $\sigma \cap \Sigma_{1}$. Let $\chi$ be unramified, i.e., $\chi(x)=e^{-i \bar{x}(m)}$ with some $m \in M_{\mathbf{R}}$. Then we get

$$
\hat{H}_{\Sigma, v}(\chi, \varphi)=\sum_{\operatorname{dim} \sigma=d} \int_{\sigma} e^{-(\varphi(n)+i n(m))} d n=\sum_{\operatorname{dim} \sigma=d} \prod_{e \in \sigma \cap \Sigma_{1}} \frac{1}{\varphi(e)+i e(m)} .
$$

To give the result for finite places we define rational functions $R_{\sigma}$ in variables $u_{e}, e \in \Sigma_{1}$, for any $\sigma \in \Sigma$ by

$$
R_{\sigma}\left(\left(u_{e}\right)_{e}\right)=\prod_{e \in \sigma \cap \Sigma_{1}} \frac{u_{e}}{1-u_{e}}
$$

and put

$$
\begin{gathered}
R_{\Sigma}\left(\left(u_{e}\right)_{e}\right)=\sum_{\sigma \in \Sigma} R_{\sigma}\left(\left(u_{e}\right)_{e}\right), \\
Q_{\Sigma}\left(\left(u_{e}\right)_{e}\right)=R_{\Sigma}\left(\left(u_{e}\right)_{e}\right) \prod_{e \in \Sigma_{1}}\left(1-u_{e}\right) .
\end{gathered}
$$

Although elementary, it is a very important observation that the polynomial $Q_{\Sigma}-1$ is a sum of monomials of degree not less than two (cf. [3], Prop. 2.2.3).

Let $\chi$ be an unramified unitary character of $T\left(F_{v}\right)$ and let $\operatorname{Re}(\varphi)$ be in $P L(\Sigma)^{+}$. Then we can calculate

$$
\begin{aligned}
\hat{H}_{\Sigma, v}(\chi, \varphi) & =\int_{T\left(F_{v}\right)} H_{\Sigma, v}\left(x_{v}, \varphi\right) \chi\left(x_{v}\right) d x_{v}=\sum_{n \in N} e^{-\varphi(n) \log \left(q_{v}\right)} \chi(n) \\
& =\sum_{\sigma \in \Sigma} \sum_{n \in \sigma^{\circ} \cap N} q_{v}^{-\varphi(n)} \chi(n)=\sum_{\sigma \in \Sigma} R_{\sigma}\left(\left(\chi(e) q_{v}^{-\varphi(e)}\right)_{e}\right) \\
& =Q_{\Sigma}\left(\left(\chi(e) q_{v}^{-\varphi(e)}\right)_{e}\right) \prod_{e \in \Sigma_{1}}\left(1-\chi(e) q_{v}^{-\varphi(e)}\right)^{-1} .
\end{aligned}
$$

(Here we denoted by $\sigma^{o}$ the relative interior of the cone $\sigma$.)

Any $e \in \Sigma_{1}$ induces a homomorphism $F[M] \rightarrow F[\mathbf{Z}]$ and hence a morphism of tori $\mathbf{G}_{m} \rightarrow T$. For any character $\chi \in \mathcal{A}_{T}$ we denote by $\chi_{e}$ the Hecke character

$$
\mathbf{G}_{m}(\mathbf{A}) \longrightarrow T(\mathbf{A}) \stackrel{\chi}{\longrightarrow} S^{1}
$$

thus obtained. The finite part of the Hecke $L$-function with character $\chi_{e}$ is by definition

$$
L_{f}\left(\chi_{e}, s\right)=\prod_{v \nmid \infty}\left(1-\chi_{e}\left(\pi_{v}\right) q_{v}^{s}\right)^{-1}
$$


and this product converges for $\operatorname{Re}(s)>1$ (here $\pi_{v}$ denotes a local uniformizing element). By (10) and (11) we know that the global Fourier transform

$$
\hat{H}_{\Sigma}(\chi, \varphi)=\int_{T(\mathbf{A})} H_{\Sigma}(x, \varphi) \chi(x) d x
$$

exists (i.e., the integral on the right converges absolutely) if $\operatorname{Re}(\varphi)$ is contained in $\varphi_{\Sigma}+$ $P L(\Sigma)^{+}$, because

$$
\prod_{v \nmid \infty} Q_{\Sigma}\left(\left(\chi_{v}(e) q_{v}^{-\varphi(e)}\right)_{e}\right)
$$

is an absolutely convergent Euler product for $\operatorname{Re}(\varphi(e))>1 / 2$ (for all $e \in \Sigma_{1}$ ) and hence is bounded for $\operatorname{Re}(\varphi)$ in any compact subset in $\frac{1}{2} \varphi_{\Sigma}+P L(\Sigma)^{+}$(by some constant depending only on this subset).

Proposition 3.4 The series

$$
\sum_{x \in T(F)} H_{\Sigma}(x t, \varphi)
$$

converges absolutely and uniformly for $(\operatorname{Re}(\varphi), t)$ contained in any compact subset of $\left(\varphi_{\Sigma}+\right.$ $\left.P L(\Sigma)^{+}\right) \times T(\mathbf{A})$.

Proof. Let $\mathbf{K}$ be a compact subset of $\varphi_{\Sigma}+P L(\Sigma)^{+}$and let $C_{v} \subset T\left(F_{v}\right)$ (for every $v \in \operatorname{Val}(F)$ ) be a compact subset, equal to $\mathbf{K}_{T, v}$ for almost all $v$. Since any $\varphi \in P L(\Sigma)_{\mathbf{C}}$ is a continuous piecewise linear function (with respect to a finite subdivision of $N_{\mathbf{R}}$ into simplicial cones) there exists a constant $c_{v} \geq 1$ (depending on $\mathbf{K}$ and $C_{v}$ ) such that for all $\varphi$ with $\operatorname{Re}(\varphi) \in \mathbf{K}, x_{v} \in T\left(F_{v}\right)$ and $t_{v} \in C_{v}$ we have

$$
\frac{1}{c_{v}} \leq\left|\frac{H_{\Sigma, v}\left(x_{v} t_{v}, \varphi\right)}{H_{\Sigma, v}\left(x_{v}, \varphi\right)}\right|=\frac{H_{\Sigma, v}\left(x_{v} t_{v}, \operatorname{Re}(\varphi)\right)}{H_{\Sigma, v}\left(x_{v}, \operatorname{Re}(\varphi)\right)} \leq c_{v} .
$$

If $C_{v}=\mathbf{K}_{T, v}$ we may assume $c_{v}=1$. Put $c=\prod_{v} c_{v}$. For all $\varphi$ with $\operatorname{Re}(\varphi) \in \mathbf{K}$ and $t \in C:=\prod_{v} C_{v}$ we can estimate

$$
\left|\sum_{x \in T(F)} H_{\Sigma}(x t, \varphi)\right| \leq c \sum_{x \in T(F)} H_{\Sigma}(x, \operatorname{Re}(\varphi)) .
$$

Let $S$ be a finite set of places containing $\operatorname{Val}_{\infty}(F)$ and let $U_{v} \subset T\left(F_{v}\right)$ be a relatively compact open subset of $T\left(F_{v}\right)$ for each $v \in S$, such that for all $x_{1} \neq x_{2} \in T(F)$

$$
x_{1} U \cap x_{2} U=\emptyset,
$$


where $U=\prod_{v \in S} U_{v} \prod_{v \notin S} \mathbf{K}_{T, v}$. By the preceding argument, there exists a $c^{\prime}>0$ such that for all $\varphi \in \mathbf{K}, x \in T(F)$ and $u \in U$

$$
H_{\Sigma}(x, \varphi) \leq c^{\prime} H_{\Sigma}(x u, \varphi) .
$$

Therefore,

$$
\begin{gathered}
\sum_{x \in T(F)} H_{\Sigma}(x, \varphi) \leq \frac{c^{\prime}}{\operatorname{vol}(U)} \sum_{x \in T(F)} \int_{U} H_{\Sigma}(x u, \varphi) d u \\
\leq \frac{c^{\prime}}{\operatorname{vol}(U)} \int_{T(\mathbf{A})} H_{\Sigma}(x, \varphi) d x<\infty
\end{gathered}
$$

by the discussion above. From the explicit expression for the integral (cf. (12)) we derive the uniform convergence in $\varphi$ on $\mathbf{K}$.

3.5 The aim is to apply Poisson's summation formula to the height zeta function. It remains to show that $\hat{H}(\cdot, \varphi)$ is absolutely integrable over $\mathcal{A}_{T}$. For $\chi \in \mathcal{A}_{T}$ and $\operatorname{Re}(\varphi)$ contained in $\frac{1}{2} \varphi_{\Sigma}+P L(\Sigma)^{+}$, we put

$$
\zeta_{\Sigma}(\chi, \varphi):=\prod_{v \mid \infty} \hat{H}_{\Sigma, v}\left(\chi_{v}, \varphi\right) \prod_{v \nmid \infty} Q_{\Sigma}\left(\left(\chi_{v}(e) q_{v}^{-\varphi(e)}\right)_{e}\right) .
$$

Lemma 3.5 Let $\mathbf{K}$ be a compact subset of $P L(\Sigma)_{\mathbf{C}}$ such that for all $\varphi \in \mathbf{K}$ and $e \in \Sigma_{1}$

$$
\operatorname{Re}(\varphi(e))>\frac{1}{2} .
$$

Then there is a constant $c=c(\mathbf{K})$ such that for all $\varphi \in \mathbf{K}, \chi \in \mathcal{A}_{T}$ and $m \in M_{\mathbf{R}}$ we have

$$
\left|\zeta_{\Sigma}(\chi, \varphi+i m)\right| \leq c \prod_{v \mid \infty}\left\{\sum_{\operatorname{dim} \sigma=d} \prod_{e \in \sigma \cap \Sigma_{1}} \frac{1}{\left(1+\left|e\left(m+m_{v}(\chi)\right)\right|\right)^{1+1 / d}}\right\} .
$$

Proof. For $\mathbf{K}$ as above there exists a $c^{\prime}>0$ such that for all $\chi \in \mathcal{A}_{T}$ and $m \in M_{\mathbf{R}}$ one has

$$
\left|\prod_{v \nmid \infty} Q_{\Sigma}\left(\left(\chi_{v}(e) q_{v}^{-(\varphi(e)+i e(m))}\right)_{e}\right)\right| \leq c^{\prime}
$$


for all $\varphi \in \mathbf{K}$ (see the argument before Proposition 3.4. By [3], Prop. 2.3.2, for all $v \mid \infty$ there is a constant $c_{v}$ such that for all $\varphi \in \mathbf{K}, \chi \in \mathcal{A}_{T}$ and $m \in M_{\mathbf{R}}$

$$
\left|\hat{H}_{\Sigma, v}\left(\chi_{v}, \varphi+i m\right)\right| \leq c_{v} \sum_{\operatorname{dim} \sigma=d} \prod_{e \in \sigma \cap \Sigma_{1}} \frac{1}{\left(1+\left|e\left(m+m_{v}(\chi)\right)\right|\right)^{1+1 / d}} .
$$

Putting $c=c^{\prime} \prod_{v \mid \infty} c_{v}$ we get the result.

For $\operatorname{Re}(\varphi)$ contained in $\varphi_{\Sigma}+P L(\Sigma)^{+}$we can write

$$
\hat{H}_{\Sigma}(\chi, \varphi)=\zeta_{\Sigma}(\chi, \varphi) \prod_{e \in \Sigma_{1}} L_{f}\left(\chi_{e}, \varphi(e)\right)
$$

By the preceding lemma, we see that $\hat{H}_{\Sigma}(\cdot, \varphi)$ is absolutely integrable over $\mathcal{A}_{T}$. For $t \in T(\mathbf{A})$ we have

$$
\int_{T\left(\mathbf{A}_{F}\right)} H_{\Sigma}(x t, \varphi) \chi(x) d x=\chi^{-1}(t) \hat{H}_{\Sigma}(\chi, \varphi) .
$$

Hence we can apply Poisson's summation formula (together with (9)) and obtain

$$
\sum_{x \in T(F)} H_{\Sigma}(x t, \varphi)=\mu_{T} \int_{M_{\mathbf{R}}}\left\{\sum_{\chi \in \mathcal{U}_{T}} \hat{H}_{\Sigma}(\chi, \varphi+i m)\left(\chi \chi^{m}(t)\right)^{-1}\right\} d m,
$$

where the Lebesgue measure $d m$ on $M_{\mathbf{R}}$ is normalized by $M$ and

$$
\mu_{T}=\frac{1}{(2 \pi \kappa)^{d}}, \quad \kappa=\frac{\mathrm{cl}_{F} \cdot \mathrm{R}_{F}}{\mathrm{w}_{F}}
$$

with $\mathrm{cl}_{F}$ the class number, $\mathrm{R}_{F}$ the regulator and $\mathrm{w}_{F}$ the number of roots of unity in $F$. Note that $\hat{H}_{\Sigma}\left(\chi \cdot \chi^{m}, \varphi\right)=\hat{H}_{\Sigma}(\chi, \varphi+i m)$.

3.6 In section 5 we need uniform estimates for $L$-functions in a neighborhood of the line $\operatorname{Re}(s)=1$. For any unramified character $\chi: \mathbf{G}_{m}(\mathbf{A}) / \mathbf{G}_{m}(F) \rightarrow S^{1}$ and any archimedean place $v$ there exists a $\tau_{v} \in \mathbf{R}$ such that $\chi_{v}\left(x_{v}\right)=\left|x_{v}\right|_{v}^{i \tau_{v}}$ for all $x_{v} \in \mathbf{G}_{m}\left(F_{v}\right)$. We put

$$
\chi_{\infty}=\left(\tau_{v}\right)_{v \mid \infty} \in \mathbf{R}^{\operatorname{Val}_{\infty}(F)} \quad \text { and } \quad\left\|\chi_{\infty}\right\|=\max _{v \mid \infty}\left|\tau_{v}\right| .
$$


We will use the following theorem of Rademacher ([34], Theorems 4,5), which rests on the Phragmén-Lindelöf principle.

Theorem 3.6 For any $\epsilon>0$ there exists $a \delta>0$ and a constant $c(\epsilon)>0$ such that for all $s$ with $\operatorname{Re}(s)>1-\delta$ and all unramified Hecke characters which are non-trivial on $\mathbf{G}_{m}(\mathbf{A})^{1}$ one has

$$
\left|L_{f}(\chi, s)\right| \leq c(\epsilon)\left(1+|\operatorname{Im}(s)|+\left\|\chi_{\infty}\right\|\right)^{\epsilon} .
$$

For the trivial character $\chi=1$ one has

$$
\left|L_{f}(1, s)\right| \leq c(\epsilon)\left|\frac{1+s}{1-s}\right|(1+|\operatorname{Im}(s)|)^{\epsilon} .
$$

\section{Twisted products}

4.1 Let $G, P, W=P \backslash G$, etc. be as in section 2 and $T, \Sigma, X=X_{\Sigma}$, etc. be as in section 3. Let $\eta: P \rightarrow T$ be a homomorphism. Then $P$ acts from the right on $X \times G$ by

$$
(x, g) \cdot p:=\left(x \eta(p), p^{-1} g\right) .
$$

Since $\pi_{W}: G \rightarrow W$ is locally trivial, the quotient

$$
Y=X^{P} \times G:=(X \times G) / P
$$

exists as a variety over $F$. Moreover, the projection $X \times G \rightarrow G$ induces a morphism $\pi: Y \rightarrow W$ and $Y$ becomes a locally trivial fiber bundle over $W$ with fiber $X$ (compare [19], I.5.16). Hence, by the properties of $X$ (non-singular, projective), we see that $Y$ is a non-singular projective variety over $F$ (since we don't need the projectivity of $Y$ we will omit the short argument). The quotient morphism $X \times G \rightarrow Y$ will be denoted by $\pi_{Y}$. Let $\varphi \in P L(\Sigma)$ and let $L_{\varphi}$ be the line bundle defined in section 3.1. Recall that $L_{\varphi}$ is equipped with a canonical $T$-action $L_{\varphi} \times T \rightarrow L_{\varphi}$ compatible with the action of $T$ on $X$. Thus $P$ acts on $L_{\varphi} \times G$ by

$$
(l, g) \cdot p:=\left(l \cdot \eta(p), p^{-1} g\right)
$$


and we can define

$$
L_{\varphi}^{Y}=L_{\varphi}{ }^{P} \times G:=\left(L_{\varphi} \times G\right) / P .
$$

This is a line bundle over $Y$. We denote the class of a point $(l, g) \in L_{\varphi} \times G$ in $L_{\varphi}^{Y}$ by $[l, g]$. Note that $L_{\varphi}^{Y}$ (and even its isomorphism class in $\operatorname{Pic}(Y)$ ) depends on the fixed $T$-linearization $\theta_{-\varphi}$. In fact, for $\varphi \in P L(\Sigma)$ and $m \in M$ we have

$$
L_{\varphi+m}^{Y} \simeq L_{\varphi}^{Y} \otimes \pi^{*} L_{m \circ \eta} .
$$

Embedding $M$ in $P L(\Sigma) \oplus X^{*}(P)$ by $m \mapsto(m,-m \circ \eta)$ we see that $M$ is contained in the kernel of the homomorphism

$$
\begin{aligned}
\psi: P L(\Sigma) \times X^{*}(P) & \rightarrow \operatorname{Pic}(Y), \\
(\varphi, \lambda) & \mapsto \text { isomorphism class of } L_{\varphi}^{Y} \otimes \pi^{*} L_{\lambda} .
\end{aligned}
$$

4.2 In the following proposition we collect all relevant facts about the geometry of twisted products which we will need in the sequel.

Proposition 4.2 a) The sequence

$$
0 \rightarrow M \rightarrow P L(\Sigma) \oplus X^{*}(P) \rightarrow \operatorname{Pic}(Y) \rightarrow 0
$$

is exact.

b) The cone of effective divisors $\Lambda_{\mathrm{eff}}(Y) \subset \operatorname{Pic}(Y)_{\mathbf{R}}$ is the image of the closure of

$$
P L(\Sigma)^{+} \times X^{*}(P)^{+} \subset P L(\Sigma)_{\mathbf{R}} \oplus X^{*}(P)_{\mathbf{R}} .
$$

c) The anti-canonical line bundle $\omega_{Y}^{\vee}$ is isomorphic to $L_{\varphi_{\Sigma}}^{Y} \otimes \pi^{*} L_{2 \rho_{P}}$.

Proof. a) By [37], Proposition 6.10, there is an exact sequence

$$
F[X \times G]^{*} / F^{*} \rightarrow X^{*}(P) \rightarrow \operatorname{Pic}(Y) \rightarrow \operatorname{Pic}(X \times G) .
$$

Denote by $\pi_{X}: X \times G \rightarrow X$ the canonical projection. Let $L$ be an invertible sheaf on $Y$. Then

$$
\pi_{Y}^{*} L \simeq \pi_{X}^{*} L_{\varphi}
$$

(for some $\varphi \in P L(\Sigma)$ ) because $\operatorname{Pic}(X \times G)=\operatorname{Pic}(X) \oplus \operatorname{Pic}(G)=\operatorname{Pic}(X)$ (cf. [37], Lemme 6.6 (i) and Lemme 6.9 (iv)). Note that $\pi_{Y}^{*} L_{\varphi}^{Y} \simeq \pi_{X}^{*} L_{\varphi}$, so that

$$
\pi_{Y}^{*}\left(L \otimes L_{-\varphi}^{Y}\right)
$$


is trivial. Hence there exists a character $\lambda$ of $P$ such that $L \otimes L_{-\varphi}^{Y}$ is isomorphic to $\pi^{*} L_{\lambda}$ (the map $X^{*}(P) \rightarrow \operatorname{Pic}(Y)$ factorizes $X^{*}(P) \rightarrow \operatorname{Pic}(W) \rightarrow \operatorname{Pic}(Y)$ ). This shows surjectivity.

Suppose now that for $\varphi \in P L(\Sigma)$ and $\lambda \in X^{*}(P)$ the sheaf $L_{\varphi}^{Y} \otimes \pi^{*} L_{\lambda}$ is trivial on $Y$. Then $\pi_{Y}^{*}\left(L_{\varphi}^{Y} \otimes \pi^{*} L_{\lambda}\right) \simeq \pi_{X}^{*} L_{\varphi}$ is trivial on $X \times G$, therefore $L_{\varphi} \simeq \mathcal{O}_{X}, \varphi=m \in M$ and $L_{\varphi}^{Y} \otimes \pi^{*} L_{\lambda}=\pi^{*} L_{\lambda+m \circ \eta}$. By Rosenlicht's theorem,

$$
F[X \times G]^{*} / F^{*}=F[X]^{*} / F^{*} \oplus F[G]^{*} / F^{*}=X^{*}(G)=0,
$$

therefore, the map $X^{*}(P) \rightarrow \operatorname{Pic}(Y)$ is injective, hence $\lambda+m \circ \eta=0$ and $(\varphi, \lambda)$ is in the image of $M \rightarrow P L(\Sigma) \oplus X^{*}(P)$.

b) For $\varphi \in P L(\Sigma)$ denote by $\square_{\varphi}$ the set of all $m \in M$ such that for all $n \in N_{\mathbf{R}}$

$$
\varphi(n)+n(m) \geq 0 .
$$

By [30], Lemma 2.3, $\square_{\varphi}$ is a basis for $H^{0}\left(X, L_{\varphi}\right)$ (note the different sign conventions). It is easy to see that

$$
\pi_{*} L_{\varphi}^{Y} \simeq \oplus_{m \in \square_{\varphi}} L_{-m \circ \eta}
$$

Suppose $L_{\varphi}^{Y} \otimes \pi^{*} L_{\lambda}$ has a non-zero global section. Then

$$
\pi_{*}\left(L_{\varphi}^{Y} \otimes \pi^{*} L_{\lambda}\right) \simeq \oplus_{m \in \square_{\varphi}} L_{-m \circ \eta+\lambda}
$$

has a non-zero global section, hence (cf. section 2.3) there is a $m^{\prime} \in \square_{\varphi}$ such that $-m^{\prime} \circ \eta+\lambda$ is contained in the closure of $X^{*}(P)^{+}$. Putting $\lambda^{\prime}=-m^{\prime} \circ \eta+\lambda, \varphi^{\prime}=\varphi+m^{\prime}$ we have $L_{\varphi^{\prime}}^{Y} \otimes \pi^{*} L_{\lambda^{\prime}} \simeq L_{\varphi}^{Y} \otimes \pi^{*} L_{\lambda}$ and $\left(\varphi^{\prime}, \lambda^{\prime}\right)$ is contained in the closure of $P L(\Sigma)^{+} \times X^{*}(P)^{+}$.

On the other hand, if $(\varphi, \lambda) \in P L(\Sigma) \oplus X^{*}(P)$ is contained in the closure of $P L(\Sigma)^{+} \times$ $X^{*}(P)^{+}$, then the trivial character corresponds to a global section of $L_{\varphi}$. Hence

$$
\pi_{*}\left(L_{\varphi}^{Y} \otimes \pi^{*} L_{\lambda}\right)=L_{\lambda} \oplus \bigoplus_{m \in \square_{\varphi}-\{0\}} L_{-m \circ \eta+\lambda}
$$

and $H^{0}\left(W, L_{\lambda}\right) \neq\{0\}$, i.e., $L_{\varphi}^{Y} \otimes \pi^{*} L_{\lambda}$ has a non-zero global section.

c) Note first that the exact sequence

$$
0 \rightarrow \pi^{*} \Omega_{W} \rightarrow \Omega_{Y} \rightarrow \Omega_{Y / W} \rightarrow 0
$$

splits, and therefore $\omega_{Y} \simeq\left(\Lambda^{d} \Omega_{Y / W}\right) \otimes \pi^{*} \omega_{W}$. Since $\omega_{W} \simeq L_{-2 \rho_{P}}$ it remains to show that $\left(\Lambda^{d} \Omega_{Y / W}\right)^{\vee} \simeq L_{\varphi_{\Sigma}}^{Y}$. Let $\mathcal{J}_{Y / W}$ be the ideal sheaf of the image of the diagonal morphism

$$
\Delta_{Y / W}: Y \rightarrow Y \times_{W} Y .
$$


Now $Y \times{ }_{W} Y$ is canonically isomorphic to $(X \times X){ }^{P_{\times}} G$ and $\Delta_{Y / W}(Y)$ is just $\Delta_{X}(X){ }^{P_{\times}}$ $G$. Hence we see that $\left(\mathcal{J}_{Y / W} / \mathcal{J}_{Y / W}^{2}\right)^{\vee}$ is the sheaf of local sections of

$$
\mathbf{V}\left(\mathcal{J}_{X} / \mathcal{J}_{X}^{2}\right)^{P} \times G
$$

where $\mathcal{J}_{X}$ is the ideal sheaf of $\Delta_{X}(X) \subset X \times X$ and $\mathbf{V}$ denotes the associated geometric vector bundle (with the same convention as in [17], II, Exercise 5.18). Pulling back to $Y$ and taking the $d$-th exterior power, we get

$$
\mathbf{V}\left(\Lambda^{d} \Omega_{Y / W}\right) \simeq \mathbf{V}\left(\Lambda^{d} \Omega_{X}\right){ }^{P} \times G \simeq \mathbf{V}\left(\omega_{X}\right)^{P_{\times}} G
$$

The canonical $T$-linearization of $\omega_{X}$ (induced by the action of $T$ on rational functions) corresponds to the $T$-linearization $\theta_{-\varphi_{\Sigma}}$ of $L_{-\varphi_{\Sigma}} \simeq \omega_{X}$, i.e.,

$$
\mathbf{L}_{\varphi_{\Sigma}} P_{\times}=\mathbf{V}\left(\omega_{X}\right)^{P} \times G
$$

and we get $L_{\varphi_{\Sigma}}^{Y} \simeq\left(\Lambda^{d} \Omega_{Y / W}\right)^{\vee}$.

4.3 We are going to introduce an adelic metric on the line bundles $L_{\varphi}^{Y}$. Let $v$ be a valuation of $F$ and let $y=\pi_{W}(x, g) \in Y\left(F_{v}\right)$ with $(x, g) \in X\left(F_{v}\right) \times G\left(F_{v}\right)$. Any point in $L_{\varphi}^{Y}\left(F_{v}\right)$ lying aver $y$ may be written in the form $[l, g]$ with $l$ a point of $L_{\varphi}\left(F_{v}\right)$ lying over $x$. Then we put

$$
\|[l, g]\|_{y}:=\|l \cdot \eta(p)\|_{x \cdot \eta(p)}
$$

where $g=p k, p \in P\left(F_{v}\right), k \in \mathbf{K}_{G, v}$ and the norm on the right is the one defined in section 3.2 .

Since the $v$-adic metric on $L_{\varphi}$ is invariant under $\mathbf{K}_{T, v}$ this is well-defined. The family $\|\cdot\|_{v}=(\|\cdot\|)_{y \in Y\left(F_{v}\right)}$ is a $v$-adic metric on $L_{\varphi}^{Y}$ and $\mathcal{L}_{\varphi}^{Y}=\left(L_{\varphi}^{Y},\left(\|\cdot\|_{v}\right)_{v}\right)$ is a metrization of $L_{\varphi}^{Y}$. Let

$$
Y^{o}=T^{P} \times G \hookrightarrow X^{P} \times G=Y
$$

be the twisted product of $T$ with $W$. Over $Y^{o}$ there is a canonical section of $L_{\varphi}^{Y}$, namely

$$
\begin{array}{ccc}
s_{\varphi}: \quad Y^{o} & \rightarrow \mathbf{L}_{\varphi}^{Y}, \\
& \pi_{Y}(x, g) & \mapsto\left[\left[x, m_{\varphi, \sigma}(x)\right], g\right],
\end{array}
$$

where we consider $\left(x, m_{\varphi, \sigma}(x)\right)$ as an element of $U_{\sigma} \times \mathbb{A}^{1}$ (cf. the definition of $\left.L_{\varphi}\right)$. 
Then, for $y=\pi_{Y}(x, g) \in Y^{o}\left(F_{v}\right)$ and $g=p k$ (Iwasawa decomposition) we have

$$
\begin{aligned}
\left\|s_{\varphi}(y)\right\|_{y} & =\left\|\left[x \eta(p), m_{\varphi, \sigma}(\eta(p)) \cdot m_{\varphi, \sigma}(x)\right]\right\|_{x \eta(p)} \\
& =H_{\Sigma, v}(x \eta(p), \varphi)
\end{aligned}
$$

(cf. section 3.2). Globally, for $y=\pi_{Y}(x, \gamma) \in Y^{o}(F), x \in T(F), \gamma=p_{\gamma} k_{\gamma} \in G(F)$, $p_{\gamma} \in P(\mathbf{A}), k_{\gamma} \in \mathbf{K}_{G}$, we get

$$
H_{\mathcal{L}_{\varphi}^{Y}}(y)=\prod_{v}\left\|s_{\varphi}\left(y_{v}\right)\right\|_{y_{v}}^{-1}=H_{\Sigma}\left(x \eta\left(p_{\gamma}\right),-\varphi\right) .
$$

4.4 Let $\xi: P(\mathbf{A}) / P(F) \rightarrow S^{1}$ be an unramified character, i.e., $\xi$ is trivial on $P(\mathbf{A}) \cap \mathbf{K}_{G}$. Using the Iwasawa decomposition we get a well defined function

$$
\begin{gathered}
\phi_{\xi}: G(\mathbf{A}) \rightarrow S^{1}, \\
\phi_{\xi}(g)=\xi(p),
\end{gathered}
$$

if $g=p k$ as above. We denote by

$$
E_{P}^{G}(\lambda, \xi, g)=\sum_{\gamma \in P(F) \backslash G(F)} \phi_{\xi}(\gamma g) e^{\left\langle\lambda+\rho_{P}, H_{P}(\gamma g)\right\rangle}
$$

the corresponding Eisenstein series and we put $E_{P}^{G}(\lambda, \xi)=E_{P}^{G}\left(\lambda, \xi, 1_{G}\right)$. This series converges absolutely for $\operatorname{Re}(\lambda)$ contained in the cone $\rho_{P}+X^{*}(P)^{+}$(cf. (2.3)). A character $\chi \in \mathcal{A}_{T}$ induces a character $\chi_{\eta}=\chi \circ \eta: P(\mathbf{A}) / P(F) \rightarrow S^{1}$. We denote by $\check{\eta}: X^{*}(T)_{\mathbf{R}} \rightarrow$ $X^{*}(P)_{\mathbf{R}}$ the map on characters induced by $\eta$.

Proposition 4.4 Let $L$ be a line bundle on $Y$ such that its class is contained in the interior of the cone $\Lambda_{\mathrm{eff}}(Y)$. Let $(\varphi, \lambda)$ be in $P L(\Sigma)^{+} \times X^{*}(P)^{+}$with $\psi(\varphi, \lambda)=[L]$. There is a metrization $\mathcal{L}$ of $L$ such that for all s with $\operatorname{Re}(s)(\varphi, \lambda) \in\left(\varphi_{\Sigma}, 2 \rho_{P}\right)+P L(\Sigma)^{+} \times X^{*}(P)^{+}$ the series

$$
Z_{Y^{o}}(\mathcal{L}, s)=\sum_{y \in Y^{o}(F)} H_{\mathcal{L}}(y)^{-s}
$$

converges absolutely. Moreover, for these $s$

$$
Z_{Y^{o}}(\mathcal{L}, s)=\mu_{T} \int_{M_{\mathbf{R}}}\left\{\sum_{\chi \in \mathcal{U}_{T}} \hat{H}_{\Sigma}(\chi, s \varphi+i m) E_{P}^{G}\left(s \lambda-\rho_{P}-i \check{\eta}(m), \chi_{\eta}^{-1}\right)\right\} d m
$$


where the sum and integral on the right converge absolutely as well.

Proof. Let $\left(\varphi^{\prime}, \lambda^{\prime}\right) \in P L(\Sigma) \oplus X^{*}(P)$ such that there is an isomorphism $L \simeq L_{\varphi^{\prime}}^{Y} \oplus \pi^{*} L_{\lambda^{\prime}}$. Denote by $\mathcal{L}$ the metrization of $L$ which is the pullback of $\mathcal{L}_{\varphi^{\prime}}^{Y} \otimes \pi^{*} \mathcal{L}_{\lambda^{\prime}}$ via this isomorphism. Let $m \in M_{\mathbf{R}}$ such that

$$
(\varphi, \lambda)=\left(\varphi^{\prime}+m,-\check{\eta}(m)+\lambda^{\prime}\right)
$$

is contained in $P L(\Sigma)^{+} \times X^{*}(P)^{+}$. We have for any $y \in Y^{o}(F), y=\pi_{Y}(x, \gamma)$ with $x \in T(F), \gamma \in G(F)$ and $\gamma=p_{\gamma} k_{\gamma}$

$$
\begin{aligned}
H_{\mathcal{L}}(y) & =H_{\mathcal{L}_{\varphi^{\prime}}^{Y} \otimes \pi^{*} \mathcal{L}_{\lambda^{\prime}}}(y)=e^{-\left\langle\lambda^{\prime}, H_{P}(\gamma)\right\rangle} H_{\Sigma}\left(x \eta\left(p_{\gamma}\right),-\varphi^{\prime}\right) \\
& =e^{-\left\langle\lambda^{\prime}-m \circ \eta, H_{P}(\gamma)\right\rangle} H_{\Sigma}\left(x \eta\left(p_{\gamma}\right),-\left(\varphi^{\prime}+m\right)\right)=e^{-\left\langle\lambda, H_{P}(\gamma)\right\rangle} H_{\Sigma}\left(x \eta\left(p_{\gamma}\right),-\varphi\right) .
\end{aligned}
$$

We consider $s=u+i v \in \mathbf{C}$ such that $u \cdot \varphi$ is contained in the shifted cone $\varphi_{\Sigma}+P L(\Sigma)^{+}$ and $u \cdot \lambda$ is contained in the cone $2 \rho_{P}+X^{*}(P)^{+}$. Then

$$
\sum_{x \in T(F)} H_{\Sigma}\left(x \eta\left(p_{\gamma}\right), u \varphi\right)
$$

converges by Proposition 3.4 and is equal to

$$
\mu_{T} \int_{M_{\mathbf{R}}}\left\{\sum_{\chi \in \mathcal{U}_{T}} \hat{H}\left(\chi \chi^{m}, u \varphi\right) \chi \chi^{m}\left(\eta\left(p_{\gamma}\right)\right)^{-1}\right\} d m
$$

(cf. (13)). Moreover, $\hat{H}_{\Sigma}(\cdot, u \varphi)$ is absolutely convergent on $\mathcal{A}_{T}$ and therefore

$$
\sum_{x \in T(F)} H_{\Sigma}\left(x \eta\left(p_{\gamma}\right), u \varphi\right) \leq \mu_{T} \int_{M_{\mathbf{R}}}\left\{\sum_{\chi \in \mathcal{U}_{T}}\left|\hat{H}_{\Sigma}\left(\chi \chi^{m}, u \varphi\right)\right|\right\} d m
$$

is bounded by some constant $c$ (which is independent of $\eta\left(p_{\gamma}\right)$ ). Thus we may calculate

$$
\begin{aligned}
\sum_{y \in Y^{o}(F)}\left|H_{\mathcal{L}}(y)^{-s}\right| & =\sum_{\gamma \in P(F) \backslash G(F)} e^{\left\langle u \lambda, H_{P}(\gamma)\right\rangle} \sum_{x \in T(F)} H_{\Sigma}\left(x \eta\left(p_{\gamma}\right), u \varphi\right) \\
& \leq c \sum_{\gamma \in P(F) \backslash G(F)} e^{\left\langle u \lambda, H_{P}(\gamma)\right\rangle} .
\end{aligned}
$$

This shows the first assertion. Since

$$
\mu_{T} \int_{M_{\mathbf{R}}}\left\{\sum_{\chi \in \mathcal{U}_{T}}\left|\hat{H}_{\Sigma}\left(\chi \chi^{m}, u \varphi\right)\right|\right\} d m
$$


converges, we can interchange the summation and integration and get

$$
\begin{aligned}
Z_{Y^{o}}(\mathcal{L}, s) & =\sum_{\gamma \in P(F) \backslash G(F)} e^{\left\langle s \lambda, H_{P}(\gamma)\right\rangle} \mu_{T} \int_{M_{\mathbf{R}}}\left\{\sum_{\chi \in \mathcal{U}_{T}} \hat{H}_{\Sigma}\left(\chi \chi^{m}, s \varphi\right)\left(\chi \chi^{m}\right)^{-1}\left(\eta\left(p_{\gamma}\right)\right)\right\} d m \\
& =\mu_{T} \int_{M_{\mathbf{R}}}\left\{\sum_{\chi \in \mathcal{U}_{T}} \hat{H}_{\Sigma}(\chi, s \varphi+i m) E_{P}^{G}\left(s \lambda-\rho_{P}-i \check{\eta}(m), \chi_{\eta}^{-1}\right)\right\} d m .
\end{aligned}
$$

\section{Meromorphic continuation}

5.1 The proposition in section 4.4 gives an expression of the height zeta function (for the open subset $Y^{o} \subset Y$ ) which we will use to determine the asymptotic behavior of the counting function $N_{Y^{o}}(\mathcal{L}, H)$ (cf. sec. 1) by applying a Tauberian theorem.

The first thing to do is to show that $Z_{Y^{o}}(\mathcal{L}, s)$ can be continued meromorphically to a halfspace beyond the abscissa of convergence and that there is no pole on this line with non-zero imaginary part. Then it remains to prove that this abscissa is at $\operatorname{Re}(s)=a(L)$ and to determine the order of the pole in $s=a(L)$. We will see that this order is $b(L)$.

The method which we will explain now consists in an iterated application of Cauchy's residue theorem. The proofs will be given in section 7 .

5.2 Let $E$ be a finite dimensional vector space over $\mathbf{R}$ and $E_{\mathbf{C}}$ its complexification. Let $V \subset E$ be a subspace and let $l_{1}, \ldots, l_{m} \in E^{\vee}=\operatorname{Hom}_{\mathbf{R}}(E, \mathbf{R})$ be linearly independent linear forms. Put $H_{j}=\operatorname{Ker}\left(l_{j}\right)$ for $j=1, \ldots, m$.

Let $B \subset E$ be an open and convex neighborhood of $\mathbf{0}$ such that for all $x \in B$ and $j=1, \ldots, m$ we have $l_{j}(x)>-1$. Let $T_{B}=B+i E \subset E_{\mathbf{C}}$ be the tube domain over $B$ and denote by $\mathcal{M}\left(T_{B}\right)$ the set of meromorphic functions on $T_{B}$. We consider meromorphic functions $f \in \mathcal{M}\left(T_{B}\right)$ with the following properties: The function

$$
g(z)=f(z) \prod_{j=1}^{m} \frac{l_{j}(z)}{l_{j}(z)+1}
$$


is holomorphic in $T_{B}$ and there is a sufficient function $c: V \rightarrow \mathbf{R}_{\geq 0}$ such that for all compacts $\mathbf{K} \in T_{B}$, all $z \in \mathbf{K}$ and all $v \in V$, we have the estimate

$$
|g(z+i v)| \leq \kappa(\mathbf{K}) c(v)
$$

(Cf. section 7.3 for a precise definition of a sufficient function. In particular, such a sufficient function is absolutely integrable over any subspace $U \subset V$.) In this case we call $f$ distinguished with respect to the data $\left(V ; l_{1}, \ldots, l_{m}\right)$.

Let $C$ be a connected component of $B-\cup_{j=1}^{m} H_{j}$. By the conditions on $g$ the integral

$$
\tilde{f}_{C}(z):=\frac{1}{(2 \pi)^{\nu}} \int_{V} f(z+i v) d v
$$

$\left(\nu=\operatorname{dim} V\right.$ and $d v$ is a fixed Lebesgue measure on $V$ ) converges for every $z \in T_{C}$ and $\tilde{f}_{C}$ is a holomorphic function on $T_{C}$.

Theorem 5.2 There is an open neighborhood $\tilde{B}$ containing $C$, and linear forms $\tilde{l}_{1}, \ldots, \tilde{l}_{\tilde{m}}$ which vanish on $V$ such that

$$
z \mapsto \tilde{f}_{C}(z) \prod_{j=1}^{\tilde{m}} \tilde{l}_{j}(z)
$$

has a holomorphic continuation to $T_{\tilde{B}}$. Moreover, for all $j \in\{1, \ldots, \tilde{m}\}$ we have $\operatorname{Ker}\left(\tilde{l}_{j}\right) \cap$ $C=\emptyset$.

We shall give the proof of this theorem in sections 7.3 and 7.4 .

5.3 Put $E^{(0)}=\cap_{j=1}^{m} \operatorname{Ker}\left(l_{j}\right)$ and $E_{0}=E / E^{(0)}$. Let $\pi_{0}: E \rightarrow E_{0}$ be the canonical projection and suppose $V \cap E^{(0)}=\{\mathbf{0}\}$. Let

$$
E_{0}^{+}=\left\{x \in E_{0} \mid l_{j}(x) \geq 0 \text { for all } j=1, \ldots, m\right\}
$$

and let $\psi_{0}: E_{0} \rightarrow P:=E_{0}^{+} / \pi_{0}(V)$ be the canonical projection. We want to assume that $\pi_{0}(V) \cap E_{0}^{+}=\{\mathbf{0}\}$, so that $\Lambda:=\psi_{0}\left(E_{0}^{+}\right)$is a strictly convex polyhedral cone. Let $d y$ be the Lebesgue measure on $E_{0}^{\vee}$ normalized by the lattice $\oplus_{j=1}^{m} \mathbf{Z} l_{j}$. Let $A \subset V$ be a lattice and let $d v$ be the measure on $V$ normalized by $A$. On $V^{\vee}$ we have the Lebesgue measure $d y^{\prime}$ normalized by $A^{\vee}$ and a section of the projection $E_{0}^{\vee} \rightarrow V^{\vee}$ gives a measure $d y^{\prime \prime}$ on $P^{\vee}$ with $d y=d y^{\prime} d y^{\prime \prime}$. 
Define the $\mathcal{X}$-function of the cone $\Lambda$ by

$$
\mathcal{X}_{\Lambda}(x)=\int_{\Lambda^{\vee}} e^{-y^{\prime \prime}(x)} d y^{\prime \prime}
$$

for all $x \in P_{\mathbf{C}}$ with $\operatorname{Re}(x)$ contained in the interior of $\Lambda$ (cf. section 7.1).

Let $B \subset E$ be as above and let $f \in \mathcal{M}\left(T_{B}\right)$ be a distinguished function with respect to $\left(V ; l_{1}, \ldots, l_{m}\right)$. Put

$$
\begin{gathered}
g(z)=f(z) \prod_{j=1}^{m} \frac{l_{j}(z)}{l_{j}(z)+1}, \\
B^{+}=B \cap\left\{x \in E \mid l_{j}(x)>0, \text { for all } j=1, \ldots, m\right\}, \\
\tilde{f}_{B^{+}}(z)=\frac{1}{(2 \pi)^{\nu}} \int_{V} f(z+i v) d v
\end{gathered}
$$

(for $z \in T_{B}$ ). The function $\tilde{f}_{B^{+}}: T_{B^{+}} \rightarrow \mathbf{C}$ is holomorphic and has a meromorphic continuation to a neighborhood of $\mathbf{0} \in E_{\mathbf{C}}$. In section 7.5 we will prove the following theorem.

Theorem 5.3 For $x_{0} \in B^{+}$we have

$$
\lim _{s \rightarrow 0} s^{m-\nu} \tilde{f}_{B^{+}}\left(s x_{0}\right)=g(\mathbf{0}) \mathcal{X}_{\Lambda}\left(\psi_{0}\left(x_{0}\right)\right) .
$$

5.4 In this section we make some preparations in order to apply the general setting of 5.2. Let $L$ be a line bundle on $Y$ such that its class in $\operatorname{Pic}(Y)$ lies in the interior of $\Lambda_{\text {eff }}(Y)$. By the definition of $a(L)$ (cf. section 1 and Proposition 4.2),

$$
a(L)[L]-\psi\left(\varphi_{\Sigma}, 2 \rho_{P}\right) \in \Lambda(L)
$$

where $\Lambda(L)$ is the minimal face of $\Lambda_{\text {eff }}(Y)$ containing $a(L)[L]-\psi\left(\varphi_{\Sigma}, 2 \rho_{P}\right)$. Define $\varphi_{e} \in$ $P L(\Sigma)$ (for $e \in \Sigma_{1}$ ) by $\varphi_{e}\left(e^{\prime}\right)=\delta_{e e^{\prime}}$, for all $e^{\prime} \in \Sigma_{1}$ and put

$$
\Sigma_{1}^{\prime}:=\left\{e \in \Sigma_{1} \mid \psi\left(\varphi_{e}, \mathbf{0}\right) \in \Lambda(L)\right\} .
$$

Let $P^{\prime} \subset G$ be the standard parabolic subgroup with

$$
\Delta_{P^{\prime}}=\left\{\alpha \in \Delta_{P} \mid \psi\left(\mathbf{0}, \varpi_{\alpha}\right) \in \Lambda(L)\right\},
$$


where $\left\langle\varpi_{\alpha}, \beta\right\rangle=\delta_{\alpha \beta}$ for all $\alpha, \beta \in \Delta_{0}$. Let

$$
\left(\varphi_{L}, \lambda_{L}\right) \in\left(\sum_{e \in \Sigma_{1}^{\prime}} \mathbf{R}_{>0} \varphi_{e}\right) \times\left(\sum_{\alpha \in \Delta_{P^{\prime}}} \mathbf{R}_{>0} \varpi_{\alpha}\right)
$$

such that $\psi\left(\varphi_{L}, \lambda_{L}\right)=a(L)[L]-\psi\left(\varphi_{\Sigma}, 2 \rho_{P}\right)$. Then

$$
\hat{L}:=\frac{1}{a(L)}\left(\varphi_{\Sigma}+\varphi_{L}, 2 \rho_{P}+\lambda_{L}\right)
$$

is mapped onto $[L]$ by $\psi$. Denote by

$$
h_{L}(\varphi, \lambda):=\prod_{e \in \Sigma_{1}-\Sigma_{1}^{\prime}} \frac{\varphi(e)}{\varphi(e)+1} \prod_{\alpha \in \Delta_{P}-\Delta_{P^{\prime}}} \frac{\langle\lambda, \alpha\rangle}{\langle\lambda, \alpha\rangle+1}
$$

and put

$$
\tilde{\varphi}=\varphi+\varphi_{\Sigma}+\varphi_{L} \quad \text { and } \quad \tilde{\lambda}=\lambda+\rho_{P}+\lambda_{L}
$$

From now on we will denote by $\mathbf{K}_{G} \subset G(\mathbf{A})$ the maximal compact subgroup defined in section 8.2 .

Lemma 5.4 There exists a convex open neighborhood $B$ of $\mathbf{0}$ in $P L(\Sigma)_{\mathbf{R}} \oplus X^{*}(P)_{\mathbf{R}}$ with the following property: For any compact subset $\mathbf{K} \subset T_{B}$ there is a constant $c=c(\mathbf{K})>0$ such that for all $(\varphi, \lambda) \in \mathbf{K}, \chi \in \mathcal{U}_{T}$ and $m \in M_{\mathbf{R}}$ we have

$$
\begin{aligned}
& \left|\hat{H}_{\Sigma}(\chi, \tilde{\varphi}+i m) E_{P}^{G}\left(\tilde{\lambda}-i \check{\eta}(m), \chi_{\eta}^{-1}\right) h_{L}(\varphi+i m, \lambda-i \check{\eta}(m))\right| \\
& \leq c \prod_{v \mid \infty}\left\{\sum_{\operatorname{dim} \sigma=d} \prod_{e \in \sigma \cap \Sigma_{1}} \frac{1}{\left(1+\left|e\left(m+m_{v}(\chi)\right)\right|\right)^{1+1 / 2 d}}\right\}
\end{aligned}
$$

Proof. Write as in (12)

$$
\hat{H}_{\Sigma}(\chi, \tilde{\varphi}+i m)=\zeta_{\Sigma}(\chi, \tilde{\varphi}+i m) \prod_{e \in \Sigma_{1}} L_{f}\left(\chi_{e}, 1+\left(\varphi+\varphi_{L}+i m\right)(e)\right) .
$$

For $\operatorname{Re}(\varphi)$ sufficiently small and $e \in \Sigma_{1}^{\prime}$ we have

$$
\operatorname{Re}(\varphi(e))+\varphi_{L}(e) \geq \frac{1}{2} \varphi_{L}(e)>0 .
$$


Hence

$$
\left|\prod_{e \in \Sigma_{1}^{\prime}} L_{f}\left(\chi_{e}, 1+\left(\varphi+\varphi_{L}+i m\right)(e)\right)\right|
$$

is bounded for $\operatorname{Re}(\varphi)$ sufficiently small. If $e \in \Sigma_{1}-\Sigma_{1}^{\prime}$, then $\varphi_{L}(e)=0$. By the estimates of Rademacher (cf. Theorem 3.6), we have for $\chi_{e} \neq 1$

$$
\left|L_{f}\left(\chi_{e}, 1+(\varphi+i m)(e)\right)\right| \leq c_{e}\left(1+|m(e)|+\left\|\left(\chi_{e}\right)_{\infty}\right\|\right)^{\epsilon}
$$

for $\operatorname{Re}(\varphi(e))>-\delta$ and $\varphi$ in a compact set ( $\delta$ depends on $\epsilon, c_{e}$ depends on this compact subset). If $\chi_{e}=1$ (abusing notations we will denote from now on the trivial character by 1 ), then

$$
\frac{(\varphi+i m)(e)}{(\varphi+i m)(e)+1}\left|L_{f}(1,1+(\varphi+i m)(e))\right| \leq c_{e}(1+|m(e)|)^{\epsilon} .
$$

Now we use Proposition 8.7 concerning estimates for Eisenstein series. This proposition tells us that there is for given $\epsilon>0$ an open neighborhood of $\mathbf{0}$ in $X^{*}(P)_{\mathbf{R}}$ such that for $\operatorname{Re}(\lambda)$ contained in this neighborhood

$\left|\prod_{\alpha \in \Delta_{P}} \frac{\left\langle\lambda+\lambda_{L}-i \check{\eta}(m), \alpha\right\rangle}{\left\langle\lambda+\lambda_{L}-i \check{\eta}(m), \alpha\right\rangle+1} E_{P}^{G}\left(\tilde{\lambda}-i \check{\eta}(m), \chi_{\eta}^{-1}\right)\right| \leq c_{1}\left(1+\|\operatorname{Im}(\lambda)+\check{\eta}(m)\|+\left\|\left(\chi_{\eta}{ }^{-1}\right)_{\infty}\right\|\right)^{\epsilon}$.

(For the definition of $(\cdots)_{\infty}$ and the norms see section 8.5.) If we let $\lambda$ vary in a compact subset in the tube domain over this neighborhood, then there is a constant $c_{2} \geq c_{1}$ such that

$$
c_{1}\left(1+\|\operatorname{Im}(\lambda)+\check{\eta}(m)\|+\left\|\left(\chi_{\eta}{ }^{-1}\right)_{\infty}\right\|\right)^{\epsilon} \leq c_{2}\left(1+\|\check{\eta}(m)\|+\left\|\left(\chi_{\eta}{ }^{-1}\right)_{\infty}\right\|\right)^{\epsilon} .
$$

For $\operatorname{Re}(\lambda)$ sufficiently small and $\alpha \in \Delta_{P^{\prime}}$ we have

$$
\left\langle\operatorname{Re}(\lambda)+\lambda_{L}, \alpha\right\rangle \geq \frac{1}{2}\left\langle\lambda_{L}, \alpha\right\rangle>0 .
$$

Therefore, there are $c_{3}, c_{4}>0$ such that for all such $\lambda$ and $m \in M_{\mathbf{R}}$ we have

$$
c_{3} \leq\left|\prod_{\alpha \in \Delta_{P^{\prime}}} \frac{\left\langle\lambda+\lambda_{L}-i \check{\eta}(m), \alpha\right\rangle}{\left\langle\lambda+\lambda_{L}-i \check{\eta}(m), \alpha\right\rangle+1}\right| \leq c_{4}
$$


Putting everything together, we can conclude that there is a neighborhood $B$ of $\mathbf{0}$ in $P L(\Sigma)_{\mathbf{R}} \oplus X^{*}(P)_{\mathbf{R}}$ such that for $(\varphi, \lambda)$ in a compact subset $\mathbf{K}$ of the tube domain over $B$ we have

$$
\begin{aligned}
& \left|\prod_{e \in \Sigma_{1}} L_{f}\left(\chi_{e}, 1+\left(\varphi+\varphi_{L}+i m\right)(e)\right) \prod_{e \in \Sigma_{1}-\Sigma_{1}^{\prime}} \frac{(\varphi+i m)(e)}{(\varphi+i m)(e)+1}\right| \\
& \quad \times\left|E_{P}^{G}\left(\tilde{\lambda}-i \check{\eta}(m), \chi_{\eta}^{-1}\right) \prod_{\alpha \in \Delta_{P}-\Delta_{P^{\prime}}} \frac{\langle\lambda-i \check{\eta}(m), \alpha\rangle}{\langle\lambda-i \check{\eta}(m), \alpha\rangle+1}\right| \leq c^{\prime}(\mathbf{K})\left(1+\left\|m+m_{\infty}(\chi)\right\|\right)^{\epsilon},
\end{aligned}
$$

where $\|\cdot\|$ is a norm on $M_{\mathbf{R}, \infty}$. On the other hand, by Lemma 3.5 , we have

$$
\left|\zeta_{\Sigma}(\chi, \tilde{\varphi}+i m)\right| \leq c^{\prime \prime}(\mathbf{K}) \prod_{v \mid \infty}\left\{\sum_{\operatorname{dim} \sigma=d} \prod_{e \in \sigma \cap \Sigma_{1}} \frac{1}{\left(1+\left|e\left(m+m_{v}(\chi)\right)\right|\right)^{1+1 / d}}\right\} .
$$

Now we may choose $\epsilon$ and $c_{5}$ such that

$$
\left(1+\left\|m+m_{\infty}(\chi)\right\|\right)^{\epsilon} \leq c_{5} \prod_{v \mid \infty} \prod_{e \in \sigma_{v} \cap \Sigma_{1}}\left(1+\left|e\left(m+m_{v}(\chi)\right)\right|\right)^{1 / 2 d}
$$

for any system $\left(\sigma_{v}\right)_{v \mid \infty}$ of $d$-dimensional cones. This gives the claimed estimate.

5.5 To begin with, we let

$$
\begin{gathered}
M_{\mathbf{R}}^{\prime}:=\left\{m \in M_{\mathbf{R}} \mid e(m)=0 \forall e \in \Sigma_{1}-\Sigma_{1}^{\prime} \text { and }\langle\check{\eta}(m), \alpha\rangle=0 \forall \alpha \in \Delta_{P}-\Delta_{P^{\prime}}\right\}, \\
M^{\prime}=M_{\mathbf{R}}^{\prime} \cap M .
\end{gathered}
$$

Then $M_{\mathbf{R}}^{\prime}=M^{\prime} \otimes \mathbf{R}$ and $M^{\prime \prime}=M / M^{\prime}$ is torsion free. Put $d^{\prime}=\operatorname{rank}\left(M^{\prime}\right), d^{\prime \prime}=\operatorname{rank}\left(M^{\prime \prime}\right)$.

The connection with sections 5.2 and 5.3 is as follows:

$$
\begin{aligned}
& E=\left(P L(\Sigma)_{\mathbf{R}} \oplus X^{*}(P)_{\mathbf{R}}\right) / M_{\mathbf{R}}^{\prime}, \\
& V=M^{\prime \prime} \otimes \mathbf{R}, A=M^{\prime \prime}, \nu=d^{\prime \prime},
\end{aligned}
$$

the set of linear forms $l_{1}, \ldots, l_{m}$ is given as

$$
(\varphi, \lambda)+M_{\mathbf{R}}^{\prime} \mapsto \varphi(e), e \in \Sigma_{1}-\Sigma_{1}^{\prime},
$$




$$
(\varphi, \lambda)+M_{\mathbf{R}}^{\prime} \mapsto\langle\lambda, \alpha\rangle, \alpha \in \Delta_{P}-\Delta_{P^{\prime}} .
$$

The measure $d v=d m^{\prime \prime}$ on $V=M_{\mathbf{R}}^{\prime \prime}$ is normalized by $M^{\prime \prime}, d m=d m^{\prime} d m^{\prime \prime}$, where $d m$ (resp. $d m^{\prime}$ ) is the Lebesgue measure on $M_{\mathbf{R}}$ (resp. $M_{\mathbf{R}}^{\prime}$ ) normalized by $M$ (resp. $M^{\prime}$ ).

Fix a convex open neighborhood of $\mathbf{0}$ in $P L(\Sigma)_{\mathbf{R}} \oplus X^{*}(P)_{\mathbf{R}}$ for which Lemma 5.4 is valid. Denote by $B$ the image of this neighborhood in $E$. This is an open and convex neighborhood of $\mathbf{0}$. Using Lemma 5.4 we see that

$$
g(\varphi, \lambda)=\mu_{T^{\prime}} \int_{M_{\mathrm{R}}^{\prime}} \frac{1}{\kappa^{d^{\prime \prime}}}\left\{\sum_{\chi \in \mathcal{U}_{T}} \hat{H}_{\Sigma}\left(\chi, \tilde{\varphi}+i m^{\prime}\right) E_{P}^{G}\left(\tilde{\lambda}-i \check{\eta}\left(m^{\prime}\right), \chi_{\eta}^{-1}\right) h_{L}(\varphi, \lambda)\right\} d m^{\prime}
$$

is a holomorphic function on $T_{B}$ (here $\mu_{T^{\prime}}=1 /(2 \pi \kappa)^{d^{\prime}}$ ). (We use the invariance of $g$ under $i M_{\mathbf{R}}^{\prime}$ and Cauchy-Riemann differential equations to check that $g$ is actually a function on $T_{B}$.) Hence,

$$
f(\varphi, \lambda):=g(\varphi, \lambda) h_{L}(\varphi, \lambda)^{-1}
$$

is a meromorphic function on $T_{B}$.

Let $\tilde{E}^{(0)}$ be the common kernel of all maps $(17,18)$ considered as a subspace of $P L(\Sigma)_{\mathbf{R}} \oplus X^{*}(P)_{\mathbf{R}}$. Note that there is an exact sequence

$$
0 \rightarrow M_{\mathbf{R}}^{\prime} \rightarrow \tilde{E}^{(0)} \rightarrow\langle\Lambda(L)\rangle \rightarrow 0
$$

which implies

$$
b(L)=\operatorname{codim} \Lambda(L)=m-d^{\prime \prime},
$$

where $m=\#\left(\Sigma_{1}-\Sigma_{1}^{\prime}\right)+\#\left(\Delta_{P}-\Delta_{P^{\prime}}\right)$.

Put $E^{(0)}=\tilde{E}^{(0)} / M_{\mathbf{R}}^{\prime} \subset E$. By construction, $M_{\mathbf{R}}^{\prime \prime} \cap E^{(0)}=\{\mathbf{0}\}$. Define as in section 5.3: $E_{0}=E / E^{(0)}$. Let $d y$ be the Lebesgue measure on $E_{0}^{\vee}$ normalized by the lattice generated by the linear forms $(17,18)$. Denote by $E_{0}^{+}$the closed simplicial cone in $E_{0}$ defined by these linear forms, and by $\pi_{0}: E \rightarrow E_{0}$ the canonical projection. It is easily seen that $\pi_{0}\left(M_{\mathbf{R}}^{\prime \prime}\right) \cap E_{0}^{+}=\{\mathbf{0}\}$ (using the exact sequence above). Let

$$
\psi_{0}: E_{0} \rightarrow P:=E_{0} / \pi_{0}\left(M_{\mathbf{R}}^{\prime \prime}\right)
$$

be the canonical projection and put

$$
\Lambda=\psi_{0}\left(E_{0}^{+}\right),
$$




$$
B^{+}=B \cap\left\{(\varphi, \lambda) \in E \mid \varphi(e)>0 \forall e \in \Sigma_{1}-\Sigma_{1}^{\prime},\langle\lambda, \alpha\rangle>0 \forall \alpha \in \Delta_{P}-\Delta_{P^{\prime}}\right\} .
$$

By the following theorem the function $f \in \mathcal{M}\left(T_{B}\right)$ is distinguished with respect to $M_{\mathbf{R}}^{\prime \prime}$ and the set of linear forms $(17,18)$. Therefore, we can define $\tilde{f}_{B^{+}}: T_{B^{+}} \rightarrow \mathbf{C}$ by

$$
\tilde{f}_{B^{+}}(z)=\frac{1}{(2 \pi)^{d^{\prime \prime}}} \int_{M_{\mathbf{R}}^{\prime \prime}} f\left(z+i\left(m^{\prime \prime},-\check{\eta}\left(m^{\prime \prime}\right)\right)\right) d m^{\prime \prime} .
$$

Theorem 5.5 $\quad$ a) $f$ is a distinguished function with respect to $M_{\mathbf{R}}^{\prime \prime}$ and the set of linear forms $(17,18)$.

b) There exist an open neighborhood $\tilde{B}$ of $\mathbf{0}$ containing $B^{+}$and linear forms $\tilde{l}_{1}, \ldots, \tilde{l}_{\tilde{m}}$ which vanish on $M_{\mathbf{R}}^{\prime \prime}$ such that

$$
\tilde{f}_{B^{+}}(z) \prod_{j=1}^{\tilde{m}} \tilde{l}_{j}(z)
$$

has a holomorphic continuation to $T_{\tilde{B}}$ and $g(\mathbf{0}) \neq 0$.

Proof. a) Define $c_{0}: M_{\mathbf{R}, \infty} \rightarrow \mathbf{R}_{\geq 0}$ by

$$
c_{0}\left(\left(m_{v}\right)_{v}\right)=\prod_{v \mid \infty}\left\{\sum_{\operatorname{dim} \sigma=d} \prod_{e \in \sigma \cap \Sigma_{1}} \frac{1}{\left(1+\left|e\left(m_{v}\right)\right|\right)^{1+1 / 2 d}}\right\} .
$$

Let $\mathcal{F} \subset M_{\mathbf{R}, \infty}^{1}$ be the cube spanned by a basis of the image of $\mathcal{U}_{T}$ in $M_{\mathbf{R}, \infty}^{1}$. Let $c^{\prime}>0$ such that for all $m_{\infty}(\chi) \in M_{\mathbf{R}, \infty}^{1}\left(\chi \in \mathcal{U}_{T}\right)$ and all $m^{1} \in \mathcal{F}$

$$
c_{0}\left(m_{\infty}(\chi)\right) \leq c^{\prime} c_{0}\left(m_{\infty}(\chi)+m^{1}\right) .
$$

Let $d m^{1}$ be the Lebesgue measure on $M_{\mathbf{R}, \infty}^{1}$ normalized by the image of $\mathcal{U}_{T}$. Then for $m \in M_{\mathbf{R}}$

$$
\int_{M_{\mathbf{R}}^{\prime}}\left\{\sum_{\chi \in \mathcal{U}_{T}} c_{0}\left(m_{\infty}(\chi)+m^{\prime}+m\right)\right\} d m^{\prime} \leq c\left(m \bmod M_{\mathbf{R}}^{\prime}\right),
$$

where $c: M_{\mathbf{R}}^{\prime \prime} \rightarrow \mathbf{R}_{\geq 0}$ is defined by

$$
c\left(m^{\prime \prime}\right):=\operatorname{cl}_{F}^{d} c^{\prime} \int_{M_{\mathbf{R}}^{\prime}} \int_{M_{\mathbf{R}, \infty}^{1}} c_{0}\left(m^{\prime}+m^{1}+m^{\prime \prime}\right) d m^{1} d m^{\prime} .
$$

By Lemma 5.4, for any compact subset $\mathbf{K}$ of $T_{B}$ there is a $\kappa(\mathbf{K})>0$ such that

$$
\left|g\left(z+i m^{\prime \prime}\right)\right| \leq \kappa(\mathbf{K}) c\left(m^{\prime \prime}\right)
$$


for all $z \in \mathbf{K}$ and $m^{\prime \prime} \in M_{\mathbf{R}}^{\prime \prime}$. Obviously, $c$ can be integrated over any subspace $U$ of $M_{\mathbf{R}}^{\prime \prime}$. It remains to show that for any $m^{\prime \prime} \in M_{\mathbf{R}}^{\prime \prime}-U$ one has

$$
\lim _{\tau \rightarrow \pm \infty} \int_{U} c\left(\tau m^{\prime \prime}+u\right) d u=0 .
$$

In section 7.6 it will be shown that $c$ is a sufficient function. Thus $f$ is a distinguished function in the sense of 5.2 .

b) The first part concerning the meromorphic continuation and singularities of $\tilde{f}_{B^{+}}$is the content of Theorem 5.2. The relation

$$
\lim _{s \rightarrow 0} s^{b(L)} \tilde{f}_{B^{+}}(s \hat{L})=g(\mathbf{0}) \mathcal{X}_{\Lambda}\left(\psi_{0}(\hat{L})\right)
$$

is satisfied by Theorem 5.3 and (19). It will be shown in Section 6 that $g(\mathbf{0}) \neq 0$.

5.6 The main theorem of our paper is:

Theorem 5.6 Let $L$ be a line bundle on $Y$ which lies in the interior of the cone of effective divisors. Then there exists a metrization $\mathcal{L}$ of $L$ with the following properties:

a) The height zeta function

$$
Z_{Y^{o}}(\mathcal{L}, s)=\sum_{y \in Y^{o}(F)} H_{\mathcal{L}}(y)^{-s}
$$

is holomorphic for $\operatorname{Re}(s)>a(L)$ and it can be continued meromorphically to a halfspace $\operatorname{Re}(s)>a(L)-\delta$ for some $\delta>0$. In this halfspace it has a pole of order $b(L)$ at $a(L)$ and no other poles.

b) For the counting function one has the following asymptotic relation

$$
N_{Y^{o}}(\mathcal{L}, H)=c(\mathcal{L}) H^{a(L)}(\log H)^{b(L)-1}(1+o(1))
$$

for $H \rightarrow \infty$ with some constant $c(\mathcal{L})>0$.

Proof. a) By construction, $\hat{L}=\frac{1}{a(L)}\left(\varphi_{\Sigma}+\varphi_{L}, 2 \lambda_{P}+\lambda_{L}\right)$ is mapped onto $[L]$ by $\psi$. Hence $Z_{Y^{o}}(\mathcal{L}, s)$ converges absolutely for $\operatorname{Re}(s)>a(L)$, where $\mathcal{L}$ is the metrization mentioned in Proposition 4.4. By the same proposition,

$$
Z_{Y^{o}}(\mathcal{L}, s+a(L))=\mu_{T} \int_{M_{\mathbf{R}}}\left\{\sum_{\chi \in \mathcal{U}_{T}} f_{L}(\chi, i m)\right\} d m
$$


where

$$
\begin{aligned}
& f_{L}(\chi, i m):= \\
& \hat{H}_{\Sigma}\left(\chi, \frac{s}{a(L)}\left(\varphi_{\Sigma}+\varphi_{L}\right)+\varphi_{\Sigma}+\varphi_{L}+i m\right) E_{P}^{G}\left(\frac{s}{a(L)}\left(2 \rho_{P}+\lambda_{L}\right)+\rho_{P}+\lambda_{L}-i \check{\eta}(m), \chi_{\eta}^{-1}\right)
\end{aligned}
$$

for all $s$ with $\operatorname{Re}(s)>0$. However, this is just

$$
\frac{1}{(2 \pi)^{d^{\prime \prime}}} \int_{M_{\mathbf{R}}^{\prime \prime}} f\left(s \hat{L}+i\left(m^{\prime \prime},-\check{\eta}\left(m^{\prime \prime}\right)\right)\right) d m^{\prime \prime}=\tilde{f}_{B^{+}}(s \hat{L})
$$

with $f, B^{+}$and $\tilde{f}_{B^{+}}$introduced in the preceding section. By Theorem $5.5, \tilde{f}_{B^{+}}$extends to a meromorphic function on a tube domain over a neighborhood of $\mathbf{0}$ and in this tube domain the only singularities are the hyperplanes defined over $\mathbf{R}$. Hence there is a $\delta>0$ such that $Z_{Y^{o}}(\mathcal{L}, s+a(L))$ extends to a meromorphic function in the half-space $\operatorname{Re}(s)>-\delta$ and the only possible pole is in $s=0$ and its order is exactly $b(L)$ (Theorems 5.3 and $5.5)$.

b) This result follows from a Tauberian theorem (cf. [11], Théorème III or [42], Problem 14.1 (in the constant stated there the factor $\frac{1}{k_{0}}$ is missing)).

\section{Non-vanishing of asymptotic constants}

6.1 This section is devoted to the proof of the non-vanishing of $g(\mathbf{0})$ claimed in Theorem 5.5. All notations are as in sections 5.2-5.5. The function $g(\varphi, \lambda)$ which has been defined in 5.5 is given by

$$
g(\varphi, \lambda)=\frac{\mu_{T^{\prime}}}{\kappa^{d^{\prime \prime}}} \int_{M^{\prime}}\left\{\sum_{\chi \in \mathcal{U}_{T}} \hat{H}_{\Sigma}\left(\chi, \tilde{\varphi}+i m^{\prime}\right) E_{P}^{G}\left(\tilde{\lambda}-i \check{\eta}\left(m^{\prime}\right), \chi_{\eta}^{-1}\right) h_{L}(\varphi, \lambda)\right\} d m
$$

where $\tilde{\varphi}, \tilde{\lambda}$ have been defined in 5.4. The function $h_{L}(\varphi, \lambda)$ was defined in 5.4:

$$
h_{L}(\varphi, \lambda)=\prod_{e \in \Sigma_{1}-\Sigma_{1}^{\prime}} \frac{\varphi(e)}{\varphi(e)+1} \prod_{\alpha \in \Delta_{P}-\Delta_{P^{\prime}}} \frac{\langle\lambda, \alpha\rangle}{\langle\lambda, \alpha\rangle+1} .
$$

The uniform convergency of the integral above in any compact subset of $T_{B}$ (cf. Lemma 5.4) allows us to compute the limit

$$
\lim _{(\varphi, \lambda) \rightarrow \mathbf{0}} \prod_{e \in \Sigma_{1}-\Sigma_{1}^{\prime}} \varphi(e) \prod_{\alpha \in \Delta_{P}-\Delta_{P^{\prime}}}\langle\lambda, \alpha\rangle \hat{H}_{\Sigma}\left(\chi, \tilde{\varphi}+i m^{\prime}\right) E_{P}^{G}\left(\tilde{\lambda}-i \check{\eta}\left(m^{\prime}\right), \chi_{\eta}^{-1}\right)
$$


first and then to integrate. We shall show that this limit vanishes if there are $e \in \Sigma_{1}-\Sigma_{1}^{\prime}$ with $\chi_{e} \neq 1$ or $\alpha \in \Delta_{P}-\Delta_{P^{\prime}}$ with $\chi_{\eta} \circ \check{\alpha} \neq 1$. Therefore, we may consider only $\chi \in \mathcal{U}_{T}^{\prime}$ where

$$
\mathcal{U}_{T}^{\prime}:=\left\{\chi \in \mathcal{U}_{T} \mid \chi_{e}=1 \forall e \in \Sigma_{1}-\Sigma_{1}^{\prime}, \chi_{\eta} \circ \check{\alpha}=1 \forall \alpha \in \Delta_{P}-\Delta_{P^{\prime}}\right\} .
$$

Let $\eta^{\prime}: P^{\prime} \rightarrow T$ be the uniquely defined homomorphism such that for all $\alpha \in \Delta_{P^{\prime}}$ we have $\eta^{\prime} \circ \check{\alpha}=\eta \circ \check{\alpha}$.

\section{Lemma 6.1}

$$
\begin{aligned}
g(\mathbf{0})=\lim _{\varphi \rightarrow \mathbf{0}, \varphi \in P L(\Sigma)^{+}} \prod_{e \in \Sigma_{1}-\Sigma_{1}^{\prime}} \varphi(e) \cdot \frac{\mu_{T^{\prime}}}{\kappa^{d^{\prime \prime}}} & \cdot \frac{c_{P^{\prime}}}{c_{P}} \int_{M_{\mathbf{R}}^{\prime}}\left\{\sum_{\chi \in \mathcal{U}_{T}^{\prime}} \hat{H}_{\Sigma}\left(\chi, \tilde{\varphi}+i m^{\prime}\right)\right. \\
& \left.\times E_{P^{\prime}}^{G}\left(\lambda_{L}+\rho_{P^{\prime}}-i \check{\eta}^{\prime}\left(m^{\prime}\right),\left(\chi_{\eta^{\prime}}\right)^{-1}\right)\right\} d m^{\prime}
\end{aligned}
$$

(cf. 8.4 for the definition of $c_{P^{\prime}}$ and $c_{P}$ ).

Proof. Recall that (cf. (12))

$$
\hat{H}_{\Sigma}\left(\chi, \tilde{\varphi}+i m^{\prime}\right)=\zeta_{\Sigma}\left(\chi, \tilde{\varphi}+i m^{\prime}\right) \prod_{e \in \Sigma_{1}} L_{f}\left(\chi_{e}, 1+\varphi(e)+\varphi_{L}(e)+i e\left(m^{\prime}\right)\right)
$$

and that $\zeta_{\Sigma}\left(\chi, \tilde{\varphi}+i m^{\prime}\right)$ is regular for $\varphi$ in a tube domain over a neighborhood of $\mathbf{0}$ (Lemma 3.5). For $e \in \Sigma_{1}^{\prime}$ we have $\varphi_{L}(e)>0$; hence we see that the function

$$
L_{f}\left(\chi_{e}, 1+\varphi(e)+\varphi_{L}(e)+i e\left(m^{\prime}\right)\right)
$$

is holomorphic for $\operatorname{Re}(\varphi)$ in a neighborhood of $\mathbf{0}$. Let $e \in \Sigma_{1}-\Sigma_{1}^{\prime}$. If $\chi_{e} \neq 1$, then the restriction of $\chi_{e}$ to $\mathbf{G}_{m}(\mathbf{A})^{1}$ is non-trivial (by our construction of the embedding $\mathcal{U}_{T} \rightarrow \mathcal{A}_{T}$, cf. 3.3); hence

$$
\varphi \mapsto L_{f}\left(\chi_{e}, 1+\varphi(e)\right)
$$

is an entire function and $\varphi(e) L_{f}\left(\chi_{e}, 1+\varphi(e)\right)$ tends to 0 as $\varphi \rightarrow \mathbf{0}$.

For $\alpha \in \Delta_{P^{\prime}}$ we have $\left\langle\lambda_{L}, \alpha\right\rangle>0$; hence $\lambda_{L}$ is contained in $X^{*}\left(P^{\prime}\right)^{+}$. Let $\alpha \in$ $\Delta_{P}-\Delta_{P^{\prime}}$. If $\chi_{\eta} \circ \check{\alpha} \neq 1$, then $\chi_{\eta} \circ \check{\alpha}$ restricted to $\mathbf{G}_{m}(\mathbf{A})^{1}$ is non-trivial and therefore

$$
\prod_{\alpha \in \Delta_{P}-\Delta_{P^{\prime}}}\langle\lambda, \alpha\rangle E_{P}^{G}\left(\lambda+\rho_{P}+\lambda_{L}-i \check{\eta}\left(m^{\prime}\right), \chi_{\eta}^{-1}\right)
$$

vanishes as $\lambda \rightarrow \mathbf{0}$ (cf. Proposition 8.3). We have shown that it suffices to take the sum over all $\chi \in \mathcal{U}_{T}^{\prime}$. To complete the proof, note that for $\chi \in \mathcal{U}_{T}^{\prime}$ we have (cf. Proposition $(8.4))$

$$
\lim _{\lambda \rightarrow \mathbf{0}} \prod_{\alpha \in \Delta_{P}-\Delta_{P^{\prime}}}\langle\lambda, \alpha\rangle E_{P}^{G}\left(\tilde{\lambda}-i \check{\eta}\left(m^{\prime}\right), \chi_{\eta}^{-1}\right)=\frac{c_{P^{\prime}}}{c_{P}} E_{P^{\prime}}^{G}\left(\lambda_{L}+\rho_{P^{\prime}}-i \check{\eta}^{\prime}\left(m^{\prime}\right),\left(\chi_{\eta}^{\prime}\right)^{-1}\right) .
$$


6.2 By the absolute and uniform convergence of

$$
\int_{M_{\mathbf{R}}^{\prime}}\left\{\sum_{\chi \in \mathcal{U}_{T}^{\prime}} \hat{H}_{\Sigma}\left(\chi, \tilde{\varphi}+i m^{\prime}\right) \prod_{e \in \Sigma_{1}-\Sigma_{1}^{\prime}} \varphi(e)\right\} d m^{\prime}
$$

(cf. Lemma 3.5 and Theorem 3.6) and the convergence of

$$
\sum_{\gamma \in P^{\prime}(F) \backslash G(F)} e^{\left\langle\lambda_{L}+2 \rho_{P^{\prime}}, H_{P^{\prime}}(\gamma)\right\rangle}
$$

we may change summation and integration in (20) and get for all $\varphi \in P L(\Sigma)^{+}$

$$
\begin{aligned}
& \frac{\mu_{T^{\prime}}}{\kappa^{d^{\prime \prime}}} \cdot \frac{c_{P^{\prime}}}{c_{P}} \int_{M_{\mathbf{R}}^{\prime}} \sum_{\chi \in \mathcal{U}_{T}^{\prime}} \hat{H}_{\Sigma}\left(\chi, \tilde{\varphi}+i m^{\prime}\right) E_{P^{\prime}}^{G}\left(\lambda_{L}+\rho_{P^{\prime}}-i \check{\eta}^{\prime}\left(m^{\prime}\right)\left(\chi_{\eta^{\prime}}\right)^{-1}\right) d m^{\prime} \\
& =\frac{c_{P^{\prime}}}{c_{P} \kappa^{d^{\prime \prime}}} \sum_{\gamma \in P^{\prime}(F) \backslash G(F)} e^{\left\langle\lambda_{L}+2 \rho_{P^{\prime}}, H_{P^{\prime}}\left(\gamma^{\prime}\right)\right\rangle} \mu_{T^{\prime}} \int_{M_{\mathbf{R}}^{\prime}} \sum_{\chi \in \mathcal{U}_{T}^{\prime}} \hat{H}_{\Sigma}\left(\chi, \tilde{\varphi}+i m^{\prime}\right)\left(\chi^{m^{\prime}} \chi\right)^{-1}\left(\eta^{\prime}\left(p_{\gamma}^{\prime}\right)\right) d m^{\prime}
\end{aligned}
$$

where $\gamma=p_{\gamma}^{\prime} k_{\gamma}$ as above. Let $I$ be the image of the homomorphism

$$
\prod_{e \in \Sigma_{1}-\Sigma_{1}^{\prime}} \mathbf{G}_{m}(\mathbf{A}) \times \prod_{\alpha \in \Delta_{P}-\Delta_{P^{\prime}}} \mathbf{G}_{m}(\mathbf{A}) \rightarrow T(\mathbf{A})
$$

induced by $M \rightarrow \mathbf{Z}^{\Sigma_{1}-\Sigma_{1}^{\prime}} \oplus \mathbf{Z}^{\Delta_{P}-\Delta_{P^{\prime}}}$,

$$
\left.m \mapsto\left((e(m))_{e \in \Sigma_{1}-\Sigma_{1}^{\prime}},(\langle-m \circ \eta, \alpha\rangle)_{\alpha \in \Delta_{P}-\Delta_{P^{\prime}}}\right)\right) .
$$

Then $M_{\mathbf{R}}^{\prime} \oplus \mathcal{U}_{T}^{\prime}$ is precisely the set of characters $T(\mathbf{A}) \rightarrow S^{1}$ which are trivial on $T(F) \mathbf{K}_{T} I$. Put $T^{\prime}=\operatorname{Spec}\left(F\left[M^{\prime}\right]\right)$ and $T^{\prime \prime}=\operatorname{Spec}\left(F\left[M^{\prime \prime}\right]\right)$. Then there is an exact sequence

$$
1 \rightarrow T^{\prime \prime} \rightarrow T \rightarrow T^{\prime} \rightarrow 1
$$

Note that $I \subset T^{\prime \prime}(\mathbf{A})$. Denote by $\mathbf{K}_{T^{\prime}}$ (resp. $\left.\mathbf{K}_{T^{\prime \prime}}\right)$ the maximal compact subgroup of $T^{\prime}(\mathbf{A})$ (resp. of $T^{\prime \prime}(\mathbf{A})$ ). The linear forms

$$
\begin{gathered}
m \mapsto e(m), \quad e \in \Sigma_{1}-\Sigma_{1}^{\prime}, \\
m \mapsto-\langle m \circ \eta, \alpha\rangle, \quad \alpha \in \Delta_{P}-\Delta_{P^{\prime}}
\end{gathered}
$$


when considered as functions on $M^{\prime \prime}$, generate a sublattice of finite index in $N^{\prime \prime}=$ $\operatorname{Hom}\left(M^{\prime \prime}, \mathbf{Z}\right)$. This shows that there is a $q>0$ such that the image of the $q$-th power homomorphism $T^{\prime \prime}(\mathbf{A}) \rightarrow T^{\prime \prime}(\mathbf{A}), t \mapsto t^{q}$, is contained in $I$. If $v$ is any archimedean place of $F$ the connected component of one in $T^{\prime \prime}\left(F_{v}\right)$ is therefore contained in $I$. Consequently,

$$
T^{\prime \prime}(F) \cdot \prod_{v \mid \infty} T^{\prime \prime}\left(F_{v}\right) \prod_{v \nmid \infty} \mathbf{K}_{T^{\prime \prime}, v} \subset T^{\prime \prime}(F) \mathbf{K}_{T^{\prime \prime}} \cdot I
$$

and the left hand side is of finite index in $T^{\prime \prime}(\mathbf{A})$. Put

$$
\mathcal{A}_{T}^{\prime}=\left\{\chi \in \mathcal{A}_{T} \mid \chi=1 \text { on } T(F) \mathbf{K}_{T} T^{\prime \prime}(\mathbf{A})\right\} .
$$

We observe that

$$
\mathcal{A}_{T}^{\prime} \simeq \mathcal{A}_{T^{\prime}}=\left(T^{\prime}(\mathbf{A}) / T^{\prime}(F) \mathbf{K}_{T^{\prime}}\right)^{*} \subset M_{\mathbf{R}}^{\prime} \oplus \mathcal{U}_{T}^{\prime}
$$

We denote by

$$
\mathcal{I}=\left(M_{\mathbf{R}}^{\prime} \oplus \mathcal{U}_{T}^{\prime}\right) / \mathcal{A}_{T}^{\prime}
$$

and by $\iota$ the order of $\mathcal{I}$. Put $\mathcal{U}_{T^{\prime}}=\mathcal{U}_{T} \cap \mathcal{A}_{T}^{\prime}$ (then $\mathcal{A}_{T}^{\prime}=M_{\mathbf{R}}^{\prime} \oplus \mathcal{U}_{T^{\prime}}$ ). Thus we may write

$$
\begin{aligned}
\int_{M_{\mathbf{R}}^{\prime}}\left\{\sum_{\chi \in \mathcal{U}_{T}^{\prime}} \hat{H}_{\Sigma}\left(\chi, \tilde{\varphi}+i m^{\prime}\right)\right. & \left.\left(\chi^{m^{\prime}} \chi\right)^{-1}\left(\eta\left(p_{\gamma}^{\prime}\right)\right)\right\} d m^{\prime} \\
& =\sum_{\chi \in \mathcal{I}} \int_{M_{\mathbf{R}}^{\prime}}\left\{\sum_{\chi^{\prime} \in \mathcal{U}_{T^{\prime}}} \hat{H}_{\Sigma}\left(\chi^{\prime} \chi^{m^{\prime}} \chi, \tilde{\varphi}\right)\left(\chi^{\prime} \chi^{m^{\prime}} \chi\right)^{-1}\left(\eta^{\prime}\left(p_{\gamma}^{\prime}\right)\right)\right\} d m^{\prime} .
\end{aligned}
$$

For $\chi \in M_{\mathbf{R}}^{\prime} \oplus \mathcal{U}_{T}^{\prime}$ and $x \in T^{\prime}(\mathbf{A})$ we consider the function

$$
x \mapsto \int_{T^{\prime \prime}(\mathbf{A})} H_{\Sigma}\left(x t \eta^{\prime}\left(p_{\gamma}^{\prime}\right), \tilde{\varphi}\right) \chi(t) d t
$$

(the Haar measure $d t$ on $T^{\prime \prime}(\mathbf{A})$ is defined as $d x$ on $T(\mathbf{A})$, cf. 3.3). The same argument as in the proof of Proposition 3.4 shows that this function is absolutely integrable over $T^{\prime}(F)$ if $\varphi \in P L(\Sigma)^{+}$. The Fourier transform for $\chi^{\prime} \in \mathcal{A}_{T}^{\prime}$

$$
\int_{T^{\prime}(\mathbf{A})}\left(\int_{T^{\prime \prime}(\mathbf{A})} H_{\Sigma}\left(x t \eta^{\prime}\left(p_{\gamma}^{\prime}\right), \tilde{\varphi}\right) \chi(t) d t\right) \chi^{\prime}(x) d x
$$


is absolutely integrable over $\mathcal{A}_{T}^{\prime}$. Using Poisson's summation formula twice we get

$$
\begin{aligned}
\sum_{\chi \in \mathcal{I}} \mu_{T^{\prime}} & \int_{M_{\mathbf{R}}^{\prime}}\left\{\sum_{\chi^{\prime} \in \mathcal{U}_{T^{\prime}}} \hat{H}_{\Sigma}\left(\chi^{m^{\prime}} \chi^{\prime} \chi, \tilde{\varphi}\right)\left(\chi^{\prime} \chi^{m^{\prime}} \chi\right)^{-1}\left(\eta^{\prime}\left(p_{\gamma}^{\prime}\right)\right)\right\} d m^{\prime} \\
= & \sum_{\chi \in \mathcal{I}} \sum_{x \in T^{\prime}(F)} \int_{T^{\prime \prime}(\mathbf{A})} H_{\Sigma}\left(x t \eta^{\prime}\left(p_{\gamma}^{\prime}, \tilde{\varphi}\right) \chi(t) d t=\sum_{x \in T^{\prime}(F)} \iota \int_{T^{\prime \prime}(F) \mathbf{K}_{T} I} H_{\Sigma}\left(x t \eta^{\prime}\left(p_{\gamma}^{\prime}\right), \tilde{\varphi}\right) d t .\right.
\end{aligned}
$$

Now we collect all the terms together.

Lemma 6.2 The constant $g(\mathbf{0})$ is equal to

$$
\begin{aligned}
& \frac{\iota c_{P^{\prime}} / c_{P}}{\kappa^{d^{\prime \prime}}} \sum_{\gamma \in P^{\prime}(F) \backslash G(F)} e^{\left\langle\lambda_{L}+2 \rho_{P^{\prime}}, H_{P^{\prime}}(\gamma)\right\rangle} \times \\
& \lim _{\varphi \rightarrow \mathbf{0}, \varphi \in P L(\Sigma)^{+}} \prod_{e \in \Sigma_{1}-\Sigma_{1}^{\prime}} \varphi(e) \sum_{x \in T^{\prime}(F)} \int_{T^{\prime \prime}(F) \mathbf{K}_{T^{\prime \prime}} I} H_{\Sigma}\left(x \eta^{\prime}\left(p_{\gamma}^{\prime}\right) t, \tilde{\varphi}\right) d t .
\end{aligned}
$$

Lemma 6.3 The limit

$$
\lim _{\varphi \rightarrow \mathbf{0}, \varphi \in P L(\Sigma)^{+}} \prod_{e \in \Sigma_{1}-\Sigma_{1}^{\prime}} \varphi(e) \int_{T^{\prime \prime}(\mathbf{A})} H_{\Sigma}(t, \tilde{\varphi}) d t
$$

exists and is positive.

Proof. Consider the embedding $N_{\mathbf{R}}^{\prime \prime} \rightarrow N_{\mathbf{R}}$ and let

$$
\Sigma^{\prime \prime}:=\left\{\sigma \cap N_{\mathbf{R}}^{\prime \prime} \mid \sigma \in \Sigma\right\} .
$$

This is a complete fan in $N_{\mathbf{R}}^{\prime \prime}$ which consists of rational polyhedral cones, but which is not necessary a regular fan. We can obtain a regular fan by subdivision of the cones into regular ones (cf. [20], ch. I, $\S 2$, Theorem 11). This gives us a complete regular fan $\tilde{\Sigma}^{\prime \prime}$ such that any cone in $\tilde{\Sigma}^{\prime \prime}$ is contained in a cone of $\Sigma^{\prime \prime}$. Denote by $\tilde{\Sigma}_{1}^{\prime \prime}$ the set of primitive integral generators of the one-dimensional cones in $\tilde{\Sigma}^{\prime \prime}$. Computing the integral as in section 3.4 we get

$$
\int_{T^{\prime \prime}(\mathbf{A})} H_{\Sigma}(t, \tilde{\varphi}) d t=\zeta_{\tilde{\Sigma}^{\prime \prime}}(1, \tilde{\varphi}) \prod_{\tilde{e} \in \tilde{\Sigma}_{1}^{\prime \prime}} L_{f}(1, \tilde{\varphi}(\tilde{e}))
$$


(cf. (12)), where $\zeta_{\tilde{\Sigma}^{\prime \prime}}(1, \tilde{\varphi})$ is regular in a neighborhood of $\varphi=\mathbf{0}$ and positive for $\varphi=\mathbf{0}$. Let $\tilde{e} \in N$ and $\sigma \in \Sigma^{\prime}$ be a cone containing $\tilde{e}$. Write $\tilde{e}=\sum_{e \in \sigma \cap \Sigma_{1}} t_{e} \cdot e\left(t_{e} \in \mathbf{Z}_{\geq 0}\right)$. Suppose

$$
1=\left(\varphi_{\Sigma}+\varphi_{L}\right)(\tilde{e})=\sum_{e \in \sigma \cap \Sigma_{1}} t_{e}\left(1+\varphi_{L}(e)\right) .
$$

Then $t_{e}=0$ for all $e \in \sigma \cap \Sigma_{1}^{\prime}$ because $\varphi_{L} \in \sum_{e \in \Sigma_{1}^{\prime}} \mathbf{R}_{>0} \varphi_{e}$ (cf. 5.4). Hence,

$$
\tilde{e}=\sum_{e \in \sigma \cap \Sigma_{1}-\Sigma_{1}^{\prime}} t_{e} \cdot e
$$

and $\varphi_{\Sigma}(\tilde{e})=1$ implies $\tilde{e} \in \Sigma_{1}-\Sigma_{1}^{\prime}$. Therefore,

$$
\begin{aligned}
& \lim _{\varphi \rightarrow \mathbf{0}, \varphi \in P L(\Sigma)^{+}} \prod_{e \in \Sigma_{1}-\Sigma_{1}^{\prime}} \varphi(e) \prod_{\tilde{e} \in \tilde{\Sigma}_{1}^{\prime \prime}} L_{f}(1, \tilde{\varphi}(\tilde{e})) \\
& \quad=\left\{\prod_{e \in \Sigma_{1}-\Sigma_{1}^{\prime}} \lim _{\varphi \rightarrow \mathbf{0}, \varphi \in P L(\Sigma)^{+}} \varphi(e) L_{f}(1,1+\varphi(e))\right\} \prod_{\tilde{e} \in \tilde{\Sigma}_{1}^{\prime \prime}-\left(\Sigma_{1}-\Sigma_{1}^{\prime}\right)} L_{f}\left(1,\left(\varphi_{\Sigma}+\varphi_{L}\right)(\tilde{e})\right)
\end{aligned}
$$

and this is a positive real number.

In Theorem 5.5 we claimed the non-vanishing of $g(\mathbf{0})$. We are now in the position to prove

Corollary 6.4 The function $g$ defined in section 5.5 does not vanish in $(\varphi, \lambda)=(\mathbf{0}, \mathbf{0})$ :

$$
g(\mathbf{0})>0
$$

Proof. By Lemma 6.2 it is enough to show that

$$
\lim _{\varphi \rightarrow \mathbf{0}, \varphi \in P L(\Sigma)^{+}} \prod_{e \in \Sigma_{1}-\Sigma_{1}^{\prime}} \varphi(e) \int_{T^{\prime \prime}(F) \mathbf{K}_{T^{\prime \prime}} \cdot I} H_{\Sigma}(t, \tilde{\varphi}) d t
$$

is positive. Let $t_{1}, \ldots, t_{\nu} \in T(\mathbf{A})$ be such that

$$
T^{\prime \prime}(\mathbf{A})=\bigcup_{j=1}^{\nu} t_{j} T^{\prime \prime}(F) \mathbf{K}_{T^{\prime \prime}} \cdot I
$$


Then there exists a constant $c>0$ such that for all $t \in T^{\prime \prime}(\mathbf{A})$ and $j=1, \ldots, \nu$, we have

$$
H_{\Sigma}\left(t t_{j}, \tilde{\varphi}\right) \leq \frac{c}{\nu} H_{\Sigma}(t, \tilde{\varphi})
$$

Hence we can estimate

$$
\int_{T^{\prime \prime}(\mathbf{A})} H_{\Sigma}(t, \tilde{\varphi}) d t=\sum_{j=1}^{\nu} \int_{T^{\prime \prime}(F) \mathbf{K}_{T^{\prime \prime}} I} H_{\Sigma}\left(t t_{j}, \tilde{\varphi}\right) d t \leq c \int_{T^{\prime \prime}(F) \mathbf{K}_{T^{\prime \prime}} I} H_{\Sigma}(t, \tilde{\varphi}) d t .
$$

Lemma 6.3 allows us to conclude that the limit above is indeed positive.

\section{Technical theorems}

7.1 Let $(A, V, \Lambda)$ be a triple consisting of a free abelian group $A$ of rank $d$, a $d$ dimensional real vector space $V:=A \otimes \mathbf{R}$ containing $A$ as a sublattice of maximal rank, and a closed strongly convex polyhedral $d$-dimensional cone $\Lambda \subset A_{\mathbf{R}}$ such that $\Lambda \cap-\Lambda=\{\mathbf{0}\}$. Denote by $\Lambda^{\circ}$ the interior of $\Lambda$. Let $\left(A^{\vee}, V^{\vee}, \Lambda^{\vee}\right)$ be the triple consisting of the dual abelian group $A^{\vee}=\operatorname{Hom}(A, \mathbf{Z})$, the dual real vector space $V^{\vee}=\operatorname{Hom}(V, \mathbf{R})$ and the dual cone $\Lambda^{\vee} \subset V^{\vee}$. We normalize the Haar measure $d y$ on $V^{\vee}$ by the condition $\operatorname{Vol}\left(V^{\vee} / A^{\vee}\right)=1$.

We denote by $\chi_{\Lambda}(v)$ the set-theoretic characteristic function of the cone $\Lambda$ and by $\mathcal{X}_{\Lambda}(v)$ the Laplace transform of the set-theoretic characteristic function of the dual cone

$$
\mathcal{X}_{\Lambda}(v)=\int_{V^{\vee}} \chi_{\Lambda^{\vee}}(y) e^{-\langle v, y\rangle} d y=\int_{\Lambda^{\vee}} e^{-\langle v, y\rangle} d y,
$$

where $\operatorname{Re}(v) \in \Lambda^{\circ}$ (for these $v$ the integral converges absolutely).

Consider a complete regular fan $\Sigma$ on $V$, that is, a subdivision of the real space $V$ into a finite set of convex rational simplicial cones, satisfying certain conditions (see [3], 1.2). Denote by $\Sigma_{1}$ the set of primitive generators of one dimensional cones in $\Sigma$. Denote by $P L(\Sigma)_{\mathbf{R}}$ the vector space of real valued piecewise linear functions on $V$ and by $P L(\Sigma)_{\mathbf{C}}$ its complexification.

Proposition 7.1 ([3], Prop. 2.3.2, p. 614) For any compact $\mathbf{K} \subset P L(\Sigma)_{\mathbf{C}}$ with the property that $\operatorname{Re}(\varphi(v))>0$ for all $\varphi \in \mathbf{K}$ and $v \neq \mathbf{0}$, there exists a constant $\kappa(\mathbf{K})$ such that for all $\varphi \in \mathbf{K}$ and all $y \in V^{\vee}$ the following inequality holds

$$
\left|\int_{V} e^{-\varphi(v)-i\langle v, y\rangle} d v\right| \leq \kappa(\mathbf{K}) \sum_{\operatorname{dim} \sigma=d} \frac{1}{\prod_{e \in \sigma}(1+|\langle e, y\rangle|)^{1+1 / d}} .
$$


7.2 Let $H \subset V$ be a hyperplane with $H \cap \Lambda=\{\mathbf{0}\}$. Let $y_{0} \in V^{\vee}$ with $H=\operatorname{Ker}\left(y_{0}\right)$, such that for all $x \in \Lambda: y_{0}(x) \geq 0$. Then $y_{0}$ is in the interior of $\Lambda^{\vee} \subset V^{\vee}$. Let $x_{0} \in \Lambda^{\circ}$ and let

$$
H^{\prime}=\left\{y^{\prime} \in V^{\vee} \mid y^{\prime}\left(x_{0}\right)=0\right\} .
$$

We have $V^{\vee}=H^{\prime} \oplus \mathbf{R} y_{0}$. Define $\varphi: H^{\prime} \rightarrow \mathbf{R}$ by

$$
\varphi\left(y^{\prime}\right)=\min \left\{t \mid y^{\prime}+t y_{0} \in \Lambda^{\vee}\right\}
$$

The function $\varphi$ is piecewise linear with respect to a complete fan of $H^{\prime}$. Taking a subdivision, if necessary, we may assume it to be regular.

Proposition 7.2 The function $\mathcal{X}_{\Lambda}(u)$ is absolutely integrable over any linear subspace $U \subset H$.

Proof. For $h \in H$ we have

$$
\begin{aligned}
\mathcal{X}_{\Lambda}\left(x_{0}+i h\right)=\int_{\Lambda^{\vee}} e^{-y\left(x_{0}+i h\right)} d y=\int_{H^{\prime}} \int_{\varphi\left(y^{\prime}\right)}^{\infty} e^{-\left(y^{\prime}+t y_{0}\right)\left(x_{0}+i h\right)} d t d y^{\prime} & \\
& =\int_{H^{\prime}} e^{-\varphi\left(y^{\prime}\right)} e^{-i y^{\prime}(h)} d y^{\prime}
\end{aligned}
$$

Therefore, $h \mapsto \mathcal{X}_{\Lambda}\left(x_{0}+i h\right)$ is the Fourier transform of the function $y^{\prime} \mapsto e^{-\varphi\left(y^{\prime}\right)}$ on $H^{\prime} \simeq H^{\vee}$. The statement follows now from 7.1 .

7.3 The rest of this section is devoted to the proof of the meromorphic continuation of certain functions which are holomorphic in tube domains over convex finitely generated polyhedral cones. In section 5 we have already introduced the terminology and explained how this technical theorem is applied to height zeta functions.

Let $E$ be a finite dimensional vector space over $\mathbf{R}$ and $E_{\mathbf{C}}$ its complexification. Let $V \subset E$ be a subspace. We will call a function $c: V \rightarrow \mathbf{R}_{\geq 0}$ sufficient if it satisfies the following conditions:

(i) For any subspace $U \subset V$ and any $v \in V$, the function $U \rightarrow \mathbf{R}$ defined by $u \rightarrow$ $c(v+u)$ is measurable on $U$ and the integral

$$
c_{U}(v):=\int_{U} c(v+u) d u
$$

is always finite $(d u$ is a Lebesgue measure on $U)$. 
(ii) For any subspace $U \subset V$ and every $v \in V-U$ we have

$$
\lim _{\tau \rightarrow \pm \infty} c_{U}(\tau \cdot v)=0
$$

Let $l_{1}, \ldots, l_{m} \in E^{\vee}=\operatorname{Hom}_{\mathbf{R}}(E, \mathbf{R})$ be linearly independent linear forms. Put $H_{j}=$ $\operatorname{Ker}\left(l_{j}\right)$ for $j=1, \ldots, m$. Let $B \subset E$ be an open and convex neighborhood of $\mathbf{0}$, such that for all $x \in B$ and all $j=1, \ldots, m$ we have $l_{j}(x)>-1$. We denote by $T_{B}:=B+i E \subset E_{\mathbf{C}}$ the complex tube domain over $B$. We denote by $\mathcal{M}\left(T_{B}\right)$ the set of meromorphic functions on $T_{B}$.

A meromorphic function $f \in \mathcal{M}\left(T_{B}\right)$ will be called distinguished with respect to the data $\left(V ; l_{1}, \ldots, l_{m}\right)$ if it satisfies the following conditions:

(i) The function

$$
g(z):=f(z) \prod_{j=1}^{m} \frac{l_{j}(z)}{l_{j}(z)+1}
$$

is holomorphic in $T_{B}$.

(ii) There exists a sufficient function $c: V \rightarrow \mathbf{R}_{\geq 0}$ such that for any compact $\mathbf{K} \subset T_{B}$ there is a constant $\kappa(\mathbf{K}) \geq 0$ such that for all $z \in \mathbf{K}$ and all $v \in V$, we have

$$
|g(z+i v)| \leq \kappa(\mathbf{K}) c(v)
$$

Let $C$ be a connected component of $B-\bigcup_{j=1}^{m} H_{j}$ and $T_{C}$ a tube domain over $C$. We will consider the following integral:

$$
\tilde{f}_{C}(z):=\frac{1}{(2 \pi)^{d}} \int_{V} f(z+i v) d v
$$

Here we denoted by $d=\operatorname{dim} V$ and by $d v$ a fixed Lebesgue measure on $V$.

Proposition 7.3 Assume that $f$ is an distinguished function with respect to $\left(V ; l_{1}, \ldots, l_{m}\right)$. Then the following holds:

a) $\tilde{f}_{C}: T_{C} \rightarrow \mathbf{C}$ is a holomorphic function.

b) There exist an open and convex neighborhood $\tilde{B}$ of $\mathbf{0}$, containing $C$, and linear forms $\tilde{l}_{1}, \ldots, \tilde{l}_{\tilde{m}}$, which vanish on $V$, such that

$$
z \rightarrow \tilde{f}_{C}(z) \prod_{j=1}^{\tilde{m}} \tilde{l}_{j}(z)
$$

has a holomorphic continuation to $T_{\tilde{B}}$. 
Proof. a) Let $\mathbf{K} \subset T_{C}$ be a compact subset and let $\kappa(\mathbf{K}) \geq 0$ be a real number such that for all $z \in \mathbf{K}$ and all $v \in V$, we have $|g(z+i v)| \leq \kappa(\mathbf{K}) c(v)$. Since $\mathbf{K}$ is a compact and $C$ doesn't intersect any of the hyperplanes $H_{j}$, there exist real numbers $c_{j} \geq 0$ for any $j=1, \ldots, m$, such that for all $z \in \mathbf{K}$ and $v \in V$, the following inequalities hold:

$$
\left|\frac{l_{j}(z+i v)+1}{l_{j}(z+i v)}\right| \leq c_{j} .
$$

Therefore, for $z \in \mathbf{K}$ and $v \in V$ we have

$$
|f(z+i v)| \leq c_{1} \cdots c_{m} \kappa(\mathbf{K}) c(v) .
$$

It follows that on every compact $\mathbf{K} \subset T_{C}$ the integral converges absolutely and uniformly to a holomorphic function $\tilde{f}_{C}$.

b) The proof proceeds by induction on $d=\operatorname{dim} V$. For $d=0$ there is nothing to prove. Assume that $d \geq 1$ and let $v_{0} \in V-\{\mathbf{0}\}$ be a vector such that both $v_{0},-v_{0} \in B$. We define $B_{1} \subset B$ as the set of all vectors $x \in B$ which satisfy the following two conditions: the vector $x \pm v_{0} \in B$ and $\left|\frac{l_{j}(x)}{l_{j}\left(v_{0}\right)}\right| \leq \frac{1}{2}$ for all $j \in\{1, \ldots, m\}$ with $l_{j}\left(v_{0}\right) \neq 0$. The set $B_{1}$ is a convex open neighborhood of $\mathbf{0} \in E$. Fix a vector $x_{0} \in C$. Without loss of generality we can assume that

$$
\left\{1, \ldots, m_{0}\right\}:=\left\{j \in\{1, \ldots, m\} \mid l_{j}\left(v_{0}\right) l_{j}\left(x_{0}\right)<0\right\}
$$

with $0 \leq m_{0} \leq m$. For $j \in\left\{1, \ldots, m_{0}\right\}, k \in\{1, \ldots, \widehat{j}, \ldots, m\}$ we define

$$
l_{j, k}(x):=l_{k}(x)-l_{j}(x) \frac{l_{k}\left(v_{0}\right)}{l_{j}\left(v_{0}\right)}, \quad H_{j, k}:=\operatorname{Ker}\left(l_{j, k}\right) \subset E .
$$

For all $j \in\left\{1, \ldots, m_{0}\right\}$ we have that $\left(l_{j, k}\right)_{1 \leq k \leq m, k \neq j}$ is a set of linearly independent linear forms on $E$. Moreover, for all $x \in B_{1}$ and $j \in\left\{1, \ldots, m_{0}\right\}$ we have

$$
x-\frac{l_{j}(x)}{l_{j}\left(v_{0}\right)} v_{0}=\left(1-\frac{l_{j}(x)}{l_{j}\left(v_{0}\right)}\right) x+\frac{l_{j}(x)}{l_{j}\left(v_{0}\right)}\left(x-v_{0}\right) \in B,
$$

in the case that $\frac{l_{j}(x)}{l_{j}\left(v_{0}\right)} \geq 0$ and, similarly, in the case that $\frac{l_{j}(x)}{l_{j}\left(v_{0}\right)}<0$

$$
x-\frac{l_{j}(x)}{l_{j}\left(v_{0}\right)} v_{0}=\left(1+\frac{l_{j}(x)}{l_{j}\left(v_{0}\right)}\right) x+\left(-\frac{l_{j}(x)}{l_{j}\left(v_{0}\right)}\right)\left(x+v_{0}\right) \in B .
$$


Therefore, for all $x \in B_{1}$ and $j \in\left\{1, \ldots, m_{0}\right\}, k \in\{1, \ldots, \hat{j}, \ldots, m\}$ we have

$$
l_{j, k}(x)=l_{k}\left(x-\frac{l_{j}(x)}{l_{j}\left(v_{0}\right)} v_{0}\right)>-1
$$

Let $C_{1}$ be a connected component of

$$
B_{1}-\left(\bigcup_{j=1}^{m_{0}} \bigcup_{1 \leq k \leq m, k \neq j} H_{j, k} \cup \bigcup_{j=1}^{m} H_{j}\right)
$$

which is contained in $C$. For $z \in T_{C}$ we define

$$
h_{C}(z):=\frac{1}{2 \pi} \int_{-\infty}^{+\infty} f\left(z+i \tau v_{0}\right) d \tau=\frac{1}{2 \pi i} \int_{\operatorname{Re}(\lambda)=0} f\left(z+\lambda v_{0}\right) d \lambda .
$$

As in (i) one shows that $h_{C}$ is a holomorphic function on $T_{C}$. For $x \in B_{1}$ and $\lambda \in[0,1]$ we have

$$
x+\lambda v_{0}=(1-\lambda) x+\lambda\left(x+v_{0}\right) \in B .
$$

If for some $z=x+i y \in T_{C_{1}}\left(x \in C_{1}\right)$ and $\lambda \in[0,1]+i \mathbf{R}, j \in\{1, \ldots, m\}$ we have

$$
l_{j}\left(z+\lambda v_{0}\right)=0
$$

then it follows that $l_{j}(x)+\operatorname{Re}(\lambda) l_{j}\left(v_{0}\right)=0$, and therefore, $l_{j}(x) l_{j}\left(v_{0}\right)<0$ (since $l_{j}(x)$ has the same sign as $\left.l_{j}\left(x_{0}\right)\right)$. Consequently, $j \in\left\{1, \ldots, m_{0}\right\}$.

For $z \in T_{C_{1}}$ and $j \in\left\{1, \ldots, m_{0}\right\}$ we put

$$
\lambda_{j}(z):=-\frac{l_{j}(z)}{l_{j}\left(v_{0}\right)} .
$$

By our assumptions, we have $0<\operatorname{Re}\left(\lambda_{j}(z)\right)<\frac{1}{2}$. From $\lambda_{j}(z)=\lambda_{j^{\prime}}(z)$, with $j, j^{\prime} \in$ $\left\{1, \ldots, m_{0}\right\}$ and $j \neq j^{\prime}$ it follows now that

$$
l_{j^{\prime}}\left(z-\frac{l_{j}(z)}{l_{j}\left(v_{0}\right)} v_{0}\right)=0 .
$$

In particular, we have $l_{j, j^{\prime}}(\operatorname{Re}(z))=0$. This is not possible, because $z \in T_{C_{1}}$.

Assume now that $x \in B_{1}$. We have, assuming that $l_{k}\left(v_{0}\right) \neq 0$, that

$$
\left|l_{k}\left(x+v_{0}\right)\right|=\left|l_{k}\left(v_{0}\right)\right| \cdot\left|\frac{l_{k}(x)}{l_{k}\left(v_{0}\right)}+1\right| \geq\left|l_{k}\left(v_{0}\right)\right| \cdot\left|-\frac{1}{2}+1\right|=\frac{1}{2}\left|l_{k}\left(v_{0}\right)\right| .
$$


If $l_{k}\left(v_{0}\right)=0$, then we have $l_{k}\left(x+v_{0}\right)=l_{k}(x)$.

Fix a $z \in T_{C_{1}}$. Then there exist some numbers $c_{1}(z) \geq 0, c_{2}(z) \geq 0$ such that for all $\lambda \in[0,1], \tau \in \mathbf{R}$ with $|\tau| \geq c_{1}(z)$ we have

$$
\begin{aligned}
& \left|f\left(z+\lambda v_{0}+i \tau v_{0}\right)\right| \\
& \quad=\left|g\left(z+\lambda v_{0}+i \tau v_{0}\right)\right| \cdot\left|\prod_{j=1}^{m}\left(1+\frac{1}{l_{j}\left(z+\lambda v_{0}+i \tau v_{0}\right)}\right)\right| \leq c_{2}(z) \kappa\left(z+[0,1] v_{0}\right) c\left(\tau v_{0}\right) .
\end{aligned}
$$

For any $z \in T_{C_{1}}$ we have therefore

$$
h_{C}(z)=-\sum_{j=1}^{m_{0}} \operatorname{Res}_{\lambda=\lambda_{j}(z)}\left(\lambda \rightarrow f\left(z+\lambda v_{0}\right)\right)+\frac{1}{2 \pi i} \int_{\operatorname{Re}(\lambda)=1} f\left(z+\lambda v_{0}\right) d \lambda .
$$

Since $\lambda_{j}(z) \neq \lambda_{j^{\prime}}(z)$ for $j, j^{\prime} \in\left\{1, \ldots, m_{0}\right\}, j \neq j^{\prime}, z \in T_{C_{1}}$, we have

$$
\begin{aligned}
\operatorname{Res}_{\lambda=\lambda_{j}(z)}\left(\lambda \rightarrow f\left(z+\lambda v_{0}\right)\right) & =\lim _{\lambda \rightarrow \lambda_{j}(z)}\left(\lambda-\lambda_{j}(z)\right) \prod_{k=1}^{m} \frac{l_{k}\left(z+\lambda v_{0}\right)+1}{l_{k}\left(z+\lambda v_{0}\right)} g\left(z+\lambda v_{0}\right) \\
& =\frac{1}{l_{j}\left(v_{0}\right)} g\left(z-\frac{l_{j}(z)}{l_{j}\left(v_{0}\right)} v_{0}\right) \prod_{1 \leq k \leq m, k \neq j} \frac{l_{j, k}(z)+1}{l_{j, k}(z)} .
\end{aligned}
$$

Put now for $j \in\left\{1, \ldots, m_{0}\right\}, z \in T_{B_{1}}$

$$
f_{j}(z):=-\frac{1}{l_{j}\left(v_{0}\right)} g\left(z-\frac{l_{j}(z)}{l_{j}\left(v_{0}\right)} v_{0}\right) \cdot \prod_{1 \leq k \leq m, k \neq j} \frac{l_{j, k}(z)+1}{l_{j, k}(z)}
$$

and

$$
g_{j}(z):=-\frac{1}{l_{j}\left(v_{0}\right)} \cdot g\left(z-\frac{l_{j}(z)}{l_{j}\left(v_{0}\right)} v_{0}\right) .
$$

Let $V_{1} \subset V$ be a hyperplane which does not contain $v_{0}$. We want to show that the function $f_{j}(z)$ is distinguished with respect to $\left(V_{1} ;\left(l_{j, k}\right)_{1 \leq k \leq m, k \neq j}\right)$. The function $f_{j}$ is meromorphic on $T_{B_{1}}$. Also, for all $x \in B_{1}$ and all $k \in\{1, \ldots, \hat{j}, \ldots, m\}$ we have $l_{j, k}(x)>-1$. Further, we have that

$$
g_{j}(z)=f_{j}(z) \cdot \prod_{1 \leq k \leq m, k \neq j} \frac{l_{j, k}(z)}{l_{j, k}(z)+1}
$$

is a holomorphic function on $T_{B_{1}}$. 
Let $\mathbf{K}_{1} \subset T_{B_{1}}\left(\subset T_{B}\right)$ be a compact, and let

$$
\mathbf{K}(j):=\left\{z-\frac{l_{j}(z)}{l_{j}\left(v_{0}\right)} v_{0} \mid z \in \mathbf{K}_{1}\right\} .
$$

This is a compact subset in $T_{B}$. Put

$$
\kappa_{j}\left(\mathbf{K}_{1}\right)=\frac{1}{\left|l_{j}\left(v_{0}\right)\right|} \kappa(\mathbf{K}(j)),
$$

where $\kappa(\mathbf{K}(j))$ is a constant such that $|g(z+i v)| \leq \kappa(\mathbf{K}(j)) c(v)$ for all $z \in \mathbf{K}(j)$ and all $v \in V$. For $v_{1} \in V_{1}$ we put

$$
c_{j}\left(v_{1}\right)=c\left(v_{1}-\frac{l_{j}\left(v_{1}\right)}{l_{j}\left(v_{0}\right)} v_{0}\right) .
$$

Then for all $z \in \mathbf{K}_{1}$ and $v \in V_{1}$ we have

$$
\left|g_{j}\left(z+i v_{1}\right)\right| \leq \kappa_{j}\left(\mathbf{K}_{1}\right) c_{j}\left(v_{1}\right) .
$$

Moreover, for any subspace $U_{1} \subset V_{1}$ and all $v_{1} \in V_{1}$ the function $U_{1} \rightarrow \mathbf{R}, u_{1} \rightarrow c_{j}\left(u_{1}+v_{1}\right)$ is measurable and we have

$$
c_{j, U_{1}}\left(v_{1}\right):=\int_{U_{1}} c_{j}\left(v_{1}+u_{1}\right) d u_{1}<\infty .
$$

For all $v_{1} \in V_{1}-U_{1}$ we have

$$
\lim _{\tau \rightarrow \pm \infty} c_{j, U_{1}}\left(\tau v_{1}\right)=0 .
$$

This shows that $f_{j}$ is distinguished with respect to $\left(V_{1} ;\left(l_{j, k}\right)_{1 \leq k \leq m, k \neq j}\right)$.

For $z \in T_{B_{1}}$ we put

$$
f_{0}(z):=\frac{1}{2 \pi i} \int_{\operatorname{Re}(\lambda)=1} f\left(z+\lambda v_{0}\right) d \lambda .
$$

If $l_{k}\left(v_{0}\right) \neq 0$ we have (as above) for all $x \in B_{1}$ the following inequality

$$
\left|l_{k}\left(x+v_{0}\right)\right| \geq \frac{1}{2}\left|l_{k}\left(v_{0}\right)\right| .
$$

Therefore, we conclude that the function

$$
\begin{aligned}
g_{0}(z) & :=f_{0}(z) \prod_{1 \leq k \leq m, l_{k}\left(v_{0}\right)=0} \frac{l_{k}(z)}{l_{k}(z)+1} \\
& =\frac{1}{2 \pi} \int_{-\infty}^{+\infty}\left\{\prod_{1 \leq k \leq m, l_{k}\left(v_{0}\right) \neq 0} \frac{l_{k}\left(z+v_{0}+i \tau v_{0}\right)+1}{l_{k}\left(z+v_{0}+i \tau v_{0}\right)}\right\} g\left(z+v_{0}+i \tau v_{0}\right) d \tau
\end{aligned}
$$


is holomorphic in $T_{B_{1}}$.

Further, we have for $z \in \mathbf{K}_{1}$ (with $\mathbf{K}_{1} \subset T_{B_{1}}$ a compact) and $v_{1} \in V_{1}$ the inequality

$$
\left|g_{0}\left(z+i v_{1}\right)\right| \leq \kappa_{0}\left(\mathbf{K}_{1}\right) c_{0}\left(v_{1}\right)
$$

where $\kappa_{0}\left(\mathbf{K}_{1}\right)$ is some suitable constant and

$$
c_{0}\left(v_{1}\right):=\int_{-\infty}^{+\infty} c\left(v_{1}+\tau v_{0}\right) d \tau .
$$

Again, for any subspace $U_{1} \subset V_{1}$ and any $u_{1} \in V_{1}$ we have that the map $U_{1} \rightarrow \mathbf{R}$ given by $u_{1} \rightarrow c_{0}\left(v_{1}+u_{1}\right)$ is measurable, and that

$$
c_{0, U_{1}}\left(v_{1}\right)=\int_{U_{1}} c_{0}\left(v_{1}+u_{1}\right) d u_{1}<\infty .
$$

For all $v_{1} \in V_{1}-U_{1}$ we have

$$
\lim _{\tau \rightarrow \pm \infty} c_{0, U_{1}}\left(\tau v_{1}\right)=0 .
$$

Therefore, $f_{0}$ is distinguished with respect to $\left(V_{1} ;\left(l_{k}\right)_{1 \leq k \leq m, l_{k}\left(v_{0}\right) \neq 0}\right)$.

The Cauchy-Riemann equations imply that $g_{0}$ is invariant under $\mathbf{C} v_{0}$, that is, for all $z_{1}, z_{2} \in T_{B_{1}}$ with $z_{1}-z_{2} \in \mathbf{C} v_{0}$ we have $g_{0}\left(z_{1}\right)=g_{0}\left(z_{2}\right)$. We see that $f_{0}$ is also invariant under $\mathbf{C} v_{0}$ (in this sense), as well as $f_{1}, \ldots, f_{m_{0}}$ (this can be seen from the explicit representation of these functions). For $z \in T_{C_{1}}$ we have

$$
h_{C}(z)=f_{0}(z)+\sum_{j=1}^{m_{0}} f_{j}(z)=\sum_{j=0}^{m_{0}} f_{j}(z) .
$$

Moreover, for such $z$ we have

$$
\tilde{f}_{C}(z)=\frac{1}{(2 \pi)^{d-1}} \int_{V_{1}} h_{C}\left(z+i v_{1}\right) d v_{1}=\sum_{j=0}^{m_{0}} \frac{1}{(2 \pi)^{d-1}} \int_{V_{1}} f_{j}\left(z+i v_{1}\right) d v_{1},
$$

where $d v_{1} d \tau=d v\left(\right.$ and $\left.\operatorname{Vol}_{d \tau}\left(\left\{\lambda v_{0} \mid \lambda \in[0,1]\right\}\right)=1\right)$.

By our induction hypothesis, there exists an open and convex neighborhood $B^{\prime}$ of $\mathbf{0}$ in $E$ and linear forms $\tilde{l}_{1}, \ldots, \tilde{l}_{\tilde{m}}$, which vanish on $V$, such that

$$
\tilde{f}_{C}(z) \prod_{j=1}^{\tilde{m}} \tilde{l}_{j}(z)
$$


has a holomorphic continuation to $T_{B^{\prime}}$. (Strictly speaking, from the induction hypothesis it follows only that the linear forms $\tilde{l}_{1}, \ldots, \tilde{l}_{\tilde{m}} \in E^{\vee}$ vanish on $V_{1}$. But since the functions $f_{0}, \ldots, f_{m_{0}}$ "live" already on a tube domain in $\left(E / \mathbf{R} v_{0}\right)_{\mathbf{C}}$, it follows that the linear forms are also $\mathbf{R} v_{0}$-invariant.)

Now we notice that $\tilde{f}_{C}(z) \prod_{j=1}^{\tilde{m}} \tilde{l}_{j}(z)$ is holomorphic on $T_{C}$. Let $\tilde{B}$ be the convex hull of $B^{\prime} \cup C$. Then we have that

$$
\tilde{f}_{C}(z) \prod_{j=1}^{\tilde{m}} \tilde{l}_{j}(z)
$$

is holomorphic on $T_{\tilde{B}}$ (cf. [18], Theorem 2.5.10).

7.4 Let $E, V, l_{1}, \ldots, l_{m}$ and $B \subset\left\{x \in E \mid l_{j}(x)>-1 \forall j=1, \ldots, m\right\}$ be as above. Let $C$ be a connected component of $B-\bigcup_{j=1}^{m} H_{j}$. Let $f \in \mathcal{M}\left(T_{B}\right)$ be a distinguished function with respect to $\left(V ; l_{1}, \ldots, l_{m}\right)$.

Proposition 7.4 There exist an open convex neighborhood $\tilde{B}$ of $\mathbf{0}$ in $E$ containing $C$ and linear forms $\tilde{l}_{1}, \ldots, \tilde{l}_{\tilde{m}} \in E^{\vee}$ vanishing on $V$ such that

a) for all $j \in\{1, \ldots, \tilde{m}\}$ we have $\operatorname{Ker}\left(\tilde{l}_{j}\right) \cap C=\emptyset$,

b) $\tilde{f}_{C}(z) \prod_{j=1}^{\tilde{m}} \tilde{l}_{j}(z)$ has a holomorphic continuation to $T_{\tilde{B}}$.

Proof. By the proposition above, there exist linear forms $\tilde{l}_{1}, \ldots, \tilde{l}_{\tilde{m}}$ such that $V \subset$ $\cap_{j=1}^{\tilde{m}} \operatorname{Ker}\left(\tilde{l}_{j}\right)$ and $\tilde{f}_{C}(z) \prod_{j=1}^{\tilde{m}} \tilde{l}_{j}(z)$ has a holomorphic continuation to a tube domain $T_{\tilde{B}}$ over a convex open neighborhood $\tilde{B}$ of $\mathbf{0} \in E$ containing $C$. Suppose that there exist an $x_{0} \in C$ and a $j_{0} \in\{1, \ldots, \tilde{m}\}$ such that $\tilde{l}_{j_{0}}\left(x_{0}\right)=0$. Then the function

$$
\tilde{f}_{C}(z) \prod_{j \neq j_{0}} \tilde{l}_{j}(z)
$$

is still holomorphic in $T_{\tilde{B}^{\prime}}$ with $\tilde{B}^{\prime}=\left(B-\operatorname{Ker}\left(\tilde{l}_{j_{0}}\right)\right) \cup C$. It is easy to see that $\tilde{B}^{\prime}$ is connected. The convex hull of $\tilde{B}^{\prime}$ is equal to $\tilde{B}$. Therefore, already the function

$$
\tilde{f}_{C}(z) \prod_{j \neq j_{0}} \tilde{l}_{j}(z)
$$

is holomorphic on $T_{\tilde{B}}$ (cf. [18], loc. cit.). 
7.5 As above, let $E$ be a finite dimensional vector space over $\mathbf{R}$ and let $l_{1}, \ldots, l_{m}$ be linearly independent linear forms on $E$. Put $H_{j}:=\operatorname{Ker}\left(l_{j}\right)$, for $j=1, \ldots, m, E^{(0)}=$ $\bigcap_{j=1}^{m} H_{j}$ and $E_{0}=E / E^{(0)}$. Let $\pi_{0}: E \rightarrow E_{0}$ be the canonical projection. Let $V \subset E$ be a subspace with $V \cap E^{(0)}=\{\boldsymbol{0}\}$, such that $\left.\pi_{0}\right|_{V}: V \rightarrow E_{0}$ is an injective map. Let

$$
E_{0}^{+}:=\left\{x \in E_{0} \mid l_{j}(x) \geq 0, j=1, \ldots, m\right\}
$$

and let $\psi: E_{0} \rightarrow P:=E_{0} / \pi_{0}(V)$ be the canonical projection. We want to assume that $\pi_{0}(V) \cap E_{0}^{+}=\{\mathbf{0}\}$, so that $\Lambda:=\psi\left(E_{0}^{+}\right)$is a strictly convex polyhedral cone.

Let $d y$ be the Haar measure on $E_{0}^{\vee}$ normalized by $\operatorname{Vol}_{d y}\left(E_{0}^{\vee} / \oplus_{j=1}^{m} \mathbf{Z} l_{j}\right)=1$. Let $A \subset V$ be a lattice, and let $d v$ be a measure on $V$ normalized by $\operatorname{Vol}_{d v}(V / A)=1$. On $V^{\vee}$ we have a measure $d y^{\prime}$ normalized by $A^{\vee}$ and a section of the projection $E_{0}^{\vee} \rightarrow V^{\vee}$ gives a measure $d y^{\prime \prime}$ on $P^{\vee}$ with $d y=d y^{\prime} d y^{\prime \prime}$.

Let $B \subset E$ be an open and convex neighborhood of $\mathbf{0}$, such that for all $x \in B$ and $j \in\{1, \ldots, m\}$ we have $l_{j}(x)>-1$. Let $f \in \mathcal{M}\left(T_{B}\right)$ be a meromorphic function in the tube domain over $B$ which is distinguished with respect to $\left(V ; l_{1}, \ldots, l_{m}\right)$. Put

$$
\begin{gathered}
B^{+}=B \cap\left\{x \in E \mid l_{j}(x)>0, j=1, \ldots, m\right\}, \\
\tilde{f}_{B^{+}}(z)=\frac{1}{(2 \pi)^{d}} \int_{V} f(z+i v) d v
\end{gathered}
$$

where $d=\operatorname{dim} V$.

By 7.3, the function $\tilde{f}_{B^{+}}: T_{B^{+}} \rightarrow \mathbf{C}$ is holomorphic and it has a meromorphic continuation to a neighborhood of $\mathbf{0} \in E_{\mathbf{C}}$. Put

$$
g(z)=f(z) \prod_{j=1}^{m} \frac{l_{j}(z)}{l_{j}(z)+1} .
$$

Proposition 7.5 For $x_{0} \in B^{+}$we have

$$
\lim _{s \rightarrow 0} s^{m-d} \tilde{f}_{B^{+}}\left(s x_{0}\right)=g(\mathbf{0}) \mathcal{X}\left(\psi\left(x_{0}\right)\right) .
$$

Proof. For $j \in J:=\{1, \ldots, m\}$ we define

$$
\begin{gathered}
H_{j,+}:=\left\{v \in V \mid l_{j}(v)=1\right\}, \\
H_{j,-}:=\left\{v \in V \mid l_{j}(v)=-1\right\} .
\end{gathered}
$$


Let $\mathcal{C}$ be the set of connected components of $V-\bigcup_{j=1}^{m}\left(H_{j,+} \cup H_{j,-}\right)$. For a $C \in \mathcal{C}$ we put

$$
J_{C}:=\left\{j \in J|| l_{j}(v) \mid<1 \text { for all } v \in C\right\}
$$

and

$$
V^{C}:=V \cap \bigcap_{j \in J_{C}} H_{j}
$$

Denote by $V_{C}$ the complement to $V^{C}$ in $V$ and let $\pi_{C}: V=V_{C} \oplus V^{C} \rightarrow V_{C}$ be the projection. Since the map $V_{C} \rightarrow \mathbf{R}^{J_{C}}, v \rightarrow\left(l_{j}(v)\right)_{j \in J_{C}}$, is injective we see that $\pi_{C}(C)$ is a bounded open subset of $V_{C}$. For $v_{1} \in \pi_{C}(C)$ we put

$$
C\left(v_{1}\right):=\left\{v^{\prime} \in V^{C} \mid v_{1}+v^{\prime} \in C\right\} .
$$

The set $C\left(v_{1}\right)$ is a convex open subset of $V^{C}$. Let $d v_{1}, d v^{\prime}$ be measures on $V_{C}$ (resp. on $\left.V^{C}\right)$, with $d v_{1} d v^{\prime}=d v$. For all $s \in(0,1]$ we have

$$
\begin{gathered}
s^{m-d} \int_{C} f\left(s x_{0}+i v\right) d v=s^{m-d} \int_{\pi_{C}(C)} \int_{C\left(v_{1}\right)} f\left(s x_{0}+i v_{1}+i v^{\prime}\right) d v^{\prime} d v_{1} \\
=s^{m-d^{C}} \int_{\frac{1}{s} \pi_{C}(C)} \int_{C\left(s v_{1}\right)} f\left(s x_{0}+i s v_{1}+i v^{\prime}\right) d v^{\prime} d v_{1} \\
=\int_{V} \chi_{C_{s}}(v) s^{m-d^{C}} f_{C, s}(v) d v .
\end{gathered}
$$

Here we denoted by $d^{C}:=\operatorname{dim} V^{C}$ and by

$$
C_{s}:=\left\{v=v_{1}+v^{\prime} \in V_{C} \oplus V^{C} \mid s v_{1}+v^{\prime} \in C\right\}
$$

and by $\chi_{C_{s}}$ the set-theoretic characteristic function of $C_{s}$. We have put for any $s \in(0,1]$ and any $v=v_{1}+v^{\prime} \in V$

$$
f_{C, s}(v):=f\left(s x_{0}+i s v_{1}+i v^{\prime}\right) .
$$

The set

$$
\mathbf{K}_{C}:=\left\{s x_{0}+i v_{1} \mid s \in[0,1], v_{1} \in \overline{\pi_{C}(C)}\right\}
$$

is contained in $T_{B}$ and is compact. Further, there exist $c^{\prime}, c^{\prime \prime} \geq 0$ such that for all $s \in[0,1]$ and all $v=v_{1}+v^{\prime} \in C$ we have

$$
\left|\prod_{j \in J_{C}}\left(l_{j}\left(s x_{0}+i v\right)+1\right) \prod_{j \notin J_{C}} \frac{l_{j}\left(s x_{0}+i v\right)+1}{l_{j}\left(s x_{0}+i v\right)}\right| \leq c^{\prime},
$$




$$
1 \leq c^{\prime \prime} \cdot\left|\prod_{j \notin J_{C}} \frac{1}{l_{j}\left(x_{0}+i v_{1}\right)}\right|
$$

Therefore, for $s \in(0,1]$ and $v \in V$ we have

$$
\begin{gathered}
\left|\chi_{C_{s}}(v) s^{m-d^{C}} f_{C, s}(v)\right| \\
=\left|\chi_{C_{s}}(v) g\left(s x_{0}+i s v_{1}+i v^{\prime}\right) s^{m-d^{C}} \prod_{j \in J}\left(1+\frac{1}{l_{j}\left(s x_{0}+i s v_{1}+i v^{\prime}\right)}\right)\right| \\
\leq \chi_{C_{s}}(v) s^{m-d^{C}}\left|\prod_{j \in J_{C}} \frac{1}{l_{j}\left(s x_{0}+i s v_{1}\right)} \prod_{j \notin J_{C}} \frac{1}{l_{j}\left(x_{0}+i v_{1}\right)}\right| c^{\prime} c^{\prime \prime} \kappa\left(\mathbf{K}_{C}\right) c\left(v^{\prime}\right) \\
\leq c^{\prime} c^{\prime \prime} \kappa\left(\mathbf{K}_{C}\right)\left|\prod_{j \in J} \frac{1}{l_{j}\left(x_{0}+i v_{1}\right)}\right| c\left(v^{\prime}\right) s^{m-d^{C}-\# J_{C}} \\
\leq c^{\prime} c^{\prime \prime} \kappa\left(\mathbf{K}_{C}\right)\left|\prod_{j \in J} \frac{1}{l_{j}\left(x_{0}+i v_{1}\right)}\right| c\left(v^{\prime}\right),
\end{gathered}
$$

since $m-d^{C}-\# J_{C} \geq 0$. (The constant $\kappa\left(\mathbf{K}_{C}\right)$ and the function $c: V \rightarrow \mathbf{R}_{\geq 0}$ were introduced above.)

The $\mathcal{X}$-function corresponding to the cone $E_{0}^{+} \subset E_{0}$ (and the measure $d y$ ) is given by

$$
\mathcal{X}_{E_{0}^{+}}\left(x_{0}+i v_{1}\right)=\prod_{j=1}^{m} \frac{1}{l_{j}\left(x_{0}+i v_{1}\right)} .
$$

Since the map from $V_{C}$ to $E_{0}$ is injective and since $\pi_{0}\left(V_{C}\right) \cap E_{0}^{+}=\{\mathbf{0}\}$ we know that the function $v_{1} \rightarrow \mathcal{X}_{E_{0}^{+}}\left(x_{0}+i v_{1}\right)$ is absolutely integrable over $V_{C}$ (by 7.2). Therefore,

$$
v=v_{1}+v^{\prime} \mapsto c^{\prime} c^{\prime \prime} \kappa\left(\mathbf{K}_{C}\right)\left|\prod_{j \in J} \frac{1}{l_{j}\left(x_{0}+i v_{1}\right)}\right| c\left(v^{\prime}\right)
$$

is integrable over $V$.

For a fixed $v \in V$ we consider the limit

$$
\lim _{s \rightarrow 0} \chi_{C_{s}}(v) s^{m-d^{C}} f_{C, s}(v) .
$$


The estimate above shows that this limit is 0 if $m-d^{C}-\# J_{C}>0$. Therefore, we assume that $m=d^{C}+\# J_{C}$. Then the map $V^{C} \rightarrow \mathbf{R}^{J-J_{C}}$ is an isomorphism. Since $\pi_{0}(V) \cap E_{0}^{+}=\{\mathbf{0}\}$, it follows that $J_{C}=J$. There exists exactly one $C \in \mathcal{C}$ with $J_{C}=J$ and we denote it by $C^{\circ}$. This $C^{\circ}$ contains $\mathbf{0}$ and for all sufficiently small $s>0$ we have $s \cdot v \in C^{\circ}$, and therefore, $v \in C_{s}^{\circ}$. Moreover, we have

$$
\begin{gathered}
\lim _{s \rightarrow 0} s^{m} f_{C, s}(v)=\lim _{s \rightarrow 0} s^{m} g\left(s x_{0}+i s v\right) \prod_{j=1}^{m} \frac{l_{j}\left(s x_{0}+i s v\right)+1}{l_{j}\left(s x_{0}+i s v\right)} \\
=g(\mathbf{0}) \prod_{j=1}^{m} \frac{1}{l_{j}\left(x_{0}+i v\right)} .
\end{gathered}
$$

Using the theorem of dominated convergence (Lebesgue's theorem), we obtain

$$
\begin{aligned}
\lim _{s \rightarrow 0} s^{m-d} \tilde{f}_{B^{+}}\left(s x_{0}\right) & =\lim _{s \rightarrow 0} \sum_{C \in \mathcal{C}} \frac{1}{(2 \pi)^{d}} \int_{V} \chi_{C_{s}}(v) s^{m-d} f_{C, s}(v) d v \\
& =\frac{1}{(2 \pi)^{d}} g(\mathbf{0}) \int_{V} \prod_{j=1}^{m} \frac{1}{l_{j}\left(x_{0}+i v\right)} d v \\
& =g(\mathbf{0}) \mathcal{X}_{\Lambda}\left(\psi\left(x_{0}\right)\right) .
\end{aligned}
$$

7.6 In this section we will prove an assertion made in the proof of Theorem 5.5, namely that a certain function is sufficient in the sense of 7.3. More precisely, let $V$ be a $d$ dimensional vector space over $\mathbf{R}$ and let $\left(l_{1}, \ldots, l_{d}\right)$ be a basis of $V^{\vee}=\operatorname{Hom}_{\mathbf{R}}(V, \mathbf{R})$. Fix some $\epsilon>0$. Define

$$
c: V \rightarrow \mathbf{R}_{\geq 0}
$$

by

$$
c(v)=\prod_{j=1}^{d} \frac{1}{\left(1+\left|l_{j}(v)\right|\right)^{1+\epsilon}} .
$$

We want to show that $c$ is a sufficient function in the sense of 7.3. The following fact is elementary:

Fact. There exists a $\kappa_{1}=\kappa_{1}(\epsilon)>0$ such that for all $\xi \geq 0$

$$
\int_{0}^{\xi} \frac{d t}{((1+\xi-t)(1+t))^{1+\epsilon}} \leq \frac{\kappa_{1}}{(1+\xi)^{1+\epsilon}} .
$$


Lemma. There exists a $\kappa_{2}=\kappa_{2}(\epsilon)>0$ such that for all $x_{1} \leq x_{2} \leq \ldots \leq x_{n}$ we have

$$
\begin{gathered}
\kappa_{2} \int_{-\infty}^{+\infty} \frac{d t}{\prod_{j=1}^{n}\left(1+\left|t+x_{j}\right|\right)^{1+\epsilon}} \leq \prod_{j=2}^{n} \frac{1}{\left(1+x_{j}-x_{1}\right)^{1+\epsilon}} \\
+\sum_{r=2}^{n}\left(\left(\prod_{j=1}^{r-2} \frac{1}{1+x_{r-2}-x_{j}}\right) \cdot \frac{1}{1+x_{r}-x_{r-1}} \cdot\left(\prod_{j=r+1}^{n} \frac{1}{1+x_{j}-x_{r}}\right)\right)^{1+\epsilon} \\
+\prod_{j=1}^{n-1} \frac{1}{\left(1+x_{n}-x_{j}\right)^{1+\epsilon}} .
\end{gathered}
$$

Proof. We divide the integral over $\mathbf{R}$ into integrals over $\left(-x_{1}, \infty\right),\left(-\infty,-x_{n}\right)$ and $\left(-x_{r},-x_{r-1}\right), r=2, \ldots, n$. We have

$$
\int_{-x_{1}}^{\infty} \frac{d t}{\prod_{j=1}^{n}\left(1+\left|t+x_{j}\right|\right)^{1+\epsilon}} \leq \int_{0}^{\infty} \frac{d t}{(1+t)^{1+\epsilon}} \cdot \prod_{j=2}^{n} \frac{1}{\left(1+x_{j}-x_{1}\right)^{1+\epsilon}}
$$

and

$$
\int_{-\infty}^{-x_{n}} \frac{d t}{\prod_{j=1}^{n}\left(1+\left|t+x_{j}\right|\right)^{1+\epsilon}} \leq \int_{0}^{\infty} \frac{d t}{(1+t)^{1+\epsilon}} \cdot \prod_{j=1}^{n} \frac{1}{\left(1+x_{n}-x_{j}\right)^{1+\epsilon}} .
$$

Moreover, for $r=2, \ldots, n$ :

$$
\begin{gathered}
\int_{-x_{r}}^{-x_{r-1}} \frac{d t}{\prod_{j=1}^{n}\left(1+\left|t+x_{j}\right|\right)^{1+\epsilon}} \\
=\int_{0}^{x_{r}-x_{r-1}} \frac{d t}{\prod_{j=1}^{r-1}\left(1+x_{r}-x_{j}-t\right)^{1+\epsilon} \cdot \prod_{j=r}^{n}\left(1+x_{j}-x_{r}+t\right)^{1+\epsilon}} \\
\leq \prod_{j=1}^{r-2} \frac{1}{\left(1+x_{r-1}-x_{j}\right)^{1+\epsilon}} \prod_{j=r+1}^{n} \frac{1}{\left(1+x_{j}-x_{r}\right)^{1+\epsilon}} \int_{0}^{x_{r}-x_{r-1}} \frac{d t}{\left(\left(1+x_{r}-x_{r-1}-t\right)(1+t)\right)^{1+\epsilon}} .
\end{gathered}
$$

The fact recalled above gives us an estimate of the integral in the last expression. This completes the proof.

Proposition 7.6 Let $V,\left(l_{1}, \ldots, l_{d}\right), \epsilon$ and $c$ be as above. Let $U \subset V$ be a subspace and $d u$ a Lebesgue measure on $U$. Then there exist a $\kappa=\kappa(\epsilon, U, d u)>0$ and a finite family $\left\{\left(l_{\alpha, 1}, \ldots, l_{\alpha, d^{\prime}}\right)\right\}_{\alpha \in A}$ of basis' of $(V / U)^{\vee}\left(d^{\prime}=\operatorname{dim} V / U\right)$ such that for all $v \in V$ we have

$$
\int_{U} c(v+u) d u \leq \kappa \sum_{\alpha \in A} \frac{1}{\prod_{j=1}^{d^{\prime}}\left(1+\left|l_{\alpha, j}(v)\right|\right)^{1+\epsilon}} .
$$


Proof. The proof proceeds by induction on $\operatorname{dim} U$. For $\operatorname{dim} U=0$ there is nothing to show. Let $\operatorname{dim} U>0$. Choose $u \in U-\{\mathbf{0}\}$ and a splitting $U=U_{1} \oplus \mathbf{R} u$ with $U_{1}$ of codimension one. By induction hypothesis there exist a $\kappa^{\prime}>0$ and a finite family $\left\{\left(l_{\alpha, 1}, \ldots, l_{\alpha, d^{\prime}+1}\right)\right\}_{\alpha \in A^{\prime}}$ of basis' of $\left(V / U_{1}\right)^{\vee}$ such that for all $v \in V$

$$
\int_{U} c(v+u) d u \leq \kappa^{\prime} \sum_{\alpha \in A^{\prime}} \int_{-\infty}^{+\infty} \frac{d t}{\prod_{j=1}^{d^{\prime}+1}\left(1+\left|l_{\alpha, j}(v)+t l_{\alpha, j}(u)\right|\right)^{1+\epsilon}} .
$$

Now it is easy to see how to use the Lemma above to get the asserted inequality.

\section{Some statements on Eisenstein series}

8.1 Let $G$ be a semi-simple simply connected algebraic group which is defined and split over $F$. Fix a Borel subgroup $P_{0}$ (defined over $F$ ) and a Levi decomposition $P_{0}=S_{0} U_{0}$, where $S_{0}$ is a maximal $F$-rational torus of $G$. Denote by $\mathbf{g}$ (resp. $\mathbf{a}_{0}$ ) the Lie algebra of $G\left(\right.$ resp. $\left.S_{0}\right)$.

We are going to define a certain maximal compact subgroup $\mathbf{K}_{G} \subset G(\mathbf{A})$. This maximal compact subgroup will have the advantage that the constant term of Eisenstein series, more precisely, certain intertwining operators, can be calculated explicitly, uniformly with respect to all places of $F$. In general, i.e., for an arbitrary maximal compact subgroup, there will be some places where such an explicit expression is not available. In principle, this should cause no problems. Any statement in this section should be valid for an arbitrary maximal compact subgroup.

8.2 Let $\Phi=\Phi\left(G, S_{0}\right)$ be the root system of $G$ with respect to $S_{0}$. We denote by $\Delta_{0}$ the basis of simple roots determined by $P_{0}$. For $\alpha \in \Phi$ let

$$
\mathbf{g}_{\alpha}:=\{X \in \mathbf{g} \mid[H, X]=\alpha(H) X\}
$$

be the corresponding root space.

Let $\left(\left(H_{\alpha}\right)_{\alpha \in \Delta_{0}},\left(X_{\alpha}\right)_{\alpha \in \Phi}\right)$ be the Chevalley basis of $\mathbf{g}$. In particular, this means that

$$
\begin{gathered}
\mathbf{g}_{\alpha}=F X_{\alpha}(\alpha \in \Phi), \quad\left[X_{\alpha}, X_{-\alpha}\right]=H_{\alpha}\left(\alpha \in \Delta_{0}\right), \\
\mathbf{a}_{0}=\oplus_{\alpha \in \Delta_{0}} F H_{\alpha} .
\end{gathered}
$$


Put

$$
\mathrm{g}_{\mathbf{Q}}=\sum_{\alpha \in \Delta_{0}} \mathrm{Q} H_{\alpha}+\sum_{\alpha \in \Phi} \mathrm{Q} X_{\alpha} \subset \mathbf{g} .
$$

This is a Q-structure for $\mathbf{g}$ and for any $v \in \operatorname{Val}(F)$ the Lie algebra of $G\left(F_{v}\right)$ is $\mathbf{g} \otimes_{\mathbf{Q}} F_{v}$. We put

$$
\begin{gathered}
\mathbf{k}:=\oplus_{\alpha \in \Phi^{+}} \mathbf{R}\left(X_{\alpha}-X_{-\alpha}\right), \\
\mathbf{p}:=\bigoplus_{\alpha \in \Delta_{0}} \mathbf{R} H_{\alpha} \oplus \bigoplus_{\alpha \in \Phi^{+}} \mathbf{R}\left(X_{\alpha}+X_{-\alpha}\right),
\end{gathered}
$$

where $\Phi^{+}$is the set of positive roots of $\Phi$ determined by $\Delta_{0}$. Then $\mathbf{k} \oplus \mathbf{p}$ is a Cartan decomposition of $\mathbf{g}_{\mathbf{Q}} \otimes_{\mathbf{Q}} \mathbf{R}, \mathbf{g}_{c}:=\mathbf{k} \oplus i \mathbf{p} \subset \mathbf{g}_{\mathbf{Q}} \otimes_{\mathbf{Q}} \mathbf{C}$ is a compact form of $\mathbf{g}_{\mathbf{Q}} \otimes_{\mathbf{Q}} \mathbf{C}$ and $\mathbf{g}_{\mathbf{Q}} \otimes_{\mathbf{Q}} \mathbf{C}=\mathbf{g}_{c} \oplus i \mathbf{g}_{c}$ is a Cartan decomposition of $\mathbf{g}_{\mathbf{Q}} \otimes_{\mathbf{Q}} \mathbf{C}$.

For any complex place $v$ of $F$, we define $\mathbf{K}_{v}$ to be $\left\langle\exp \left(\mathbf{g}_{c}\right)\right\rangle \subset G\left(F_{v}\right)$ (identifying $F_{v}$ with $\mathbf{C}$ via a corresponding embedding $F \hookrightarrow \mathbf{C}$ ). If $v$ is a real place of $F$ we define $\mathbf{K}_{v}=$ $G\left(F_{v}\right) \cap\left\langle\exp \left(\mathbf{g}_{c}\right)\right\rangle$ (identifying $F_{v}(\sqrt{-1}) \simeq \mathbf{C}$ via a corresponding embedding $F \hookrightarrow \mathbf{R}$ ). In this case, $\mathbf{K}_{v}$ contains $\langle\exp (\mathbf{k})\rangle$. In both cases $\mathbf{K}_{v}$ is a maximal compact subgroup of $G\left(F_{v}\right)$.

Now let $v$ be a finite place of $F$ and let $\mathbf{K}_{v}$ be the stabilizer of the lattice

$$
\sum_{\alpha \in \Delta_{0}} \mathcal{O}_{v} \cdot H_{\alpha}+\sum_{\alpha \in \Phi} \mathcal{O}_{v} \cdot X_{\alpha} \subset \mathbf{g} \otimes_{F} F_{v}
$$

By [10], sec. 3, Example 2, $\mathbf{K}_{v}$ is a maximal compact subgroup of $G\left(F_{v}\right)$. In any case, the Iwasawa decomposition $G\left(F_{v}\right)=P_{0}\left(F_{v}\right) \mathbf{K}_{v}$ holds (for non-archimedean $v$, cf. [10], loc. cit.). Then $\mathbf{K}_{G}=\prod_{v} \mathbf{K}_{v}$ is a maximal compact subgroup of $G(\mathbf{A})$ and $G(\mathbf{A})=P_{0}(\mathbf{A}) \mathbf{K}_{G}$.

8.3 As in section 2.3 we defined for any standard parabolic subgroup $P \subset G$

$$
H_{P}=H_{P, \mathbf{K}_{G}}: G(\mathbf{A}) \rightarrow \operatorname{Hom}_{\mathbf{C}}\left(X^{*}(P)_{\mathbf{C}}, \mathbf{C}\right)
$$

by $\left\langle\lambda, H_{P}(g)\right\rangle=\log \left(\prod_{v}\left|\lambda\left(p_{v}\right)\right|_{v}\right)$ for $\lambda \in X^{*}(P)$ and $g=p k, p=\left(p_{v}\right)_{v} \in P(\mathbf{A}), k \in \mathbf{K}_{G}$.

The restriction of $H_{P_{0}}$ to $S_{0}(\mathbf{A})$ is a homomorphism, its kernel will be denoted by $S_{0}(\mathbf{A})^{1}$. The choice of a projection $\mathbf{G}_{m}(\mathbf{A}) \rightarrow \mathbf{G}_{m}(\mathbf{A})^{1}$ induces by means of an isomorphism $S_{0} \rightarrow \mathbf{G}_{m, F}^{\# \Delta_{0}}$ a projection $S_{0}(\mathbf{A}) \rightarrow S_{0}(\mathbf{A})^{1}$ and this in turn gives an embedding

$$
\mathcal{U}_{0}:=\left(S_{0}(\mathbf{A})^{1} / S_{0}(F)\left(S_{0}(\mathbf{A}) \cap \mathbf{K}_{G}\right)\right)^{*} \hookrightarrow\left(S_{0}(\mathbf{A}) / S_{0}(F)\left(S_{0}(\mathbf{A}) \cap \mathbf{K}_{G}\right)\right)^{*}
$$


Let $\left(\varpi_{\alpha}\right)_{\alpha \in \Delta_{0}}$ be the basis of $X^{*}\left(S_{0}\right)$ which is determined by $\left\langle\varpi_{\alpha}, \beta\right\rangle=\delta_{\alpha \beta}$ for all $\alpha, \beta \in$ $\Delta_{0}$. Let $P \subset G$ be a standard parabolic subgroup. Then $\varpi_{\alpha}$ for all $\alpha \in \Delta_{P}$ lifts to a character of $P$ and $\left(\varpi_{\alpha}\right)_{\alpha \in \Delta_{P}}$ is a basis of $X^{*}(P)$. Put

$$
\mathcal{U}_{P}:=\left\{\chi \mid \chi=\chi_{0} \circ \prod_{\alpha \in \Delta_{P}} \check{\alpha} \circ \varpi_{\alpha} \text { with } \chi_{0} \in \mathcal{U}_{0}\right\} .
$$

Any $\chi \in \mathcal{U}_{P}$ is a character of $P(\mathbf{A}) / P(F)\left(P(\mathbf{A}) \cap \mathbf{K}_{G}\right)$. Define

$$
\phi_{\chi}: G(\mathbf{A}) \rightarrow S^{1}
$$

by $\phi_{\chi}(p k)=\chi(p)$ for $p \in P(\mathbf{A}), k \in \mathbf{K}_{G}$. The Eisenstein series

$$
E_{P}^{G}(\lambda, \chi, g)=\sum_{g \in P(F) \backslash G(F)} \phi_{\chi}(\gamma g) e^{\left\langle\lambda+\rho_{P}, H_{P}(\gamma g)\right\rangle}
$$

converges absolutely and uniformly for $\operatorname{Re}(\lambda)$ contained in any compact subset of the open cone $\rho_{P}+X^{*}(P)^{+}$(cf. [16], Théorème III) and can be continued meromorphically to the whole of $X^{*}(P)_{\mathbf{C}}$.

For the Eisenstein series corresponding to $P_{0}$ a proof is given in [27], chapitre IV. In section 8.4 we will give an explicit expression for the Eisenstein series $E_{P}^{G}$, with $P \neq P_{0}$ as an iterated residue of $E_{P_{0}}^{G}$ which shows the claimed meromorphy on $X^{*}(P)_{\mathbf{C}}$.

Let $\chi \in \mathcal{U}_{0}$. The constant term of $E_{P_{0}}^{G}(\lambda, \chi)$ along $P=L U$ is by definition

$$
E_{P_{0}}^{G}(\lambda, \chi)_{P}(g)=\int_{U(F) \backslash U(\mathbf{A})} E_{P_{0}}^{G}(\lambda, \chi, u g) d u,
$$

where the Haar measure on $U(\mathbf{A})$ is normalized such that $U(F) \backslash U(\mathbf{A})$ gets volume one. It is an elementary calculation to show that for any parabolic subgroup $P \nsupseteq P_{0}$ the constant term $E_{P_{0}}^{G}(\lambda, \chi)_{P}$ is orthogonal to all cusp forms in $A_{0}(L(F) U(\mathbf{A}) \backslash G(\mathbf{A})$ ) (cf. [27], I.2.18, for the definition of this space). More precisely, for any parabolic subgroup $P \nsupseteq P_{0}$ the cuspidal component of $E_{P_{0}}^{G}(\lambda, \chi)$ along $P$ vanishes (cf. [27], I.3.5, for the definition of "cuspidal component").

By Lemme I.4.10 in [27], the singularities of the Eisenstein series $E_{P_{0}}^{G}(\lambda, \chi)$ and the singularities of $E_{P_{0}}^{G}(\lambda, \chi)_{P_{0}}$ coincide. Let

$$
\mathcal{W}=\operatorname{Norm}_{G(F)}\left(S_{0}(F)\right) / S_{0}(F)
$$

be the Weyl group of $G$ with respect to $S_{0}$. For any $w \in \mathcal{W}$ we normalize the Haar measures such that

$$
\int_{\left(U_{0}(F) \cap w U_{0}(F) w^{-1}\right) \backslash\left(U_{0}(\mathbf{A}) \cap w U_{0}(\mathbf{A}) w^{-1}\right)} d u=1
$$


and on $\left(U_{0}(\mathbf{A}) \cap w U_{0}(\mathbf{A}) w^{-1}\right) \backslash U_{0}(\mathbf{A})$ we take the quotient measure. Using Bruhat's decomposition $G(F)=\bigcup_{w \in \mathcal{W}} P_{0}(F) w^{-1} P_{0}(F)$ we can calculate

$$
\int_{U_{0}(F) \backslash U_{0}(\mathbf{A})} E_{P_{0}}^{G}(\lambda, \chi, u g) d u=\sum_{w \in \mathcal{W}} c(w, \lambda, \chi) \phi_{w \chi}(g) e^{\left\langle w \lambda+\rho_{0}, H_{P_{0}}(g)\right\rangle},
$$

where $\rho_{0}=\rho_{P_{0}},(w \chi)(t)=\chi\left(w^{-1} t w\right)$ for all $t \in S_{0}(\mathbf{A})$, and the functions $c(w, \lambda, \chi)$ are given by

$$
c(w, \lambda, \chi):=\int_{\left(U_{0}(\mathbf{A}) \cap w U_{0}(\mathbf{A}) w^{-1}\right) \backslash U_{0}(\mathbf{A})} \phi_{\chi}\left(w^{-1}\right) e^{\left\langle\lambda+\rho_{P}, H_{P_{0}}\left(w^{-1} u\right)\right\rangle} d u
$$

(cf. [27], Prop. II.1.7). They satisfy functional equations:

$$
\begin{aligned}
& E_{P_{0}}^{G}(\lambda, \chi, g)=c(w, \lambda, \chi) E_{P_{0}}^{G}(w \lambda, w \chi, g) \\
& c\left(w^{\prime} w, \lambda, \chi\right)=c\left(w^{\prime}, w \lambda, w \chi\right) c(w, \lambda, \chi)
\end{aligned}
$$

(cf. [27], Théorème IV.1.10). Therefore, it suffices to calculate $c\left(w_{\alpha}, \lambda, \chi\right)$ for $\alpha \in \Delta_{0}\left(w_{\alpha}\right.$ corresponds to the reflection along $\alpha$ ).

Put $S_{\alpha}=\operatorname{Ker}(\alpha)^{0} \subset S_{0}$ and $G_{\alpha}=Z_{G}\left(S_{\alpha}\right)$. The Lie algebra of $G_{\alpha}$ is $\mathbf{a}_{0} \oplus \mathbf{g}_{-\alpha} \oplus \mathbf{g}_{\alpha}$. There is a homomorphism $\varphi_{\alpha}: S L_{2, F} \rightarrow \mathcal{D} G_{\alpha}$ (= derived group of $\left.G_{\alpha}\right)$ such that $d \varphi_{\alpha}$ maps the matrices

$$
\left(\begin{array}{ll}
0 & 1 \\
0 & 0
\end{array}\right),\left(\begin{array}{cc}
1 & 0 \\
0 & -1
\end{array}\right),\left(\begin{array}{ll}
0 & 0 \\
1 & 0
\end{array}\right)
$$

to $X_{\alpha}, H_{\alpha}, X_{-\alpha}$, respectively.

On $\mathbf{A}$ we take the measure $d x$ that is described in Tate's thesis (then $\operatorname{Vol}(F \backslash \mathbf{A})=1$ ). We have

$$
c\left(w_{\alpha}, \lambda, \chi\right)=\int_{\mathbf{A}} \phi_{\chi}\left(\varphi_{a}\left(\begin{array}{cc}
0 & -1 \\
1 & x
\end{array}\right)\right) \exp \left(\left\langle\lambda+\rho_{0}, H_{P_{0}}\left(\varphi_{\alpha}\left(\begin{array}{cc}
0 & -1 \\
1 & x
\end{array}\right)\right)\right\rangle\right) d x .
$$

It is an exercise to compute this integral. The result is

$$
c\left(w_{\alpha}, \lambda, \chi\right)=\frac{L(\chi \circ \check{\alpha},\langle\lambda, \alpha\rangle)}{L(\chi \circ \check{\alpha}, 1+\langle\lambda, \alpha\rangle)} .
$$


The Hecke $L$-functions are defined as follows. Let $\chi: \mathbf{G}_{m}(\mathbf{A}) / \mathbf{G}_{m}(F) \rightarrow S^{1}$ be an unramified character. For any finite place $v$ we put

$$
L_{v}\left(\chi_{v}, s\right)=\left(1-\chi_{v}\left(\pi_{v}\right)\left|\pi_{v}\right|_{v}^{s}\right)^{-1}
$$

and

$$
L_{f}(\chi, s)=\prod_{v \nmid \infty} L_{v}\left(\chi_{v}, s\right) .
$$

For any archimedean place $v$ there is a $\tau_{v} \in \mathbf{R}$ such that $\chi_{v}\left(x_{v}\right)=\left|x_{v}\right|_{v}^{i \tau_{v}}$ for all $x_{v} \in F_{v}^{*}$. Then

$$
L_{v}\left(\chi_{v}, s\right):=\left\{\begin{array}{clll}
\pi^{-\frac{\left(s+i \tau_{v}\right)}{2}} \Gamma\left(\frac{s+i \tau_{v}}{2}\right), & \text { if } & v & \text { is real } \\
(2 \pi)^{-\left(s+i \tau_{v}\right)} \Gamma\left(s+i \tau_{v}\right), & \text { if } & v & \text { is complex }
\end{array}\right.
$$

We define the complete Hecke $L$-function by

$$
L(\chi, s)=D^{s / 2} L_{\infty}(\chi, s) L_{f}(c, s),
$$

where $L_{\infty}(\chi, s)=\prod_{v \mid \infty} L_{v}\left(\chi_{v}, s\right)$ and $D=D(F / \mathbf{Q})$ is the absolute value of the discriminant of $F / \mathbf{Q}$. If the restriction of $\chi$ to $\mathbf{G}_{m}(\mathbf{A})^{1}$ is not trivial, then $L(\chi, s)$ is an entire function. If $\chi=1$, then $L(\chi, s)$ has exactly two poles of order one at $s=1$ and $s=0$. To state the functional equation, we let $\left(\pi_{v}^{d_{v}}\right)$ (with $d_{v} \geq 0$ and $d_{v}=0$ for almost all $v$ ) be the local discriminant of $F_{v}$ over the completion of $\mathbf{Q} \in F_{v}$ (for non-archimedean places $v)$. Put $\delta=\left(\delta_{v}\right)_{v} \in \mathbf{G}_{m}(\mathbf{A})$ with $\delta_{v}=1$ for all archimedean places and $\delta_{v}=\pi_{v}^{d_{v}}$ for all non-archimedean places. Then

$$
L(\chi, s)=\chi(\delta) L\left(\chi^{-1}, 1-s\right) .
$$

Using the functional equations (22) and (24) we get

$$
c(w, \lambda, \chi)=\prod_{\alpha>0, w \lambda<0} \frac{L(\chi \circ \check{\alpha},\langle\lambda, \alpha\rangle)}{L(\chi \circ \check{\alpha}, 1+\langle\lambda, \alpha\rangle)} .
$$

Proposition 8.3 Let $\Delta_{0}(\chi)$ be the set of $\alpha \in \Delta_{0}$ such that $\chi \circ \check{\alpha}=1$. Then

$$
\prod_{\alpha \in \Delta_{0}(\chi)}\langle\lambda, \alpha\rangle E_{P_{0}}^{G}\left(\lambda+\rho_{0}, \chi\right)
$$


has a holomorphic continuation to the tube domain over $-\frac{1}{4} \rho_{0}+X^{*}\left(P_{0}\right)^{+}$.

Proof. For $\alpha \in \Phi^{+}-\Delta_{0}$ we have $\left\langle\rho_{0}, \alpha\right\rangle \geq 2$ and therefore

$$
c\left(w_{\alpha}, \lambda+\rho_{0}, \chi\right)=\frac{L\left(\chi \circ \check{\alpha},\left\langle\lambda+\rho_{0}, \alpha\right\rangle\right)}{L\left(\chi \circ \check{\alpha}, 1+\left\langle\lambda+\rho_{0}, \alpha\right\rangle\right)}
$$

is holomorphic in this domain. If $\alpha \in \Delta_{0}-\Delta_{0}(\chi)$ then $\chi \circ \check{\alpha}$ restricted to $\mathbf{G}_{m}(\mathbf{A})^{1}$ is nontrivial, hence $c\left(w_{\alpha}, \lambda+\rho_{0}, \chi\right)$ is holomorphic in this domain too. For $\alpha \in \Delta_{0}(\chi)$ the function

$$
\langle\lambda, \alpha\rangle L\left(\chi \circ \check{\alpha},\left\langle\lambda+\rho_{0}, \alpha\right\rangle\right)=\langle\lambda, \alpha\rangle L(1,1+\langle\lambda, \alpha\rangle)
$$

is also holomorphic in this domain. This shows the holomorphy of

$$
\begin{gathered}
\prod_{\alpha \in \Delta_{0}(\chi)}\langle\lambda, \alpha\rangle E_{P_{0}}^{G}\left(\lambda+\rho_{0}, \chi\right)_{P_{0}}(g) \\
=\sum_{w \in \mathcal{W}} \prod_{\alpha \in \Delta_{0}(\chi)}\langle\lambda, \alpha\rangle c\left(w, \lambda+\rho_{0}, \chi\right) \phi_{w \chi}(g) e^{\left\langle w\left(\lambda+\rho_{0}\right)+\rho_{0}, H_{P_{0}}(g)\right\rangle}
\end{gathered}
$$

for $\operatorname{Re}(\lambda)$ contained in $-\frac{1}{4} \rho_{0}+X^{*}\left(P_{0}\right)^{+}$. By [27], Lemme I.4.10, we conclude that the same holds for $\prod_{\alpha \in \Delta_{0}(\chi)} E_{P_{0}}^{G}\left(\lambda+\rho_{0}, \chi\right)$.

8.4 Let $P=L U$ be a standard parabolic subgroup and $\chi \in \mathcal{U}_{P}$. For $\lambda \in X^{*}(P)_{\mathrm{C}}$ with $\operatorname{Re}(\lambda)$ contained in the interior of $X^{*}(P)^{+}$and $\vartheta$ contained in $X^{*}\left(P_{0}\right)^{+}$we have

$$
E_{P_{0}}^{G}\left(\vartheta+\lambda+\rho_{0}, \chi, g\right)=\sum_{\gamma \in P(F) \backslash G(F)} \phi_{\chi}(\gamma g) e^{\left\langle\lambda, H_{P}(\gamma g)\right\rangle} \sum_{\delta \in\left(L \cap P_{0}\right)(F) \backslash L(F)} e^{\left\langle\vartheta+2 \rho_{0}, H_{P_{0}}(\delta \gamma g)\right\rangle} .
$$

Let $w_{L}$ be the longest element of the Weyl group of $L$ (with respect to $S_{0}$ ) and define

$$
c_{P}:=\lim _{\vartheta \rightarrow \mathbf{0}, \vartheta \in X^{*}\left(P_{0}\right)^{+}}\left(\prod_{\alpha \in \Delta_{0}^{P}}\langle\vartheta, \alpha\rangle\right) c\left(w_{L}, \vartheta+\rho_{0}, 1\right) .
$$

By (26) this limit exists and is a positive real number.

Proposition 8.4 a)

$$
\lim _{\vartheta \rightarrow \mathbf{0}, \vartheta \in X^{*}\left(P_{0}\right)^{+}} \prod_{\alpha \in \Delta_{0}^{P}}\langle\vartheta, \alpha\rangle E_{P_{0}}^{G}\left(\vartheta+\lambda+\rho_{0}, \chi, g\right)=c_{P} E_{P}^{G}\left(\lambda+\rho_{P}, \chi, g\right) .
$$


b) Let $P^{\prime}=L^{\prime} U^{\prime}$ be a standard parabolic subgroup containing $P$ and suppose that $\chi \circ \check{\alpha}=1$ for all $\alpha \in \Delta_{0}^{P^{\prime}}$. Then $\chi \in \mathcal{U}_{P^{\prime}}$ and for all $\lambda \in X^{*}\left(P^{\prime}\right)_{\mathbf{C}}$ we have

$$
\lim _{\vartheta \rightarrow \mathbf{0}, \vartheta \in X^{*}(P)^{+}} \prod_{\alpha \in \Delta_{P}-\Delta_{P^{\prime}}}\langle\vartheta, \alpha\rangle E_{P}^{G}\left(\vartheta+\lambda+\rho_{P}, \chi, g\right)=\frac{c_{P^{\prime}}}{c_{P}} E_{P^{\prime}}^{G}\left(\lambda+\rho_{P^{\prime}}, \chi, g\right) .
$$

Proof. a) The proof rests on the fact that a measurable function of moderate growth on $L(F) \backslash L(\mathbf{A})$ for which all cuspidal components vanish vanishes almost everywhere. (For a proof cf. [27], Prop. I.3.4.) We claim that

$$
\lim _{\vartheta \rightarrow \mathbf{0}, \vartheta \in X^{*}\left(P_{0}\right)^{+}} \prod_{\alpha \in \Delta_{0}^{P}}\langle\vartheta, \alpha\rangle\left\{\sum_{\delta \in\left(L \cap P_{0}\right)(F) \backslash L(F)} e^{\left\langle\vartheta+2 \rho_{0}, H_{P_{0}}(\delta \gamma g)\right\rangle}\right\}=c_{P} e^{\left\langle 2 \rho_{P}, H_{P}(\gamma g)\right\rangle} .
$$

In fact, the cuspidal components of both sides along all non-minimal standard parabolic subgroups of $L$ vanish. To compare the constant terms along $P_{0} \cap L$ we can use (21) (for $L$ instead of $G$ and $P_{0} \cap L$ instead of $P_{0}$ ) and the explicit expression of the functions $c\left(w, \vartheta+\rho_{0}, 1\right)$ to get the identity stated above (note that $\left.w_{L} \rho_{0}+\rho_{0}=2 \rho_{P}\right)$.

b) Write $\chi=\chi_{0} \cdot \prod_{\alpha \in \Delta_{P}} \check{\alpha} \circ \varpi_{\alpha}$ with $\chi_{0} \in \mathcal{U}_{P^{\prime}}$. For $\alpha \in \Delta_{P}-\Delta_{P^{\prime}}$ we have $1=\chi \circ \check{\alpha}=$ $\chi_{0} \circ \check{\alpha}$. Thus $\chi=\chi_{0} \circ \prod_{\alpha \in \Delta_{P^{\prime}}} \check{\alpha} \circ \varpi_{\alpha} \in \mathcal{U}_{P^{\prime}}$. Using a) we get

$$
\begin{gathered}
\lim _{\vartheta \rightarrow \mathbf{0}, \vartheta \in X^{*}(P)^{+}} \prod_{\alpha \in \Delta_{P}-\Delta_{P^{\prime}}}\langle\vartheta, \alpha\rangle E_{P}^{G}\left(\vartheta+\lambda+\rho_{P}, \chi, g\right) \\
=\frac{1}{c_{P}} \lim _{\vartheta \rightarrow \mathbf{0}, \vartheta \in X^{*}(P)^{+}} \prod_{\alpha \in \Delta_{0}^{P^{\prime}}}\langle\vartheta, \alpha\rangle E_{P}^{G}\left(\vartheta+\lambda+\rho_{0}, \chi, g\right)=\frac{c_{P^{\prime}}}{c_{P}} E_{P^{\prime}}^{G}\left(\chi+\rho_{P^{\prime}}, \chi, g\right) .
\end{gathered}
$$

8.5 For $\chi \in\left(S_{0}(\mathbf{A}) / S_{0}(F)\left(S_{0}(\mathbf{A}) \cap \mathbf{K}_{G}\right)\right)^{*}$ and $v \in \mathrm{Val}_{\infty}(F)$ there is a character $\lambda_{v}=$ $\lambda_{v}(\chi) \in X^{*}\left(S_{0}\right)_{\mathbf{R}}$ such that for all $x \in S_{0}\left(F_{v}\right)$

$$
\chi(x)=e^{i \log \left(\left|\lambda_{v}(x)\right|_{v}\right)} .
$$

This gives a homomorphism

$$
\left(S_{0}(\mathbf{A}) / S_{0}(F)\left(S_{0}(\mathbf{A}) \cap \mathbf{K}_{G}\right)\right)^{*} \rightarrow X^{*}\left(S_{0}\right)_{\mathbf{R}}^{\mathrm{Val}_{\infty}(F)}
$$




$$
\lambda \mapsto \chi_{\infty}=\left(\lambda_{v}(\chi)\right)_{v \mid \infty}
$$

which has a finite kernel. The image of $\mathcal{U}_{0}$ under this map is a lattice of rank

$$
\left(\# \operatorname{Val}_{\infty}(F)-1\right) \operatorname{dim} S_{0} .
$$

Fix a norm $\|\cdot\|$ on $X^{*}\left(S_{0}\right)_{\mathbf{R}}$ and denote by the same symbol the induced maximum norm on $X^{*}\left(S_{0}\right)_{\mathbf{R}}^{\text {Val }_{\infty}(F)}$ (i.e., $\left.\left\|\left(\lambda_{v}\right)_{v \mid \infty}\right\|=\max _{v \mid \infty}\left\|l_{v}\right\|\right)$. Let $a, b>0$ and put

$$
B_{a, b}:=\left\{\lambda \in X^{*}\left(P_{0}\right)_{\mathbf{R}} \mid-\lambda+\frac{a}{2} \rho_{0} \in X^{*}\left(P_{0}\right)^{+}, \lambda+\frac{b}{2} \rho_{0} \in X^{*}\left(P_{0}\right)^{+}\right\} .
$$

This is a bounded convex open neighborhood of $\mathbf{0}$ in $X^{*}\left(P_{0}\right)_{\mathbf{R}}$. Note that if $\lambda \in B_{a, b}$, then $w_{0} \lambda+\frac{a}{2} \rho_{0} \in X^{*}\left(P_{0}\right)^{+}$, where $w_{0}$ is the longest element of $\mathcal{W}$.

Fix an $A>0$ such that

$$
\operatorname{Re}\left(\left\langle\lambda+s \rho_{0}, \alpha\right\rangle\left(\left\langle\lambda+s \rho_{0}, \alpha\right\rangle-1\right)\right)+A \geq 1
$$

for all $\lambda \in X^{*}\left(P_{0}\right)_{\mathrm{C}}$ with $\operatorname{Re}(\lambda) \in B_{a, b},-1-a \leq \operatorname{Re}(s) \leq 1+b$ and $\alpha \in \Phi^{+}$. Fix $\lambda \in X^{*}\left(P_{0}\right)_{\mathbf{C}}$ with $\operatorname{Re}(\lambda) \in B_{a, b}$ and $\chi \in \mathcal{U}_{0}$. Denote by $\Phi^{+}(\chi)$ the set of all positive roots $\alpha$ such that $\chi \circ \check{\alpha}=1$. Then

$$
\begin{gathered}
f_{\lambda, \chi}(s, g):= \\
\prod_{\alpha \in \Phi^{+}(\chi)} \frac{\left\langle\lambda+s \rho_{0}, \alpha\right\rangle\left(\left\langle\lambda+s \rho_{0}, \alpha\right\rangle-1\right)}{\left\langle\lambda+s \rho_{0}, \alpha\right\rangle\left(\left\langle\lambda+s \rho_{0}, \alpha\right\rangle-1\right)+A} \prod_{\alpha>0} L_{f}\left(\chi \circ \check{\alpha}, 1+\left\langle\lambda+s \rho_{0}, \alpha\right\rangle\right) E_{P_{0}}^{G}\left(\lambda+s \rho_{0}, \chi, g\right)
\end{gathered}
$$

is for any fixed $s$ in this strip an automorphic form on $G(F) \backslash G(\mathbf{A})$. Indeed, we observe that all cuspidal components of $f_{\lambda, c}(s, \cdot)$ along non-minimal standard parabolic subgroups vanish. Now we can use (21) and the explicit formulas for the functions $c(w, \lambda, \chi)$ in (25) to see that the constant term of $f_{\lambda, c}(s, \cdot)$ along $P_{0}$ is holomorphic for $s$ in this domain. By Lemme I.4.10 in [27] we can conclude that the same is true for $f_{\lambda, \chi}(s, \cdot)$.

It is our aim to apply a version of the Phragmén-Lindelöf principle due to Rademacher (cf. [34], Theorem 2) to the function

$$
s \mapsto f_{\lambda, \chi}(s, g)
$$

in the strip $-1-a \leq \operatorname{Re}(s) \leq 1+b$.

Using the functional equations of Eisenstein series (22) and $L$-functions (25) we get

$$
f_{\lambda, c}(-1-a-i t, g)=\prod_{\alpha \in \Phi^{+}(\chi)} \frac{\left\langle\lambda-(1+a+i t) \rho_{0}, \alpha\right\rangle\left(\left\langle\lambda-(1+a+i t) \rho_{0}, \alpha\right\rangle-1\right)}{\left\langle\lambda-(1+a+i t) \rho_{0}, \alpha\right\rangle\left(\left\langle\lambda-(1+a+i t) \rho_{0}, \alpha\right\rangle-1\right)+A}
$$




$$
\begin{aligned}
& \times \prod_{\alpha>0}(\chi \circ \check{\alpha})(\delta) D^{-\left\langle\lambda-(1+a+i t) \rho_{0}, \alpha\right\rangle} \prod_{\alpha>0} L_{f}\left(\left(w_{0} \chi\right) \circ \check{\alpha}, 1+\left\langle w_{0} \lambda+(1+a+i t) \rho_{0}, \alpha\right\rangle\right) \\
\times & \prod_{\alpha>0} \frac{L_{\infty}\left(\left(w_{0} \chi\right) \circ \check{\alpha}, 1+\left\langle w_{0} \lambda+(1+a+i t) \rho_{0}, \alpha\right\rangle\right)}{L_{\infty}\left(\left(w_{0} \chi\right)^{-1} \circ \check{\alpha}, 1-\left\langle w_{0} \lambda+(1+a+i t) \rho_{0}, \alpha\right\rangle\right)} E_{P_{0}}^{G}\left(w_{0} \lambda+(1+a+i t) \rho_{0}, w_{0} \chi, g\right) .
\end{aligned}
$$

Note that $L_{\infty}(\cdots)$ is a product of $\Gamma$-functions. Using the functional equation of the $\Gamma$ function we can derive the following estimate: There is $c>0$ depending only on $a$ and $b$ such that for $\operatorname{Re}(\lambda) \in B_{a, b}$ and $\chi \in \mathcal{U}_{0}$, we have

$$
\begin{gathered}
\left|f_{\lambda, \chi}(-1-a-i t, g)\right| \\
\leq c E_{P_{0}}^{G}\left(\operatorname{Re}\left(w_{0} \lambda\right)+(1+a) \rho_{0}, g\right) \times\left(1+\|\operatorname{Im}(\lambda)\|+\left\|\chi_{\infty}\right\|\right)^{\delta_{\mu}}|1+i t|^{\delta_{\mu}} .
\end{gathered}
$$

where $\delta_{\mu}:=\mu(2+a+b)$ and $\mu>0$ depends only on $F$ and $G$. Moreover, assuming $c$ to be big enough, we have also

$$
\left|f_{\lambda, \chi}(1+b+i t, g)\right| \leq c E_{P_{0}}^{G}\left(\operatorname{Re}(\lambda)+(1+b) \rho_{0}, g\right) .
$$

The proof of the following lemma was suggested to us by J. Franke.

Lemma 8.5 For $\operatorname{Re}(\lambda) \in B_{a, b}, \chi \in \mathcal{U}_{0}$ and $-1-a \leq \sigma \leq 1+b$ the following estimate holds:

$$
\begin{gathered}
\left|f_{\lambda, \chi}(\sigma+i t, g)\right| \leq c E_{P_{0}}^{G}\left(\operatorname{Re}(\lambda)+(1+b) \rho_{0}, g\right)^{\frac{\sigma+1+a}{2+a+b}} \\
\times\left\{E_{P_{0}}^{G}\left(\operatorname{Re}\left(w_{0} \lambda\right)+(1+a) \rho_{0}, g\right)\left(1+\|\operatorname{Im}(\lambda)\|+\left\|\chi_{\infty}\right\|\right)^{\delta_{\mu}}|2+a+\sigma+i t|^{\delta_{\mu}}\right\}^{\frac{1+b-\sigma}{2+a+b}} .
\end{gathered}
$$

Proof. This follows immediately from Theorem 2 in [34] once we have shown that for $-1-a \leq \sigma \leq 1+b$ we have

$$
\left|f_{\lambda, \chi}(\sigma+i t, g)\right| \leq c_{1} e^{|t|^{c_{2}}}
$$

for some $c_{1}, c_{2}>0$.

By (27) and (28), the function

$$
s \mapsto e^{s^{2}} f_{\lambda, \chi}(s, g)
$$

can be integrated over the lines $\operatorname{Re}(s)=-1-a-\epsilon$ and $\operatorname{Re}(s)=1+b+\epsilon$ for some $\epsilon>0$. We claim that for all $s$ with $-1-a \leq \operatorname{Re}(s) \leq 1+b$

$$
e^{s^{2}} f_{\lambda, \chi}(s, g)
$$




$$
=-\frac{1}{2 \pi i} \int_{\operatorname{Re}(z)=-1-a-\epsilon} \frac{e^{z^{2}} f_{\lambda, \chi}(z, g)}{z-s} d z+\frac{1}{2 \pi i} \int_{\operatorname{Re}(z)=1+b+c} \frac{e^{z^{2}} f_{\lambda, \chi}(z, g)}{z-s} d z .
$$

Denote the right-hand side by $h(s, g)$. This is a measurable function of moderate growth on $G(F) \backslash G(\mathbf{A})$ (cf. (27) and (28)). All cuspidal components of $h(s, \cdot)$ along non-minimal standard parabolic subgroups vanish. The same is true for the left-hand side.

It remains to compare the constant terms of both sides along $P_{0}$. By the absolute and uniform convergence of the integrals over the vertical lines, we see that the constant term of $h(s, \cdot)$ along $P_{0}$ is

$$
-\frac{1}{2 \pi i} \int_{\operatorname{Re}(z)=-1-a-\epsilon} \frac{g_{\lambda, \chi}(z, g)}{z-s} d z+\frac{1}{2 \pi i} \int_{\operatorname{Re}(z)=1+b+\epsilon} \frac{g_{\lambda, \chi}(z, g)}{z-s} d z .
$$

where

$$
g_{\lambda, \chi}(z, g):=\int_{U_{0}(F) \backslash U(\mathbf{A})} e^{z^{2}} f_{\lambda, \chi}(z, u g) d u .
$$

The explicit expression of the constant term of $E_{P_{0}}^{G}$ along $P_{0}$ in (21) and uniform estimates for $L$-functions as in [34], Theorem 5 (but for a larger strip), allow us to conclude that (31) is just the constant term of $e^{s^{2}} f_{\lambda, \chi}(s, \cdot)$ along $P_{0}$. Thus, by Proposition I.3.4 in [27], we have established (30). From (30) it follows that $\left|e^{s^{2}} f_{\lambda, \chi}(s, \cdot)\right|$ is bounded by some constant in the strip $-1-a \leq \operatorname{Re}(s) \leq 1+b$ and this in turn implies (29).

Proposition 8.6 Let $a>0$. For any $\epsilon>0$ there exist constants $b, c>0$ such that for all $\lambda \in X^{*}\left(P_{0}\right)_{\mathbf{C}}$ with $\operatorname{Re}(\lambda) \in B_{a, b}$ and $\chi \in \mathcal{U}_{0}$ we have

$$
\left|\prod_{\alpha \in \Delta_{0}(\chi)} \frac{\langle\lambda, \alpha\rangle}{\langle\lambda, \alpha\rangle+1} E_{P_{0}}^{G}\left(\lambda+\rho_{0}, \chi\right)\right| \leq c\left(1+\|\operatorname{Im}(\lambda)\|+\left\|\chi_{\infty}\right\|\right)^{\epsilon} .
$$

Proof. Note that if $\alpha \in \Phi^{+}-\Delta_{0}$ then $\left\langle\rho_{0}, \alpha\right\rangle \geq 2$ and hence $\left\langle\lambda+\rho_{0}, \alpha\right\rangle\left(\left\langle\lambda+\rho_{0}, \alpha\right\rangle-1\right)$ does not vanish for $\operatorname{Re}(\lambda) \in B_{a, b}$ and $b>0$ sufficiently small. For such $b$ there is a constant $c_{1}$ such that

$$
\left|\prod_{\alpha \in \Delta_{0}(\chi)} \frac{\langle\lambda, \alpha\rangle}{\langle\lambda, \alpha\rangle+1} E_{P_{0}}^{G}\left(\lambda+\rho_{0}, \chi\right)\right| \leq c\left|f_{\lambda, \chi}\left(1,1_{G}\right)\right| .
$$

Now we use the estimate for $\left|f_{\lambda, \chi}\left(1,1_{G}\right)\right|$ in Lemma 8.5 and require that $\mu b \leq \epsilon$. This gives the desired result. 
Proposition 8.7 Let $P$ be a standard parabolic subgroup of $G$. Let $a, \epsilon>0$. Then there exist $b, c>0$ such that for all $\chi \in \mathcal{U}_{P}$ and $\lambda \in X^{*}(P)_{\mathbf{C}}$ with $-\operatorname{Re}(\lambda)+\frac{a}{2} \rho_{0} \in X^{*}(P)^{+}$and $\operatorname{Re}(\lambda)+\frac{b}{2} \rho_{0} \in X^{*}(P)^{+}$we have

$$
\left|\prod_{\alpha \in \Delta_{P}(\chi)} \frac{\langle\lambda, \alpha\rangle}{\langle\lambda, \alpha\rangle+1} E_{P}^{G}\left(\lambda+\rho_{P}, \chi\right)\right| \leq c\left(1+\|\operatorname{Im}(\lambda)\|+\left\|\chi_{\infty}\right\|\right)^{\epsilon},
$$

where $\Delta_{P}(\chi)=\Delta_{0}(\chi) \cap \Delta_{P}$.

Proof. By the preceding proposition, there exist $b, c^{\prime}>0$ such that for all $\lambda^{\prime} \in X^{*}\left(P_{0}\right)_{\mathbf{C}}$ with $\operatorname{Re}\left(\lambda^{\prime}\right) \in B_{a, b}$ we have

$$
\left|\prod_{\alpha \in \Delta_{0}(\chi)} \frac{\left\langle\lambda^{\prime}, \alpha\right\rangle}{\left\langle\lambda^{\prime}, \alpha\right\rangle+1} E_{P_{0}}^{G}\left(\lambda^{\prime}+\rho_{0}, \chi\right)\right| \leq c^{\prime}\left(1+\left\|\operatorname{Im}\left(\lambda^{\prime}\right)\right\|+\left\|\chi_{\infty}\right\|\right)^{\epsilon} .
$$

Note that $\chi \circ \check{\alpha}=1$ for all $\alpha \in \Delta_{0}^{P}$ and hence $\Delta_{0}(\chi)=\Delta_{P}(\chi) \cup \Delta_{0}^{P}$. Now let $\lambda \in X^{*}(P)_{\mathbf{C}}$ be as in the proposition, i.e., $-\operatorname{Re}(\lambda)+\frac{a}{2} \rho_{P} \in X^{*}(P)^{+}$and $\operatorname{Re}(\lambda)+\frac{b}{2} \rho_{0} \in X^{*}(P)^{+}$. Then for all sufficiently small $\vartheta \in X^{*}\left(P_{0}\right)^{+}$we have

$$
\begin{gathered}
-(\vartheta+\operatorname{Re}(\lambda))+\frac{a}{2} \rho_{0} \in X^{*}\left(P_{0}\right)^{+} \\
\vartheta+\operatorname{Re}(\lambda)+\frac{b}{2} \rho_{0} \in X^{*}\left(P_{0}\right)^{+},
\end{gathered}
$$

i.e., $\vartheta+\operatorname{Re}(\lambda) \in B_{a, b}$. Hence for those $\vartheta$ we have

$$
\left|\prod_{\alpha \in \Delta_{0}(\chi)} \frac{\langle\vartheta+\lambda, \alpha\rangle}{\langle\vartheta+\lambda, \alpha\rangle+1} E_{P_{0}}^{G}\left(\vartheta+\lambda+\rho_{0}, \chi\right)\right| \leq c^{\prime}\left(1+\|\operatorname{Im}(\lambda)\|+\left\|\chi_{\infty}\right\|\right)^{\epsilon} .
$$

Letting $\vartheta$ tend to $\mathbf{0}$ and using Proposition 8.4 we can conclude that

$$
\left|\prod_{\alpha \in \Delta_{P}(\chi)} \frac{\langle\lambda, \alpha\rangle}{\langle\lambda, \alpha\rangle+1} E_{P}^{G}\left(\lambda+\rho_{P}, \chi\right)\right| \leq \frac{c^{\prime}}{c_{P}}\left(1+\|\operatorname{Im}(\lambda)\|+\left\|\chi_{\infty}\right\|\right)^{\epsilon} .
$$




\section{$9 \quad$ Examples}

This section is included to illustrate the foregoing constructions and techniques by means of two detailed examples which have been introduced in section 1.3.

9.1 The first example. For all $n \geq 1$ we had defined

$$
X_{n}:=\left\{\left(\left[y_{0}: y_{1}: y_{2}\right],\left[x_{0}: x_{1}\right]\right) \in \mathbf{P}^{2} \times \mathbf{P}^{1} \mid y_{0} x_{1}^{n}-y_{1} x_{0}^{n}=0\right\} .
$$

We consider $X_{n}$ as a variety over $F=\mathbf{Q}$. Let $T$ be the torus $\mathbf{G}_{m} \times \mathbf{G}_{m}$. Then

$$
M=X^{*}(T)=\mathbf{Z}_{\chi_{1}} \oplus \mathbf{Z}_{\chi_{2}},
$$

where $\chi_{i}$ is the projection of $T$ onto the $i$-th factor, and

$$
N=\operatorname{Hom}(M, \mathbf{Z})=\mathbf{Z} \delta_{1} \oplus \mathbf{Z} \delta_{2},
$$

where $\delta_{i}\left(\chi_{j}\right)=\delta_{i j}$. A fan $\Sigma$ is given as follows. The maximal cones are

$$
\begin{array}{ll}
\sigma_{1}=\mathbf{R}_{\geq 0} \delta_{2}+\mathbf{R}_{\geq 0} \delta_{1}, & \sigma_{2}=\mathbf{R}_{\geq 0} \delta_{1}+\mathbf{R}_{\geq 0}\left(-\delta_{2}\right), \\
\sigma_{3}=\mathbf{R}_{\geq 0}\left(-\delta_{2}\right)+\mathbf{R}_{\geq 0}\left(-\delta_{1}+n \delta_{2}\right), & \sigma_{4}=\mathbf{R}_{\geq 0}\left(-\delta_{1}+n \delta_{2}\right)+\mathbf{R}_{\geq 0} \delta_{2} .
\end{array}
$$

The fan $\Sigma$ consists of $\sigma_{1}, \ldots, \sigma_{4}$ and all cones obtained as intersections of these. The primitive integral generators of the one-dimensional cones in $\Sigma$ are

$$
e_{1}=\delta_{2}, e_{2}=\delta_{1}, e_{3}=-\delta_{2}, e_{4}=-\delta_{1}+n \delta_{2}
$$

The corresponding toric variety $X_{\Sigma}$ is isomorphic to $X_{n}$. To see this we cover both varieties with four $\mathbb{A}^{2}$ 's. For $X_{\Sigma}$ these $\mathbb{A}^{2}$ 's are given by the open subsets

$$
U_{\sigma_{i}}=\operatorname{Spec}\left(\mathbf{Q}\left[\sigma_{i}^{\vee} \cap M\right]\right), i=1, \ldots, 4 .
$$

The surface $X_{n}$ is covered by the four subsets

$$
\left\{x_{0} \neq 0, y_{2} \neq 0\right\},\left\{x_{0} \neq 0, y_{0} \neq 0\right\},\left\{x_{1} \neq 0, y_{2} \neq 0\right\},\left\{x_{1} \neq 0, y_{1} \neq 0\right\}
$$

Each of these open subsets is isomorphic to $\mathbb{A}^{2}$ and it is not difficult to write down the identifications of these affine pieces with the $U_{\sigma_{i}}$ above which patch together to give a global isomorphism (compare [15], 1.1). For instance, $U_{\sigma_{1}}=\operatorname{Spec}\left(\mathbf{Q}\left[\chi_{1}, \chi_{2}\right]\right)\left(\chi_{1}, \chi_{2}\right.$ 
generate the semigroup $\left.\sigma_{1}^{\vee} \cap M\right)$ and an isomorphism of $U_{\sigma_{1}}$ with the open subset $\left\{x_{0} \neq\right.$ $\left.0, y_{2} \neq 0\right\}$ is given by

$$
U_{\sigma_{1}} \ni\left(t_{1}, t_{2}\right) \mapsto\left(\left[t_{2}: t_{2} t_{1}^{n}: 1\right],\left[1: t_{1}\right]\right) \in X_{n}
$$

This isomorphism can be extended to an isomorphism from $X_{\Sigma}$ to $X_{n}$.

There is actually a second construction of $X_{n}$, namely as a twisted product. To realize $X_{n}$ in such a way we take $T=\mathbf{G}_{m}$ and let $X=\mathbf{P}^{1}$, viewed as an equivariant compactification of $T$. Put $G=S L_{2}$ and let $P$ be the Borel subgroup of upper triangular matrices in $G$. Define $\eta: P \rightarrow T$ by

$$
\left(\begin{array}{ll}
a & b \\
0 & d
\end{array}\right) \mapsto d^{n}
$$

Then $P$ acts on $X \times G$ from the right:

$$
(x, g) \cdot p=\left(x \eta(p), p^{-1} g\right) .
$$

The quotient $Y_{n}=(X \times G) / P$ is isomorphic to $X_{n}$. To see this we denote by $[[u: v], g]$ the point in $Y_{n}$ with representative $([u: v], g) \in X \times G$. Define

$$
Y_{n} \rightarrow X_{n}
$$

by

$$
\left[[u: v],\left(\begin{array}{cc}
a & b \\
c & d
\end{array}\right)\right] \mapsto\left(\left[u c^{n}: u d^{n}: v\right],[c: d]\right) .
$$

This is an isomorphism. Since this example is included to illustrate the "toric" constructions, we will henceforth consider $X_{n}$ as $X_{\Sigma}$ as above.

In the introduction we defined the height function induced by the Segre embedding of $X_{n}$ into $\mathbf{P}^{5}$ :

$$
\max \left\{\left|x_{0}\right|,\left|x_{1}\right|\right\} \cdot \max \left\{\left|y_{0}\right|,\left|y_{1}\right|,\left|y_{2}\right|\right\} \text {. }
$$

Now we will show how this height comes from a piecewise linear function on $N_{\mathbf{R}}$ as explained in section 3. Define

$$
\varphi_{0}: N_{\mathbf{R}}=\mathbf{R} \delta_{1} \oplus \mathbf{R} \delta_{2} \rightarrow \mathbf{R}
$$

by

$$
\varphi_{0}\left(x \delta_{1}+y \delta_{2}\right)=\max \{0, x\}+\max \{n \cdot \max \{0,-x\}, y\}
$$


One checks that $\varphi_{0}$ is a $\Sigma$-piecewise linear function on the space $N_{\mathbf{R}}$. For $\frac{a}{b}, \frac{c}{d} \in \mathbf{Q}^{*}$ with $\operatorname{gcd}(a, b)=\operatorname{gcd}(c, d)=1$ a straightforward calculation gives

$$
H_{\Sigma}\left(\left(\frac{a}{b}, \frac{c}{d}\right), \varphi_{0}\right)^{-1}=\max \{|a|,|b|\} \cdot \max \left\{|c| \cdot|a|^{n},|c| \cdot|b|^{n},|d| \cdot|b|^{n}\right\} .
$$

Therefore, under the isomorphism between $X_{\Sigma}$ and $X_{n}$ given above, this height function corresponds exactly to the height considered in the introduction.

9.2 Now we come to the counting of rational points by means of height zeta functions. Let us first consider the case when the points lie on the torus and denote by $X_{n}^{o} \subset X_{n}$ the corresponding open subset. Then then height zeta function is

$$
\sum_{\left(\left[y_{0}: y_{1}: y_{2}\right],\left[x_{0}: x_{1}\right]\right) \in X^{o}(\mathbf{Q})}\left(\max \left\{\left|x_{0}\right|,\left|x_{1}\right|\right\} \cdot \max \left\{\left|y_{0}\right|,\left|y_{1}\right|,\left|y_{2}\right|\right\}\right)^{-s} .
$$

Put $\varphi_{0}^{\prime}=\varphi_{0}-\frac{1}{2}\left(\chi_{1}+\chi_{2}\right)$. Then $\varphi_{0}^{\prime} \in P L(\Sigma)^{+}$and for $x \in T(\mathbf{Q})$ we have $H_{\Sigma}\left(x, \varphi_{0}^{\prime}\right)=$ $H_{\Sigma}\left(x, \varphi_{0}\right)$. By the preceding discussion we see that the height zeta function above is equal to

$$
Z(s):=\sum_{x \in T(\mathbf{Q})} H_{\Sigma}\left(x, s \varphi_{0}^{\prime}\right)
$$

and using Poisson's summation formula we get the following expression for this function:

$$
\frac{1}{\pi^{2}} \int_{M_{\mathbf{R}}} \hat{H}_{\Sigma}\left(1, s \varphi_{0}^{\prime}+i m\right) d m
$$

It is not difficult to calculate explicitely the Fourier transform $\hat{H}_{\Sigma}$ of the height function. Writing $m=\tau_{1} \chi_{1}+\tau_{2} \chi_{2} \in M_{\mathbf{R}}$ the result is

$$
\begin{gathered}
\hat{H}_{\Sigma}\left(1, s \varphi_{0}^{\prime}+i m\right)= \\
\times\left(\frac{\zeta\left(\frac{s}{2}+i \tau_{1}\right) \zeta\left(\frac{s}{2}+i \tau_{2}\right) \zeta\left(\frac{s}{2}-i \tau_{2}\right) \zeta\left(\frac{n+1}{2} s-i \tau_{1}+i n \tau_{2}\right)}{\zeta(s) \zeta\left(\frac{n+2}{2} s+i n \tau_{2}\right)}\right. \\
+\frac{1}{\left(\frac{s}{2}+i \tau_{2}\right)\left(\frac{s}{2}+i \tau_{1}\right)}+\frac{1}{\left(\frac{s}{2}+i \tau_{1}\right)\left(\frac{s}{2}-i \tau_{2}\right)}+\frac{1}{\left(\frac{s}{2}-i \tau_{2}\right)\left(\frac{n+1}{2} s-i \tau_{1}+i n \tau_{2}\right)} \\
\left.+\frac{1}{\left(\frac{n+1}{2} s-i \tau_{1}+i n \tau_{2}\right)\left(\frac{s}{2}+i \tau_{2}\right)}\right) .
\end{gathered}
$$


Next we will consider the cone of effective divisors inside the Picard group. Define a $\operatorname{map} \psi: P L(\Sigma) \rightarrow \mathbf{Z} \oplus \mathbf{Z} \simeq \operatorname{Pic}\left(X_{n}\right)$ by

$$
\varphi \mapsto\left(\varphi\left(\delta_{2}\right)+\varphi\left(-\delta_{2}\right), n \varphi\left(\delta_{2}\right)-\varphi\left(\delta_{1}\right)-\varphi\left(-\delta_{1}+n \delta_{2}\right)\right)
$$

The kernel of this map is precisely $M$ and hence it gives an identification if $\operatorname{Pic}\left(X_{n}\right)$ with $\mathbf{Z} \oplus \mathbf{Z}$. The cone of effective divisors $\Lambda_{\text {eff }}\left(X_{n}\right)$ in $\operatorname{Pic}\left(X_{n}\right)_{\mathbf{R}}$ is the image of $P L(\Sigma)^{+}$and is therefore generated by $\psi\left(\varphi_{1}\right)$ and $\psi\left(\varphi_{2}\right)\left(\varphi_{i}\left(e_{j}\right)=\delta_{i j}, 1 \leq i<j \leq 4\right)$. The element $\varphi_{\Sigma}=\varphi_{1}+\varphi_{2}+\varphi_{3}+\varphi_{4}$ is mapped to $(2, n-2)$ and $\psi\left(\varphi_{0}\right)=(1,-1)$.

Let $L$ be the line bundle corresponding to $\varphi_{0}$. Then $2 \psi\left(\varphi_{0}\right)-\psi\left(\varphi_{\Sigma}\right)=(0,-n)$ and thus $a(L)=2, b(L)=1$ (note that $n>0$ ). Moreover, $\Lambda(L)=\mathbf{R}_{\geq 0}(0,-1) \subset \Lambda_{\text {eff }}\left(X_{n}\right)$ and $\Sigma_{1}^{\prime}=\left\{e_{2}, e_{4}\right\}$. In the notations of section 5.4 we put $\varphi_{L}=\frac{n}{2} \varphi_{2}+\frac{n}{2} \varphi_{4}$. Then $\psi\left(\varphi_{0}\right)=\frac{1}{2}\left(\psi\left(\varphi_{\Sigma}+\varphi_{L}\right)\right)$ and we have $\hat{L}=\frac{1}{2}\left(\varphi_{\Sigma}+\varphi_{L}\right)$. By the definitions we made in section 5.5 we have:

$$
M^{\prime}=\mathbf{Z}_{\chi_{1}} \quad \text { and } \quad M^{\prime \prime}=M / M^{\prime} \simeq \mathbf{Z}_{\chi_{2}} .
$$

Moreover, $E=P L(\Sigma)_{\mathbf{R}} / M_{\mathbf{R}}^{\prime}$ and $V=M_{\mathbf{R}}^{\prime \prime}$. Put

$$
\begin{gathered}
h_{L}(\varphi)=\frac{\varphi\left(e_{1}\right)}{\varphi\left(e_{1}\right)+1} \cdot \frac{\varphi\left(e_{2}\right)}{\varphi\left(e_{2}\right)+1} \\
g(\varphi)=\frac{2}{\pi} \int_{M_{\mathbf{R}}^{\prime}} \hat{H}_{\Sigma}\left(1, \varphi+\varphi_{\Sigma}+\varphi_{L}+i m^{\prime}\right) h_{L}(\varphi) d m^{\prime} \\
f(\varphi)=g(\varphi) h_{L}(\varphi)^{-1} .
\end{gathered}
$$

Then

$$
Z(s+2)=\frac{1}{2 \pi} \int_{M_{\mathbf{R}}^{\prime \prime}} f\left(\frac{s}{2}\left(\varphi_{\Sigma}+\varphi_{L}\right)+i m^{\prime \prime}\right) d m^{\prime \prime} .
$$

By Theorem 5.6 we know that $Z(s)$ has a pole of order 1 in $s=2$ and a meromorphic continuation somewhat to the left of this pole. To compute the residue we recall the definitions of section 5.5. Put

$$
E^{(0)}=\operatorname{ker}\left(\varphi \mapsto \varphi\left(\delta_{2}\right)\right) \cap \operatorname{ker}\left(\varphi \mapsto \varphi\left(-\delta_{2}\right)\right) \subset E=P L(\Sigma)_{\mathbf{R}} / M_{\mathbf{R}}^{\prime}
$$

and let

$$
\pi_{0}: E \rightarrow E_{0}:=E / E^{(0)}
$$

be the canonical projection. Let

$$
E_{0}^{+}:=\left\{\bar{\varphi} \in E_{0} \mid \varphi\left(\delta_{2}\right) \geq 0, \varphi\left(-\delta_{2}\right) \geq 0\right\}
$$


$\psi_{0}: E_{0} \rightarrow P:=E_{0} / \pi_{0}\left(M_{\mathbf{R}}^{\prime \prime}\right)$ be the canonical projection and put $\Lambda:=\psi_{0}\left(E_{0}^{+}\right) \subset P$.

Define the $\mathcal{X}$-function $\mathcal{X}_{\Lambda}$ as in section 5.3. Then the residue of $Z(s)$ at $s=2$ is

$$
g(0) \mathcal{X}_{\Lambda}\left(\psi_{0}\left(\pi_{0}(\hat{L})\right)\right)
$$

We leave it to the reader to check that the second factor equals one. Let us compute $g(0)$ by the recipe given in section 6 . By Lemma 6.2 we have

$$
g(0)=2 \lim _{s \rightarrow 0} \prod_{e \in \Sigma_{1}-\Sigma_{1}^{\prime}}\left(s \varphi_{\Sigma}\right)(e) \sum_{x \in T^{\prime}(\mathbf{Q})} \int_{T^{\prime \prime}(\mathbf{A})} H_{\Sigma}\left(x t,(s+1) \varphi_{\Sigma}+\varphi_{L}\right) d t
$$

where $T^{\prime}=\operatorname{Spec}\left(\mathbf{Q}\left[M^{\prime}\right]\right), T^{\prime \prime}=\operatorname{Spec}\left(\mathbf{Q}\left[M^{\prime \prime}\right]\right)$.

Let $x=\frac{\alpha}{\beta} \in \mathbf{Q}^{*}, \operatorname{gcd}(\alpha, \beta)=1$. Then a computation similar to the one in section 4.4 gives

$$
\int_{T^{\prime \prime}(\mathbf{A})} H_{\Sigma}\left(x t,(s+1) \varphi_{\Sigma}+\varphi_{L}\right) d t=\frac{1}{\max \{|\alpha|, \beta \mid\}^{2\left(1+\frac{n}{2}+s\right)}} \cdot \frac{2}{1+s} \cdot \frac{\zeta(1+s)^{2}}{\zeta(2(1+s))} .
$$

Therefore,

$$
\begin{array}{r}
g(0)=2\left(\sum_{\frac{\alpha}{\beta} \in \mathbf{Q}^{*}} \frac{1}{\max \{|\alpha|, \beta \mid\}^{2\left(1+\frac{n}{2}\right)}}\right) \frac{2}{\zeta(2)} \\
=\frac{8}{\zeta(2) \zeta(n+2)}(2 \zeta(n+1)-\zeta(n+2)) .
\end{array}
$$

Now let us look at those rational points on $X_{n}$ which do not lie on the torus. They are on the four $\mathbf{P}^{1}$ s:

$$
\left\{x_{0}=y_{0}=0\right\},\left\{x_{1}=y_{1}=0\right\},\left\{y_{0}=y_{1}=0\right\},\left\{y_{2}=0\right\} .
$$

Only the rational points on the first three curves contribute to the asymptotic behavior of the counting function and each time the zeta function has a simple pole at $s=2$ with residue $\frac{4}{\zeta(2)}$. Collecting all terms together and dividing by 2 (remember the formula for the asymptotic constant in section 1.4) we get

$$
N(H) \sim \frac{8 \zeta(n+1)+2 \zeta(n+2)}{\zeta(2) \zeta(n+2)} H^{2} .
$$


9.3 The second example. Here we will present a really mixed case, i.e., a toric bundle over a flag variety which is neither a toric variety nor a homogeneous space.

Let us recall the construction of this variety (cf. section 1.3). We introduce homogeneous coordinates $x_{0}, x_{i j}, x_{i j}^{\prime}, 1 \leq i<j \leq 4$ in $\mathbf{P}^{12}$ and homogeneous coordinates $w_{i j}, 1 \leq i<j \leq 4$ in $\mathbf{P}^{5}$. The subvariety $Y$ of $\mathbf{P}^{12} \times \mathbf{P}^{5}$ is defined by the equations:

$$
\begin{gathered}
w_{12} w_{34}-w_{13} w_{24}+w_{14} w_{23}=0 \\
\text { for all } 1 \leq i<j \leq 4,1 \leq k<l \leq 4 \quad \begin{array}{l}
x_{i j} w_{k l}-x_{k l} w_{i j}=0, \\
\\
x_{i j}^{\prime} w_{k l}^{2}-x_{k l}^{\prime} w_{i j}^{2}=0 .
\end{array}
\end{gathered}
$$

For a Q-rational point on $Y$ represented by primitive integral vectors $\left(x_{0}, x_{i j}, x_{i j}^{\prime}\right),\left(w_{i j}\right)$ we considered the height

$$
\max \left\{\left|x_{0}\right|,\left(\sum x_{i j}^{2}\right)^{1 / 2}, \sum\left|x_{i j}^{\prime}\right|\right\}^{3} \cdot\left(\sum w_{i j}^{2}\right)^{1 / 2} .
$$

The reader may have noticed that $Y$ is a $\mathbf{P}^{2}$-bundle over the Grassmannian of 2-planes in a four-dimensional vector space. To see this, let $G=S L_{4}$ and $P=\left\{\left(\begin{array}{cc}a & b \\ 0 & d\end{array}\right)\right\} \subset G$, where $a, b, d$ are $2 \times 2$-matrices. Then $P \backslash G$ is the Grassmannian $G r(2,4)$ and the Plücker embedding $P \backslash G \hookrightarrow \mathbf{P}^{5}$ can be described as follows: Write $g=\left(a_{\alpha \beta}\right)_{1 \leq \alpha, \beta \leq 4} \in G$. Then $P g \in P \backslash G$ is mapped to

$$
\left[w_{12}: w_{13}: w_{14}: w_{23}: w_{24}: w_{34}\right] \in \mathbf{P}^{5}
$$

with $w_{i j}=a_{3 i} a_{4 j}-a_{3 j} a_{4 i}$. The image is the hypersurface

$$
w_{12} w_{34}-w_{13} w_{24}+w_{14} w_{23}=0 .
$$

Let $T=\mathbf{G}_{m} \times \mathbf{G}_{m}, M=\mathbf{Z} \chi_{1} \oplus \mathbf{Z} \chi_{2}, N=\mathbf{Z} \delta_{1} \oplus \mathbf{Z} \delta_{2}$ with the same conventions as in the first example. Let $\Sigma$ be the fan in $N_{\mathbf{R}}$ with the following maximal cones:

$$
\sigma_{1}=\mathbf{R}_{\geq 0} \delta_{2}+\mathbf{R}_{\geq 0} \delta_{1}, \sigma_{2}=\mathbf{R}_{\geq 0} \delta_{2}+\mathbf{R}_{\geq 0}\left(-\delta_{1}-\delta_{2}\right), \sigma_{3}=\mathbf{R}_{\geq 0}\left(-\delta_{1}-\delta_{2}\right)+\mathbf{R}_{\geq 0} \delta_{2} .
$$

Then $X=X_{\Sigma}$ is isomorphic to $\mathbf{P}^{2}$ and we may embed $T \hookrightarrow \mathbf{P}^{2}$ by

$$
\left(t_{1}, t_{2}\right) \mapsto\left[1: t_{1}: t_{2}\right]
$$

Define $\eta: P \rightarrow T$ by

$$
p=\left(\begin{array}{ll}
a & b \\
0 & d
\end{array}\right) \mapsto\left(\operatorname{det}(d), \operatorname{det}(d)^{2}\right)
$$


Then $P$ acts on $X \times G$ from the right:

$$
(x, g) \cdot p=\left(x \eta(p), p^{-1} g\right)
$$

and the quotient $(X \times G) / P$ will be identified with $Y$ by means of the following isomorphism:

$$
\left.\left(\left[x_{0}: x_{1}: x_{2}\right], g=\left(a_{\alpha, \beta}\right)\right) P \mapsto\left(x_{0}: x_{i j}=x_{1} w_{i j}: x_{i j}^{\prime}=x_{2} w_{i j}^{2}\right],\left[w_{i j}\right]\right),
$$

where $w_{i}=a_{3 i} a_{4 j}-a_{3 j} a_{4 i}$. Next we will interpret the height function given above in terms used in sections 2-4. The $\Sigma$-piecewise linear function $\varphi_{\Sigma}$ is given by

$$
\varphi_{\Sigma}\left(x \delta_{1}+y \delta_{2}\right)=\max \{x+y, y-2 x, x-2 y\} .
$$

In section 4.3 we introduced the metrized line bundle $\mathcal{L}_{\varphi_{\Sigma}}^{Y}$ on $Y$. For this purpose we chose a maximal compact subgroup $\mathbf{K}=\prod_{v} \mathbf{K}_{v} \subset G(\mathbf{A})$. Here we put $\mathbf{K}_{v}=S L_{4}\left(\mathbf{Z}_{p}\right)$ for $v=p$ and $\mathbf{K}_{v_{\infty}}=S O_{4}(\mathbf{R})$. Define $Y^{o}=(T \times G) / P$ and let $y=(x, \gamma) P \in Y^{o}(\mathbf{Q})$. We may assume $\gamma=\left(a_{\alpha \beta}\right)_{1 \leq \alpha, \beta \leq 4} \in S L_{4}(\mathbf{Z})$. For finite $v\left(=v_{p}\right)$ let $p_{v}=1$ and $k_{v}=\gamma \in \mathbf{K}_{v}$. Write $\gamma=p_{\gamma} k_{\gamma}$ with $p_{\gamma}=\left(p_{v}\right)_{v}$ and $k_{\gamma}=\left(k_{v}\right)_{v}$. Then

$$
\eta\left(p_{\gamma}\right)=\left( \pm\left(\sum w_{i j}^{2}\right)^{1 / 2}, \sum w_{i j}^{2}\right)
$$

where $w_{i j}=a_{3 i} a_{4 j}-a_{3 j} a_{4 i}$. Let $x=\left(\frac{b}{a}, \frac{c}{a}\right) \in T(\mathbf{Q}), \operatorname{gcd}(a, b, c)=1$. Then

$$
\begin{gathered}
H_{\mathcal{L}_{\varphi_{\Sigma}}^{Y}}(y)=H_{\Sigma}\left(x \eta\left(p_{\gamma}\right),-\varphi_{\Sigma}\right) \\
=\max \left\{|a|\left(\sum w_{i j}^{2}\right)^{-1 / 2},|b|,|c|\left(\sum w_{i j}^{2}\right)^{1 / 2}\right\}^{3} .
\end{gathered}
$$

Define $\lambda_{0}: P \rightarrow \mathbf{G}_{m}$ by $\lambda_{0}\left(\begin{array}{ll}a & b \\ 0 & d\end{array}\right)=\operatorname{det}(d)^{-1}$. Then $2 \rho_{P}$ (cf. section 2.1) is $4 \lambda_{0}$ and

$$
H_{\pi^{*} \mathcal{L}_{2 \rho_{P}}}(y)=H_{\mathcal{L}_{2 \rho_{P}}}(\gamma P)=\left|\lambda_{0}\left(p_{\gamma}\right)\right|^{4}=\left(\sum w_{i j}^{2}\right)^{2} .
$$

Thus we see that

$$
\begin{aligned}
H_{\mathcal{L}_{\varphi_{\Sigma}}^{Y} \otimes \pi^{*} \mathcal{L}_{2 \rho_{P}}}(y) & =\left(\sum w_{i j}^{2}\right)^{1 / 2} \max \left\{|a|,|b|\left(\sum w_{i j}^{2}\right)^{1 / 2},|c|\left(\sum w_{i j}^{2}\right)\right\}^{3} \\
& =\left(\sum w_{i j}^{2}\right)^{1 / 2} \max \left\{|a|,\left(\sum\left(b w_{i j}\right)^{2}\right)^{1 / 2}, \sum\left|c w_{i j}^{2}\right|\right\}^{3} .
\end{aligned}
$$

Hence, under the isomorphism described above, the height considered before is exactly the height function for the metrized line bundle $\mathcal{L}_{\varphi_{\Sigma}}^{Y} \otimes \pi^{*} \mathcal{L}_{2 \rho_{P}}$ and this is a metrization of the anticanonical line bundle $L=L_{\varphi_{\Sigma}}^{Y} \otimes \pi^{*} L_{2 \rho_{P}}$. 
9.4 Next we will consider the corresponding height zeta function, i.e., the height zeta function for $Y^{o}$ and for the height introduced above. If we view $Y$ as a subvariety of $\mathbf{P}^{12} \times \mathbf{P}^{5}$, the zeta function takes the form

$$
\sum \frac{1}{\left(\left(\sum w_{i j}^{2}\right)^{1 / 2} \max \left\{\left|x_{0}\right|,\left(\sum x_{i j}^{2}\right)^{1 / 2}, \sum\left|x_{i j}^{\prime}\right|\right\}\right)^{s}},
$$

where the summation is taken over all Q-rational points on $Y^{o}$ represented by primitive integral vectors $\left(x_{0}, x_{i j}, x_{i j}^{\prime}\right)$ and $\left(w_{i j}\right)$. On the other hand, by the preceding discussion, this is equal to

$$
Z(s):=\sum_{y \in Y^{o}(\mathbf{Q})} H_{\mathcal{L}_{\varphi_{\Sigma}}^{Y} \otimes \pi^{*} \mathcal{L}_{2 \rho_{P}}}(y)^{-s} .
$$

In Proposition 4.4 we obtained another expression for this function, namely

$$
\frac{1}{\pi^{2}} \int_{M_{\mathbf{R}}} \hat{H}_{\Sigma}\left(1, s \varphi_{\Sigma}+i m\right) E_{P}^{G}\left(s 2 \rho_{P}-\rho_{P}-i \check{\eta}(m), 1\right) d m .
$$

Writing $m=\tau_{1} \chi_{1}+\tau_{2} \chi_{2} \in M_{\mathbf{R}}$ we have

$$
\hat{H}_{\Sigma}\left(1, s \varphi_{\Sigma}+i m\right)=\frac{3 s}{\zeta(3 s)} \frac{\zeta\left(s+i \tau_{1}\right) \zeta\left(s+i \tau_{2}\right) \zeta\left(s-i\left(\tau_{1}+\tau_{2}\right)\right)}{\left(s+i \tau_{1}\right)\left(s+i \tau_{2}\right)\left(s-i\left(\tau_{1}+\tau_{2}\right)\right)}
$$

Further, $\check{\eta}(m)=-\tau_{1} \lambda_{0}-2 \tau_{2} \lambda_{0}$ and

$$
E_{P}^{G}\left((2 s-1) \rho_{P}-i \check{\eta}(m)\right)=\sum_{\gamma \in P(\mathbf{Q}) \backslash G(\mathbf{Q})}\left(\sum w_{i j}^{2}\right)^{-\frac{1}{2}\left(4 s-i \tau_{1}-2 i \tau_{2}\right)},
$$

with $\gamma$ again represented by $\left(a_{\alpha \beta}\right)_{1 \leq \alpha, \beta \leq 4} \in S L_{4}(\mathbf{Z})$ and $w_{i j}=a_{3 i} a_{4 j}-a_{3 j} a_{4 i}$. The abscissa of convergence lies at $a(L)$ which is equal to one. Define $h_{L}$ as in section 5.4 and put

$$
\begin{gathered}
g(\varphi, \lambda)=4 \hat{H}_{\Sigma}\left(1, \varphi+\varphi_{\Sigma}\right) E_{P}^{G}\left(\lambda+\rho_{P}, 1\right) h_{L}(\varphi, \lambda) \\
f(\varphi, \lambda)=g(\varphi, \lambda) h_{L}(\varphi, \lambda)^{-1}, \hat{L}=\left(\varphi_{\Sigma}, 2 \rho_{P}\right) \in P L(\Sigma) \oplus X^{*}(P) .
\end{gathered}
$$

Then

$$
Z(s+1)=\frac{1}{(2 \pi)^{2}} \int_{M_{\mathbf{R}}} f\left(s\left(\varphi_{\Sigma}, 2 \rho_{P}\right)+i(m,-\check{\eta}(m))\right) d m .
$$

By Theorem 5.6 this function has a pole of order 2 at $s=1$ with leading coefficient

$$
g(0) \mathcal{X}_{\Lambda_{\text {eff }}(Y)}\left(\psi\left(\varphi_{\Sigma}, 2 \rho_{P}\right)\right)
$$


where $\psi: P L(\Sigma) \oplus X^{*}(P) \rightarrow \operatorname{Pic}(Y) \simeq \mathbf{Z} \oplus \mathbf{Z}$ may be described by

$$
\left(\varphi, a \lambda_{0}\right) \mapsto\left(\varphi\left(\delta_{2}\right)+\varphi\left(\delta_{1}\right)+\varphi\left(-\delta_{1}-\delta_{2}\right), 2 \varphi\left(\delta_{2}\right)+\varphi\left(\delta_{1}\right)-a\right) .
$$

The effective cone is the image of $P L(\Sigma)^{+} \times X^{*}(P)$ and is therefore spanned by $(1,2)$ and $(0,-1)$. Now one can compute the value of the $\mathcal{X}$-function at $\psi\left(\varphi_{\Sigma}, 2 \rho_{P}\right)=(3,-1)\left(2 \rho_{p}=\right.$ $\left.4 \lambda_{0}\right)$ :

$$
\mathcal{X}_{\Lambda_{\mathrm{eff}}(Y)}\left(\psi\left(\varphi_{\Sigma}, 2 \rho_{P}\right)\right)=\frac{1}{21}
$$

The constant $g(0)$ has a part coming from $\hat{H}_{\Sigma}$ which is equal to $\frac{3}{\zeta(3)}$ and a part coming from the Eisenstein series, which is

$$
\frac{c_{G}}{c_{P}}=\pi^{2} \frac{\zeta(2)}{\zeta(3) \zeta(4)}
$$

(in the notation of Lemma 6.1, cf. 8.4 for the definitions).

Collecting all terms together we get a closed formula for the leading coefficient of the height zeta function at $s=1$. Thus (cf. 1.4 for the connection of the leading coefficient and the asymptotic constant) the number of rational points on $Y^{o}$ with height bounded by $H$ is asymptotically equal to

$$
\frac{4}{7} \pi^{2} \frac{\zeta(2)}{\zeta(3)^{2} \zeta(4)} H \cdot \log H .
$$

\section{References}

[1] S. J. Arakelov, Theory of intersections on the arithmetic surface, Proc. of Int. Congress of Math. Vol. 1, Montreal 1975, 405-408.

[2] V. Batyrev, Yu. I. Manin, Sur le nombre des points rationnels de hauteur bornée des variétés algebriques, Math. Ann. 286 (1990), 27-43.

[3] V. Batyrev, Yu. Tschinkel, Rational points of bounded height on compactifications of anisotropic tori, Intern. Math. Research Notices 12 (1995), 591-635.

[4] V. Batyrev, Yu. Tschinkel, Manin's conjecture for toric varieties, J. Alg. Geom. 7(1) (1999), 15-53.

[5] V. Batyrev, Yu. Tschinkel, Height zeta functions of toric varieties, Algebraic geometry 5, (Manin's Festschrift), J. Math. Sciences 82(1) (1996), 3220-3239. 
[6] V. Batyrev, Yu. Tschinkel, Rational points on some Fano cubic bundles, C.R. Acad. Sci., Paris 323 Ser. I (1996), 41-46.

[7] V. Batyrev, Yu. Tschinkel, Tamagawa numbers of polarized algebraic varieties, Astérisque 251 1998, 299-340.

[8] B. J. Birch, Forms in many variables, Proc. Roy. Soc. Ser. A 265 (1961/1962), 245263.

[9] R. de la Bretéche, Sur la hauteur des points d'une certaine surface cubique singulière, Astérisque 251 1998, 51-77.

[10] F. Bruhat, p-adic groups, in: Algebraic groups and Discontinuous subgroups, Proc. of Symp. in Pure Math. 9 (ed. by A. Borel, G. Mostow), AMS, Providence, Rhode Island 1966, 63-70.

[11] H. Delange, Généralisation du théorème de Ikehara, Ann. Sci. ENS 71 (1954), 213242.

[12] G. Faltings, Calculus on arithmetic surfaces, Ann. of Math. 119(2) (1984), 387-424.

[13] E. Fouvry, Sur la hauteur de points d'une certain surface cubique singulière, Astérisque 51 1998, 31-49.

[14] J. Franke, Yu.I. Manin, Yu. Tschinkel, Rational points of bounded height on Fano varieties, Inv. Math. 95 (1989), 421-435.

[15] W. Fulton, Introduction to Toric Varieties, Annals of Mathematics Studies 131, Princeton University Press, Princeton, NJ, 1993.

[16] R. Godement, Introduction à la théorie de Langlands, Sém. Bourbaki 321 (1966/67).

[17] R. Hartshorne, Algebraic Geometry, Springer, New York, 1977.

[18] L. Hörmander, An Introduction to Complex Analysis in Several Variables, 3rd. edition, North-Holland, Amsterdam, 1990.

[19] J. C. Jantzen, Representations of Algebraic Groups, Academic Press, Orlando, Florida, 1987.

[20] G. Kempf, F. Knudsen, D. Mumford, B. Saint-Donat, Toroidal Embeddings I, LNM 339, Springer, Berlin, 1973. 
[21] S. Lang, Diophantine Geometry, Interscience Tracts in Pure and Applied Mathematics, 11, Interscience Publishers, New York-London, 1962.

[22] S. Lang, Fundamentals of Diophantine Geometry, Springer-Verlag, New York-Berlin, 1983.

[23] S. Lang, Number theory III - Diophantine Geometry, Encyclopaedia of Mathematical Sciences 60, Springer-Verlag, Berlin, 1991.

[24] Yu. I. Manin, Notes on the arithmetic of Fano threefolds, Compositio Math. 85(1) (1993), 37-55.

[25] J. G. M. Mars, Sur l'approximation du nombre de solutions de certaines equations diophantiennes, Ann. Sci. ENS (4) 6 (1973), 357-387.

[26] B. Z. Moroz, On the integer points of some toric varieties, Quart. J. Math., Oxford Ser. (2) 48, 189 (1997), 67-82.

[27] C. Moeglin, J.-L. Waldspurger, Décomposition Spectrale et Séries d'Eisenstein. Une Paraphrase de l'Écriture, Progress in Mathematics 113, Birkhäuser Basel, 1994.

[28] D. Mumford, J. Fogarty, F. Kirwan, Geometric Invariant Theory, 3rd edition, Springer, Berlin, 1994.

[29] A. Néron, Quasi-fonctions et hauteurs sur les variétés abéliennes, Ann. of Math. (2) 82 (1965), 249-331.

[30] T. Oda, Convex Bodies and Algebraic Geometry. An Introduction to the Theory of Toric Varieties, Ergebnisse der Mathematik und ihrer Grenzgebiete. 3. Folge, Bd. 15, Springer, Berlin, 1988.

[31] E. Peyre, Hauteurs et nombres de Tamagawa sur les variétés de Fano, Duke Math. J. 79 (1995), 101-218.

[32] E. Peyre, Terme principal de la fonction zeta des hauteurs et torseurs universels, Astérisque 251 (1998), 259-298.

[33] E. Peyre, Torseurs universels et méthode du cercle, preprint (1998).

[34] H. Rademacher, On the Phragmén-Lindelöf theorem and some applications, Math. Zeitschrift 72 (1959), 192-204. 
[35] M. Reid, Decomposition of toric morphisms, in: Arithmetic and Geometry, Vol. II: Geometry, Prog. in Math. 36, Birkhäuser, Basel (1983), 395-418.

[36] P. Salberger, Tamagawa measures on universal torsors and points of bounded height on Fano varieties, Astérisque 251 (1998), 91-258.

[37] J.-J. Sansuc, Groupe de Brauer et arithmétique des groupes algébriques linéaires sur un corps de nombres, Journal für die reine und angewandte Math. 327 (1981), 12-80.

[38] S. H. Schanuel, On heights in number fields, Bull. Amer. Math. Soc. 70 (1964), $262-263$.

[39] S. H. Schanuel, Heights in number fields, Bull. Soc. Math. France 107(4) (1979), 433-449.

[40] W. Schmidt, The density of integer points on homogeneous varieties, Acta Math. 154 no. 3-4 (1985), 243-296.

[41] W. Schmidt, Asymptotic formulae for point lattices of bounded determinant and subspaces of bounded height, Duke Math. J. 35 (1968), 327-339.

[42] M.A. Shubin, Pseudodifferential Operators and Spectral Theory, Springer, Berlin, 1987.

[43] J. Silverman, The theory of height functions, in Arithmetic geometry, Springer, New York, 1986, 151-166.

[44] M. Strauch, Höhentheoretische Zetafunktionen von Faserbündeln über verallgemeinerten Fahnenvarietäten, Ph.D. Thesis, Universität Bonn, (1998), to appear in Bonner Mathematische Schriften.

[45] M. Strauch, Yu. Tschinkel, Height zeta functions of twisted products, Math. Res. Letters 4 (1997), 273-282.

[46] J. Tate, Fourier analysis in number fields and Hecke's zeta-functions, in Algebraic Number Theory, ed. J.W.S. Cassels, A. Fröhlich, Acad. Press, 1967.

Matthias Strauch

Mathematisches Institut

Westfälische Wilhelms-Universität Münster

Einsteinstrasse 62, 48149 Münster, Germany 
e-mail: straucm@math.uni-muenster.de

and

Yuri Tschinkel

Dept. of Mathematics, U.I.C.

Chicago, (IL) 60607-7045, U.S.A.

e-mail: yuri@math.uic.edu 